\author{
Universidade de São Paulo \\ Instituto de Física
}

\title{
Transporte, escape de partículas e propriedades dinâmicas de mapeamentos não lineares
}

\author{
Diogo Ricardo da Costa
}

Orientador: Prof. Dr. Edson Denis Leonel

Co-Orientador: Prof. Dr. Iberê Luiz Caldas

Tese de doutorado apresentada ao Instituto de Física

para a obtenção do título de Doutor em Ciências

Banca Examinadora:

Prof. Dr. Edson Denis Leonel (IFUSP)

Prof. Dr. José Carlos Sartorelli (IFUSP)

Prof. Dr. José Roberto Ruggiero (UNESP - Rio Preto)

Prof. Dr. Paulo César Rech (UDESC)

Prof. Dr. Marcus Beims (UFPR) 


\section{FICHA CATALOGRÁFICA}

\section{Preparada pelo Serviço de Biblioteca e Informação}

do Instituto de Física da Universidade de São Paulo

Costa, Diogo Ricardo da

Transporte, escape de partículas e propriedades dinâmicas de mapeamentos não lineares. São Paulo, 2014.

Tese (Doutorado) - Universidade de São Paulo. Instituto de Física - Depto. de Física Aplicada

Orientador: Prof. Dr. Edson Denis Leonel

Área de Concentração: Física Aplicada

Unitermos: 1. Mecânica Estatística Clássica; 2. Sistemas

Dinâmicos; 3. Caos (Sistemas Dinâmicos).

USP/IF/SBI-016/2014 


\section{Agradecimentos}

Ao meu orientador Prof. Dr. Edson Denis Leonel e co-orientador Prof. Dr. Iberê Luiz Caldas, pela paciência, esforço e dedicação durante o doutorado.

Aos pesquisadores do Grupo de Estudos de Sistemas Complexos e Dinâmica Não Linear da UNESP de Rio Claro, que possui como líder o Prof. Dr. Edson Denis Leonel.

Ao DEMAC (Departamento de Estatística, Matemática Aplicada e Computação) e departamento de Física da UNESP e Rio Claro por disponibilizar o laboratório e acesso computacional. Esta pesquisa tornou-se possível graças aos recursos computacionais disponibilizados pelo Núcleo de Computação Científica (NCC/GridUNESP) da Universidade Estadual Paulista (UNESP) e ao cluster computacional do grupo de Estudos de Sistemas Complexos e Dinâmica Não Linear, adquirido pelos projetos FAPESP 2012/23688-5, 2008/57528-9 e 2005/56253-8.

À todos os Profs. das disciplinas de Pós-Graduação que cursei ao longo do doutoramento.

Aos pesquisadores Mário R. Silva, Carl P. Dettmann, Juliano A. de Oliveira e Diego F. M. de Oliveira, com o qual tive oportunidade de publicar e submeter alguns trabalhos.

Aos colegas de Pós-Graduação, que de alguma forma colaboraram no desenvolvimento deste trabalho.

À CAPES, CNPq e FAPESP (2010/52709-5) pelo apoio financeiro durante a realização deste projeto.

Ao grupo do prof. Carl P. Dettmann (Bristol - Inglaterra) pela visita de 01/02/2013 à 31/07/2013 com bolsa BEPE - FAPESP de doutorado sanduíche (2012/18962-0).

Aos demais funcionários e docentes da Universidade de São Paulo.

Muito obrigado!

Diogo Ricardo da Costa 


\section{Resumo}

Investigaremos algumas propriedades dinâmicas e de transporte para um conjunto de partículas clássicas não interagentes em diversos sistemas físicos. Os sistemas descritos aqui, em sua maioria, apresentam estrutura mista no espaço de fase no sentido de que curvas invariantes do tipo spanning, mares de caos e ilhas periódicas estão presentes. A descrição de cada sistema será feita utilizando mapeamentos discretos não lineares. Detalharemos a forma de obter os mapeamentos assim como discutiremos algumas de suas propriedades dinâmicas. Expoentes de Lyapunov serão utilizados para caracterizar a região de caos nos sistemas. Hipóteses de escala são usadas para provar que certos observáveis, por exemplo a energia média ao longo de mares de caos, são invariantes de escala. Consideraremos também que quando uma partícula, ou de forma equivalente um conjunto delas atinge uma determinada altura no espaço de fases, ela pode escapar. Ao estudar o escape de partículas, vemos que o histograma do número de partículas que atingem uma certa altura (ou energia) $h$ no espaço de fases em uma dada iterada $n$, ao qual observamos ser invariante de escala, cresce rapidamente até atingir um máximo e então tende à zero para $n$ grande. Quando a altura $h$ varia proporcionalmente a posição da primeira curva invariante spanning, podemos confirmar uma invariância de escala do histograma de frequências. O mesmo ocorre para a probabilidade de sobrevivência da partícula à dinâmica. Neste contexto, abordaremos os seguintes problemas: (1) Um guia de ondas senoidalmente corrugado; (2) Uma família de mapas Hamiltonianos bidimensionais que recupera diversos modelos; (3) Partículas confinadas em uma caixa com potenciais infinitos nas bordas e contendo em seu interior um poço de potencial dependente periodicamente do tempo; (4) Analisaremos um bilhar ovóide com dependência temporal introduzida através de giro, onde para certas condições observamos que este não apresenta um aparente crescimento ilimitado de energia (aceleração de Fermi), desta forma sendo um possível contra-exemplo da conjectura LRA. Esta tese é um resumo de 8 artigos que foram publicados em revistas internacionais.

Palavras-chave: caos, sistemas dinâmicos, bilhares, propriedades de escape, Aceleração de Fermi 


\section{Abstract}

We investigate some dynamical and transport properties for a set of non-interacting classical particles. The systems here described, for the most part, present mixed structure in the phase space in the sense that invariant spanning curves, chaotic seas and periodic islands are present. The dynamics of each model is described by using nonlinear mappings. We show all the details to construct the mappings and discuss some of their dynamical properties including fixed points stability among others. Lyapunov exponents will be obtained to characterize the chaotic dynamics observed in the phase space. Moreover some scaling hypotheses are used to prove that certain observables, including the average energy, are scaling invariant. We consider also that when a particle or an ensemble of them reach a certain portion of the phase space, they can escape. When studying the escape, we see that the histogram for the number of particles that reach certain height (or energy) $h$ in the phase space for the iteration $n$, for which we observe to be scaling invariant, grows quickly until reaching a maximum and then goes towards zero for large enough $n$. When changing the height $h$ proportionally to the position of the first invariant spanning curve, we can confirm the scaling invariance. The same happens for the survival probability for a particle in the chaotic dynamics. In this way, we will discuss the following problems: (1) A corrugated waveguide; (2) A family of two-dimensional Hamiltonian mappings which can reproduce different scaling exponents; (3) Particles confined to bounce in the interior of a time-dependent potential well; (4) We will analyse a rotating oval billiard, where for certain conditions we observed that this system does not present the unbounded energy growth (Fermi acceleration), in this way it is a possible counterexample of the LRA conjecture. This thesis is as summary of eight papers already published.

Keywords: chaos, dynamical systems, billiards, escape properties, Fermi acceleration 


\section{Sumário}

1 Introdução 1

1.1 Proposta e organização da tese . . . . . . . . . . . . . . . . 3

$\begin{array}{lll}2 & \text { Guia de ondas periodicamente corrugado } & \mathbf{7}\end{array}$

2.1 Resumo . . . . . . . . . . . . . . . . . . . 7

2.2 Motivação . . . . . . . . . . . . . . . . . . . . . . 8

2.3 O modelo e o mapeamento . . . . . . . . . . . . . . . 8

2.4 Resultados numéricos . . . . . . . . . . . . . . . . . . 10

2.5 Escape no guia de ondas . . . . . . . . . . . . . . . . . . 13

2.6 Resultados numéricos - Versão simplificada do mapeamento . . . . . . . 20

2.7 Conclusões . . . . . . . . . . . . . . . . . . . . . . . . . . . 23

3 Invariância de escala do coeficiente de difusão em uma família de mapeamentos Hamiltonianos bidimensionais $\quad 25$

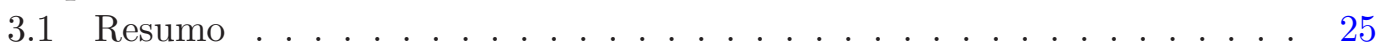

3.2 Motivação . . . . . . . . . . . . . . . . . . . 2 25

3.3 Coeficiente de difusão . . . . . . . . . . . . . . . . . . 27

3.4 Propriedades de transporte e resultados . . . . . . . . . . . . . . . 29

3.5 Conclusões . . . . . . . . . . . . . . . . . . . . . 33

4 Poço de potencial dependente periodicamente do tempo 35

4.1 Resumo . . . . . . . . . . . . . . . . . . . 35

4.2 Motivação . . . . . . . . . . . . . . . . . . . . . . 35

4.3 O modelo e o mapa . . . . . . . . . . . . . . . . . . . . 36

4.4 Espaço de fase e pontos fixos . . . . . . . . . . . . . . . . . . 39

4.5 Resultados - Propriedades de transporte através do mar caótico . . . . . 41

4.6 Conclusões parciais . . . . . . . . . . . . . . . . . . 45

4.7 Poço de potencial com diferentes perturbações temporais . . . . . . . . . . 45

4.7.1 Desvio da energia média ao longo do mar caótico . . . . . . . . 49

4.7.2 Localização da curva invariante spanning . . . . . . . . . . . . . 51 
4.7.3 Comportamento da energia como função do parâmetro $r$. . . . 51

4.7.4 Resultados para a função $f_{4}(\phi) \ldots \ldots \ldots$. . . . . . . . 54

4.8 Conclusões . . . . . . . . . . . . . . . . . . . 55

$\begin{array}{llr}5 & \text { Bilhar ovóide girante } & \mathbf{5 7}\end{array}$

5.1 Resumo . . . . . . . . . . . . . . . . . . 57

5.2 Motivação . . . . . . . . . . . . . . . . . . . . . . . 57

5.3 O modelo e o mapa . . . . . . . . . . . . . . . . . . . 58

5.4 Resultados numéricos . . . . . . . . . . . . . . . . . . 60

5.4 .1 Caso $(\mathrm{i}): \epsilon<\epsilon_{c} \ldots \ldots \ldots \ldots \ldots$. . . . . . . . . . 61

5.4 .2 Caso (ii): $\epsilon>\epsilon_{c} \ldots \ldots \ldots \ldots \ldots \ldots$

5.5 Conclusões . . . . . . . . . . . . . . . . . . . 66

$\begin{array}{lll}6 & \text { Conclusões finais } & 67\end{array}$

$\begin{array}{lll}7 & \text { Perspectivas futuras } & 69\end{array}$

$\begin{array}{ll}\text { Lista de Figuras } & 71\end{array}$

$\begin{array}{ll}\text { Lista de Tabelas } & 77\end{array}$

$\begin{array}{lr}\text { Bibliografia } & 79\end{array}$ 
$\Gamma_{\text {Capítulo } 1 \longrightarrow}$

\section{Introdução}

Um sistema é considerado caótico quando possui sensibilidade às condições iniciais. Isso implica que duas condições iniciais muito próximas divergem exponencialmente uma da outra ao longo do tempo. Uma ferramenta comumente utilizada para verificar a caoticidade de um sistema é o expoente de Lyapunov $(1,2,3)$.

Em sistemas Hamiltonianos bidimensionais os expoentes de Lyapunov aparecem aos pares e com sinais contrários. Enfatizamos que sempre consideraremos o expoente positivo em nossas simulações quando existir. Se o maior expoente de Lyapunov é nulo ou menor que zero, dizemos que a órbita é regular ou quasi-periódica, porém se um dos expoentes de Lyapunov é positivo, a órbita é dita ser caótica. Já em sistemas dissipativos (que apresentam alguma forma de dissipação, como por exemplo, arrasto viscoso), a soma dos expoentes de Lyapunov é menor que zero.

Muitas vezes é possível trabalharmos com mapeamentos, que são sistemas dinâmicos discretizados no tempo. Para cada iteração de um dado mapa o sistema passa de um estado inicial $X_{n}$ para $X_{n+1}$ através de uma transformação $T$, que pode ser escrita como $X_{n+1}=T\left(X_{n}\right)$, onde $n$ representa a $n$-ésima iterada do mapeamento. Podemos considerar como exemplo de mapa unidimensional o mapa Logístico (4), que é escrito na forma $X_{n+1}=R X_{n}\left(1-X_{n}\right)$, onde $R$ é o parâmetro de controle e $X$ é a variável dinâmica do sistema. Este mapa é um exemplo de mapeamento unidimensional dissipativo, com isso, para um dado conjunto de condições iniciais, o valor de $X$ pode convergir à um atrator ${ }^{1}$ que pode ser caótico ou do tipo ponto fixo (5). Um ponto fixo de período 1 pode ser considerado como o ponto onde o valor da variável é independente das iteradas, ou seja, $X_{n+1}=X_{n}$ para qualquer valor de $n$.

Consideraremos uma órbita o conjunto $X_{0}, X_{1}, X_{2}, X_{3}, \ldots, X_{m}$ obtido após $m$ iteradas de um mapeamento (6). Já um espaço de fases é o conjunto de diferentes órbitas, com diferentes condições iniciais.

Dizemos que o espaço de fases é misto quando contém ilhas periódicas, mares caóticos e curvas invariantes do tipo spanning que limitam ou separam diferentes regiões

\footnotetext{
${ }^{1}$ Atrator pode ser considerado um conjunto invariante para o qual órbitas próximas convergem depois de um tempo suficientemente longo.
} 


\section{INTRODUÇÃO}

caóticas. No interior das ilhas periódicas temos órbitas com movimento quasi-periódico ou regular. A evolução de uma condição inicial dentro das ilhas periódicas jamais fará com que a partícula saia desta região e atinja o mar caótico.

Uma classe de sistemas que pode ser descrita, consiste em considerar o problema do movimento livre de uma partícula clássica confinada em uma região fechada do espaço e que sofre colisões com a fronteira. Tais problemas são chamados de problemas do tipo bilhar (2). Estes sistemas podem ser discutidos e tratados, por exemplo, a partir de poços ou barreiras de potencial. Por volta do ano 1920, o conceito de bilhares foi introduzido dentro da teoria de sistemas dinâmicos por Birkhoff (7). A ideia de Birkhoff era ter uma classe simples de sistemas Hamiltonianos sem a necessidade de integrar equações diferenciais $(7,8)$. O sistema dinâmico discreto associado é conservativo e pode apresentar (dependendo da forma do bilhar) regimes dinâmicos como integrabilidade, não-integrabilidade e caos. Quando o potencial que descreve a região fechada é dependente do tempo ou de maneira equivalente, quando a posição da fronteira é dependente do tempo (bilhares pulsantes), temos exemplos simples de sistemas Hamiltonianos dependentes do tempo que também são colocados facilmente na forma de um sistema dinâmico discreto. Grande parte destes sistemas apresentam um espaço de fase do tipo misto. Especial atenção tem sido aplicada no comportamento caótico destes sistemas (9) assim como a possibilidade de caracterizá-los usando teoria de escala (10). Além do mais, alguns destes sistemas podem apresentar comportamentos descritos por leis de potência (11) com mesmos expoentes, os quais podem ser uma clara evidência de que estes sistemas podem ser enquadrados em uma mesma classe de universalidade.

Atualmente, muitos problemas envolvendo bilhares com orifícios em sua borda tem sido estudados, um exemplo é o bilhar em forma de limão (12), que possui espaço de fase misto e o tempo médio de escape depende da posição e geometria do buraco. Trabalhos recentes enfatizam a probabilidade de sobrevivência e escape de partículas em bilhares, como o caso do bilhar stadium $(13,14)$, bilhar circular aberto $(15)$, transporte assimétrico no modelo Bouncer (16) e os recentes avanços em bilhares abertos (17).

Um fenômeno bastante estudado em sistemas dinâmicos e bilhares é o processo onde uma partícula clássica adquire crescimento ilimitado de energia, chamado aceleração de Fermi (AF). Este processo foi reportado pela primeira vez por Enrico Fermi (18) como uma tentativa para explicar a aceleração de raios cósmicos. Ele propôs que tal comportamento aparece devido a interação entre partículas carregadas e um campo magnético dependente do tempo produzidos pelo meio interestelar. Mais tarde alguns outros modelos alternativos foram também propostos usando diferentes métodos com aplicações em diferentes áreas, incluindo física molecular(19), quantum dots(20), nanoestruturas(21) e outros. Entre diferentes sistemas, um dos mais destacados é o chamado modelo Fermi-Ulam. Este consiste de uma partícula clássica, ou um conjunto de partículas não-interagentes, confinadas entre duas paredes rígidas $(10,22,23,24)$. Uma delas se move no tempo, portanto correspondendo à variação do campo magnético e a outra é fixa. A funcionalidade da segunda parede é somente para produzir um mecanismo de reinjeção da partícula após a colisão com a parede móvel. A existência de AF no modelo Fermi-Ulam se deve exclusivamente a forma com que ocorre a lei de 
reflexão com a parede móvel( 3,25$)$, e contanto que esta lei seja suficientemente suave, curvas invariantes do tipo spanning pró́bem o crescimento ilimitado de energia das partículas.

Uma extensão natural do modelo Fermi-Ulam é o modelo Bouncer, que consiste de uma partícula livre, que está caindo sob a influência de um campo gravitacional constante e sofre a influência de uma parede móvel com massa infinita $(26,27)$. Este modelo é um bom exemplo de bilhar unidimensional que apresenta aceleração de Fermi.

\subsection{Proposta e organização da tese}

Afim de facilitar e melhor organizar esta tese, em cada seção destacaremos parte das motivações que levaram ao estudo de cada um dos sistemas aqui propostos. Os modelos e mapeamentos serão detalhados e os resultados publicados em revistas internacionais serão apresentados. Também mostraremos conclusões parciais sobre os resultados obtidos.

Podemos citar como um dos pontos em comum desta tese o fato de conseguirmos mostrar invariâncias de escala para diferentes observáveis em diferentes modelos. Alguns exemplos de invariância de escala que serão vistos aqui ocorrem na energia média ou desvio da energia média; no histograma do número de partículas que atingem certa altura $h$ no espaço de fases; na probabilidade de sobrevivência; no coeficiente de difusão. Invariâncias de escala são importantes pois mostram que após reescalas apropriadas, alguns observáveis médios no espaço de fases tem comportamentos universais.

Nesta tese estudaremos algumas propriedades dinâmicas e de escape para diferentes modelos. O espaço de fases dos modelos, em sua maioria, são mistos, contendo ilhas periódicas, mares caóticos e curvas invariantes do tipo spanning. Organizaremos esta tese da seguinte forma: no capítulo 2 mostraremos os resultados para um guia de ondas periodicamente corrugado. Neste modelo consideramos a dinâmica de um raio de luz que se move entre duas fronteiras, sendo uma delas plana e a outra com uma corrugação que depende de um único parâmetro de controle. Neste capítulo discutiremos os resultados obtidos e publicados em 3 artigos científicos que lidam com escape de partículas e propriedades dinâmicas de um guia de ondas $(28,29,30)$. Estudaremos tanto a versão completa como simplificada de tal modelo. A versão simplificada do modelo não nos mostra informações sobre reflexões múltiplas com a fronteira corrugada, porém facilita as nossas análises numéricas, principalmente para explicar de forma analítica a posição da primeira curva invariante spanning. Parte da nossa contribuição original neste assunto consiste em mostrar que a frequência de reflexões múltiplas é invariante de escala ao obtermos alguns expoentes de escala. Também mostraremos invariâncias de escala no desvio do ângulo médio. Outro ponto a se destacar será apresentar o guia de ondas inserido em meios com diferentes índices de refração e mostraremos que existe uma expressão analítica que descreve em quais situações teríamos o escape do raio de luz ao considerarmos diferentes meios materiais. Aqui mostraremos invariâncias de escala do histograma do número de feixes de luz que escapam em certa iterada $n$ em relação ao parâmetro de controle. Através de uma conexão com o mapa padrão e 


\section{INTRODUÇÃ̃O}

considerando o mapeamento simplificado, obteremos de forma analítica os expoentes de escala, onde este consequentemente nos mostra o comportamento da primeira curva invariante spanning. Para a versão simplificada do modelo também obteremos reescalas no histograma de frequência, onde a diferença agora será também mostrar reescalas para diferentes posições da região de escape.

No capítulo 3 analisaremos um mapeamento que reproduz uma família de mapas Hamiltonianos bidimensionais com diferentes expoentes. Particularmente discutiremos algumas propriedades de transporte e escape para um conjunto de partículas clássicas. Novamente nossa contribuição se deu, por exemplo, através de uma conexão com o mapa padrão, que é feita afim de obter analiticamente os expoentes de escala. Os coeficiente de difusão são obtidos considerando histogramas de frequência para o número de partículas que atingem uma certa altura no espaço de fases. Mostraremos que os coeficientes de difusão são invariantes de escala após reescalas apropriadas. Os resultados apresentados neste capítulo foram publicados em (31).

O capítulo 4 apresenta as discussões para um poço de potencial dependente periodicamente do tempo. Tal modelo mostra a dinâmica de um conjunto de partículas clássicas que sofre a influência de diferentes potenciais. Dependendo da região que a partícula se encontra e a fase do potencial móvel, é possível haver ganhos ou perdas de energia cinética. Este capítulo reúne resultados de 3 artigos que foram publicados recentemente $(32,33,34)$. Aqui nossa contribuição se destaca pelo fato de mostrarmos pela primeira vez na literatura que o histograma do número de partículas que escapam em uma dada iterada $n$ é invariante de escala (após reescalas apropriadas) ao considerarmos a posição do buraco proporcional à posição da primeira curva invariante spanning. Neste capítulo obteremos de forma analítica e numérica a posição dos pontos fixos elípticos e hiperbólicos no espaço de fases. Mostraremos também que a probabilidade de sobrevivência é invariante de escala frente a posição do buraco de escape. Na última parte deste capítulo mostraremos que os expoentes de escala não dependem das perturbações temporais propostas para o potencial móvel.

Já no capítulo 5 estudaremos um bilhar ovóide girante, que foi recentemente publicado (35). Este assunto é relativamente recente na literatura e podemos citar como uma de nossas contribuições o fato de termos observado que este modelo não apresenta aceleração de Fermi para certos valores dos parâmetros de controle, o que é uma aparente contradição da conjectura LRA. Veremos que as órbitas se movem através de curvas de Larmor, o que nos remete a uma analogia entre o bilhar girante e bilhares magnéticos. No capítulo 6 apresentaremos as conclusões finais sobre a tese. Finalmente, no capítulo 7 apresentaremos as perspectivas futuras e por fim as referências bibliográficas.

Foi escolhida esta ordem de apresentação dos modelos por se tratar de um gradativo aumento na complexidade dos sistemas. Por exemplo, no capítulo 2 tratamos do modelo do guia de ondas corrugado, tal sistema tem fronteiras estáticas e apenas 1 parâmetro de controle que rege a dinâmica do sistema. No capítulo 3 apresentamos uma família de mapeamentos Hamiltonianos bidimensionais, e tal sistema apresenta 2 parâmetros de controle que podem ser ajustados. Já no capítulo 4, que trata do poço de potencial, o fundo do poço depende explicitamente do tempo, onde tal sistema possui um grau 
e meio de liberdade. Neste modelo, o número de parâmetros de controle estudados é igual à 3 . Finalmente, no capítulo 5 decidimos introduzir a dependência temporal na fronteira de um bilhar. A dependência temporal é inserida através do giro, o que faz com que o bilhar que antes era um mapa bidimensional em sua forma estática se torne um mapeamento quadridimensional. Todos os resultados que serão apresentados nesta tese são novos, originais e deverão contribuir para o avanço do conhecimento na área de sistemas dinâmicos. 
1. INTRODUÇÃO 


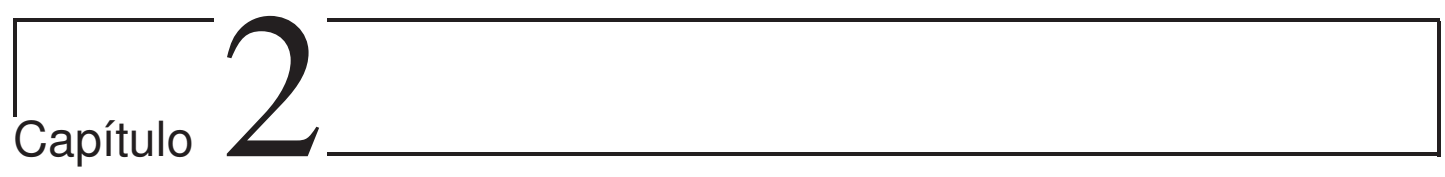

\section{Guia de ondas periodicamente corrugado}

\subsection{Resumo}

Neste capítulo estudaremos algumas propriedades dinâmicas e de escape de um raio de luz inserido em um guia de ondas senoidalmente corrugado. O raio é confinado entre dois espelhos, onde um deles é plano e o outro é senoidalmente corrugado. O sistema é controlado por um parâmetro de corrugação $\delta$ que controla a transição de integrável $(\delta=0)$ para não-integrável $(\delta \neq 0)$. A evolução do sistema é descrita pelo uso de um mapa bidimensional não linear. Para $\delta \neq 0$ o espaço de fases é misto, contendo ilhas periódicas, regiões de comportamento caótico e curvas invariantes do tipo spanning. Caracterizamos a probabilidade de se observar reflexões sucessivas de um raio de luz no espelho corrugado e mostramos que esta é invariante de escala com relação à amplitude de corrugação. Propriedades médias de observáveis ao longo do mar caótico também são descritas pelo uso de argumentos de escala. Na seção do escape, o guia de ondas será inserido dentro de uma região com índice de refração diferente e utilizamos as equações de Fresnel, que mostram as condições de refração e consequentemente de escape do raio do guia de ondas. Os resultados são discutidos usando parâmetros de controle que são fisicamente motivados em diferentes materiais ópticos para estimar em que situações ocorre o escape. Finalmente, na última seção mostraremos os resultados para a versão simplificada desde modelo, onde algumas aproximações são necessárias para descrever o mapa.

Este capítulo é um resumo de três artigos, um deles foi publicado em 2012 na Journal of Physics A, Mathematical and Theoretical (28). Outro artigo foi recentemente publicado no Physics Letters A (29) em 2012. Por fim, o último foi publicado na revista Communications in Nonlinear Science $\&$ Numerical Simulation (30) e deve sair em 2014. 


\section{GUIA DE ONDAS PERIODICAMENTE CORRUGADO}

\subsection{Motivação}

Nos últimos anos o interesse em guias de onda ou canais corrugados tem aumentado. Ambos os tratamentos clássicos e quânticos estão sendo investigados por vários autores, além de experimentações. Aplicações envolvendo este assunto levam em conta diferentes sistemas incluindo uma técnica para detectar uma grande fração de fótons emitido por diferentes centros de cor dentro de um diamante plano (36) e dispersão determinística de uma partícula quântica em um guia de ondas balístico bidimensional. Propagação de pulso e aceleração de elétrons em um plasma corrugado são também exemplos de possíveis aplicações. Historicamente, aceleração direta de partículas carregadas por campos eletromagnéticos têm sido limitada por correspondência de fases, difração e limite de dano nos materiais, mas agora cientistas tem uma alternativa para produzir elétrons rápidos usando um plasma de micro-óptica $(37,38)$. Além disso, aplicações também podem envolver o transporte através de uma heteroestrutura finita do GaAs $/ \mathrm{Al}_{\mathrm{x}} \mathrm{Ga}_{1-\mathrm{x}} \mathrm{As}(39)$, acústica subaquática $(40,41,42)$ e muitas outras aplicações (37). Diante da importância de tais modelos, nos propomos a estudar a dinâmica de um raio de luz inserido em um guia de ondas corrugado com vistas à compreender o comportamento caótico das órbitas no espaço de fases. Como veremos, o modelo aqui abordado apresenta um espaço de fases misto, que contêm diversas informações relevantes para a caracterização de tal sistema. As seções a seguir descrevem os resultados publicados em 3 revistas internacionais $(28,29,30)$. Ao longo de cada seção mostraremos quais foram alguns dos principais resultados obtidos nos artigos.

\subsection{O modelo e o mapeamento}

Nesta seção descreveremos todos os passos para construir as equações do mapeamento que descrevem a dinâmica do sistema. O modelo consiste basicamente de um raio de luz que é confinado e sofre reflexões entre dois espelhos. Um deles é plano e localizado na posição $y=y_{0}$ enquanto o outro é dado pela equação $y=d \cos (k x)$, onde $d$ é a amplitude de corrugação, $k$ é o número de onda e $x$ é a posição ao longo do espelho. Os ângulos envolvidos na reflexão são obtidos assumindo reflexões especulares, ou seja, o ângulo em relação à normal do raio incidente é igual ao do raio refletido. Temos três parâmetros de controle no total, mas o sistema não depende de todos eles. Por isso, torna-se necessário definirmos variáveis adimensionais. Definiremos $X=k x(\bmod 2 \pi)$, $\delta=d / y_{0}$ e $Y=y / y_{0}$, então o espelho corrugado é dado por $Y=\delta \cos (X)$, onde $X$ é a fase ao longo do canal corrugado, e com isso, o espelho plano estará na posição $Y=1$. Um esquema ilustrativo do guia de ondas é mostrado na figura 2.1.

O mapa, nas novas variáveis, é dado pela variável $A_{n}$, que é o ângulo do raio refletido e que é medido a partir da vertical (sentido horário será considerado ângulo positivo). A segunda variável do sistema é $X_{n}$, que é a fase de reflexão no espelho corrugado, onde $n$ corresponde a enésima reflexão do raio. A figura 2.1 mostra dois casos típicos de reflexões com ângulos positivos: (1) a reflexão ocorre depois que o raio é refletido no espelho corrugado e; (2) a reflexão ocorre depois que o raio é refletido pelo espelho 


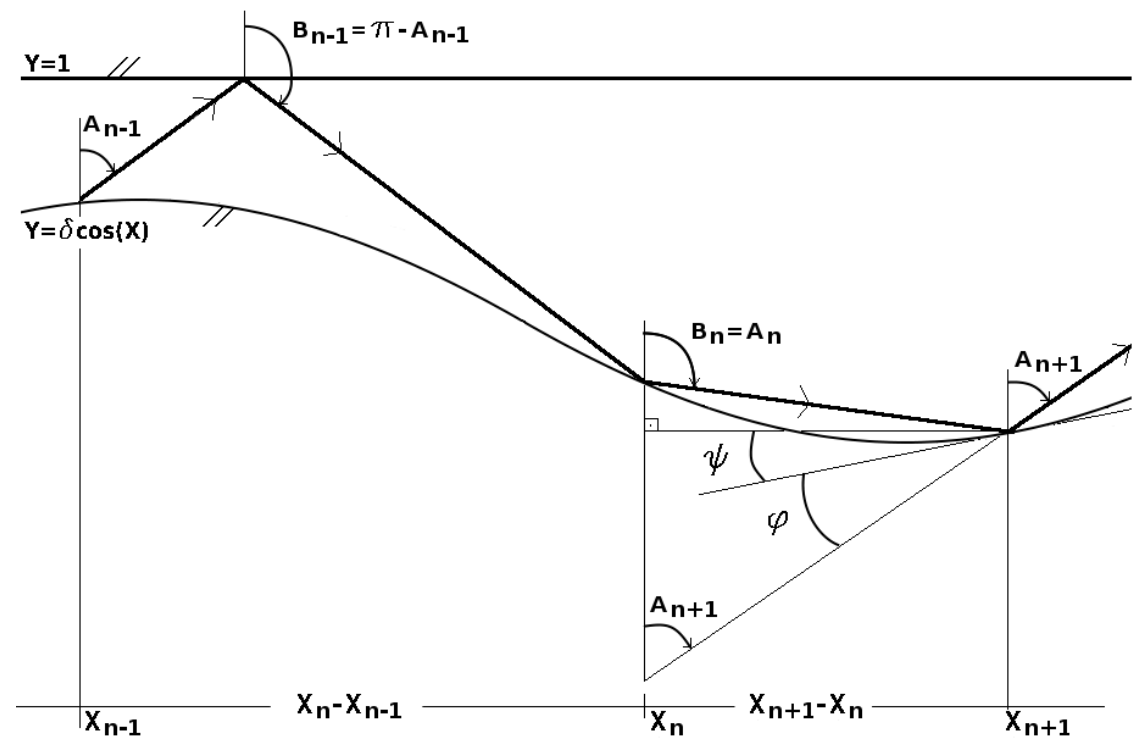

Figura 2.1: Esquema ilustrativo do guia de ondas e dos ângulos envolvidos em sua descrição.

plano. Para o caso (1) assumimos que o raio começa sua trajetória a partir do espelho corrugado na fase $X_{n}$, viaja a distância $\Delta Y=\delta \cos \left(X_{n+1}\right)-\delta \cos \left(X_{n}\right)$ e é refletido novamente. Portanto, $X_{n+1}$ é obtido numericamente através da solução da equação transcendental

$$
\frac{X_{n+1}-X_{n}}{\delta \cos \left(X_{n+1}\right)-\delta \cos \left(X_{n}\right)}=\tan \left(A_{n}\right) .
$$

O caso (2) é utilizado quando o raio deixa o espelho corrugado $\left(X_{n-1}\right)$ e viaja em direção ao espelho plano. Depois de sofrer uma reflexão especular o raio viaja de volta em direção ao espelho corrugado $\left(X_{n}\right)$. A fase de reflexão é obtida resolvendo numericamente a seguinte equação:

$$
\frac{X_{n+1}-X_{n}}{1-\delta \cos \left(X_{n+1}\right)+1-\delta \cos \left(X_{n}\right)}=\tan \left(A_{n}\right)
$$

É importante ressaltar que a zona de reflexão é definida como a região onde $Y \in[-\delta, \delta]$.

O ângulo do raio refletido $A_{n+1}$ pode ser obtido do vetor tangente na fase de reflexão. Portanto, para $A_{n}>0$ e de propriedades geométricas, encontramos que $B_{n}=\pi / 2+$ $\varphi-\psi_{n}$ e $A_{n+1}=\pi / 2-\varphi-\psi_{n+1}$, onde $\psi_{n+1}$ é o arco medido no sentido anti-horário a partir do eixo $X$ positivo até a tangente. O coeficiente angular para a tangente é de fato igual a derivada da função que descreve a superfície corrugada do espelho avaliada em $X_{n}$. Eliminando $\varphi$ obtemos que $A_{n+1}=-B_{n}-2 \psi_{n+1}$ onde: (1) $B_{n}=A_{n}$ se o raio vem do espelho corrugado e; (2) $B_{n}=\pi-A_{n}$ se o raio vem do espelho plano. Similarmente se $A_{n}<0$ nós temos que $B_{n}=A_{n}$ se o raio vêm do espelho corrugado e $B_{n}=-\pi-A_{n}$ se o raio vêm do espelho plano, com isso teremos que $A_{n+1}=-\pi-B_{n}-2 \psi_{n+1}$. 


\section{GUIA DE ONDAS PERIODICAMENTE CORRUGADO}

No artigo publicado na revista J. Phys. A: Math. and Theor. (Ref. (28)) mostramos com detalhes uma forma de se resolver as equações transcendentais que otimizam os programas usados nas análises numéricas, desta forma tornando os programas muito mais rápidos e também não perdendo nenhuma das soluções.

\subsection{Resultados numéricos}

O espaço de fases para o modelo é mostrado na figura 2.2(a), porém é mais conveniente expressar o ângulo do raio refletido como $\theta=\pi / 2-A$. Para o item (a) temos que
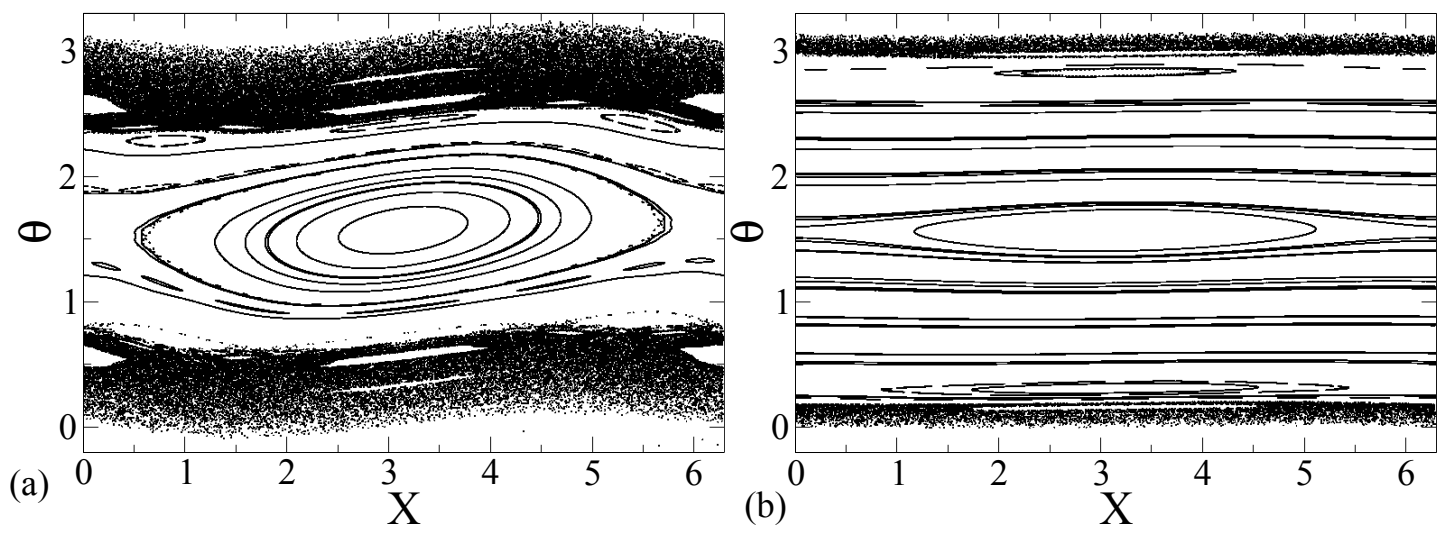

Figura 2.2: Espaço de fases para: (a) $\delta=0,1$; (b) $\delta=0,01$. Podemos ver que o tamanho ocupado pelo espaço de fases depende do parâmetro $\delta$.

$\delta=0,1$ e para o item (b) temos $\delta=0,01$. Podemos ver que a estrutura do espaço de fases é mista, apresentando ilhas periódicas, curvas invariantes spanning e mares caóticos.

Durante a dinâmica do raio de luz, reflexões sucessivas podem ocorrer no espelho corrugado. Estes eventos são raros ao longo da dinâmica e a probabilidade de se observar uma reflexão sucessiva é maior do que duas, que é maior do que se observar três, e assim por diante. Uma distribuição de probabilidade $(P)$ para $10^{10}$ iteradas do mapa considerando uma única condição inicial em função do número de reflexões $n_{s r}$ é mostrada na figura 2.3(a) para diferentes valores de $\delta$. O comportamento de $P$ é dado por uma lei de potência do tipo $P \propto n_{s r}^{\gamma}$, onde uma análise numérica nos forneceu $\gamma=-3,76(3)$, onde o índice $s r$ representa reflexões sucessivas. Como todas as curvas tem mesma inclinação, isto é um indício de que o comportamento de $P$ pode ser invariante de escala para $\delta$. Para observar isso, escolhemos uma probabilidade $P$ particular, por exemplo, $P=10^{-6}$ e obtivemos o correspondente valor de $n_{s r}$ para diferentes valores de $\delta$, onde isso é mostrado na figura 2.4. Um ajuste em lei de potência nos forneceu o expoente $0,252(4) \approx-1 / \gamma$.

Com o expoente obtido na figura 2.4, uma reescala pode ser feita na figura 2.3(a) $\left(n_{s r} \rightarrow n_{s r} / \delta^{0,252}\right)$, conforme mostramos na figura 2.3(b). Como as curvas colapsam uma sobre as outras, isto confirma que as curvas são invariantes de escala em relação 

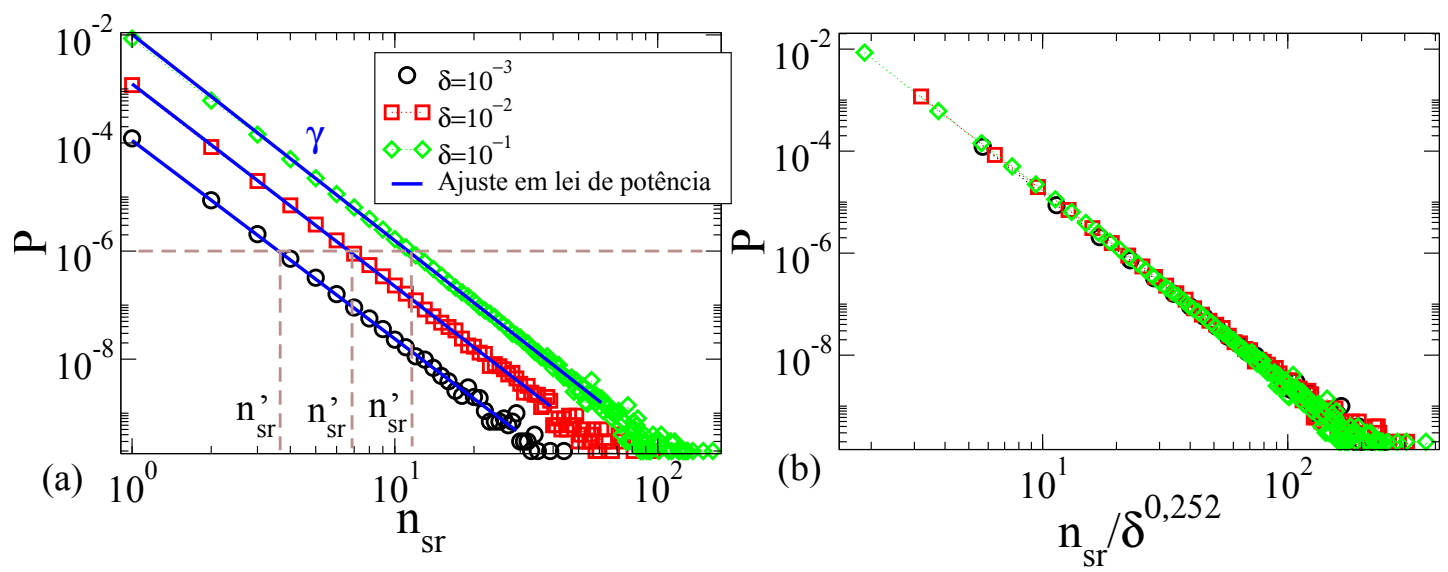

Figura 2.3: (a) Gráfico de $P \times n_{s r}$ para: $\delta=10^{-3}, \delta=10^{-2}$ e $\delta=10^{-1}$. (b) Depois de uma reescala $\left(n_{s r} \rightarrow n_{s r} / \delta^{0,252}\right)$, todas as curvas de (a) colapsam uma sobre as outras em uma única curva universal.

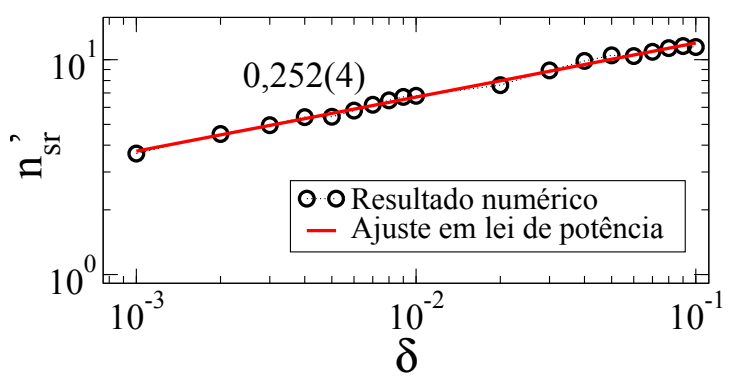

Figura 2.4: Gráfico de $n_{s r}^{\prime} \times \delta$. Um ajuste em lei de potência nos fornece como inclinação $0,252(4)$.

ao parâmetro de controle $\delta$. Isso implica que para qualquer valor de $\delta$ escolhido o comportamento de $P$ será sempre o mesmo.

Agora estudaremos o comportamento do desvio do ângulo de reflexão médio. Temos que o ângulo de reflexão médio é dado por $\bar{\theta}(n, \delta)=\frac{1}{n} \sum_{i=1}^{n} \theta_{i}$ e o desvio do ângulo

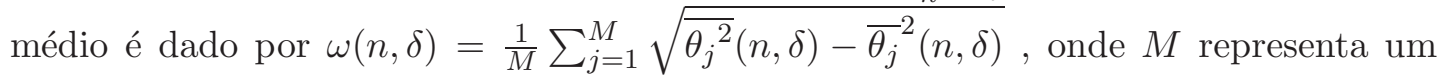
conjunto de diferentes condições iniciais que foram escolhidas considerando $\theta_{0}=\delta \mathrm{e}$ $X_{0} \in(0,2 \pi]$.

Para termos uma boa estatística de $\omega$ mas ao mesmo tempo um razoável tempo computacional, investigamos o comportamento da razão desvio padrão, $\Delta \omega$, sobre a média do conjunto $\omega$, i.e. $R=\Delta \omega / \bar{\omega}$. A figura 2.5 mostra um gráfico de $R$ em função do tamanho do conjunto. Concluímos que $M=500$ condições iniciais geradas para um conjunto é suficiente para estabilizar a métrica $R$ para qualquer $n \in[10,10000]$. Por segurança iremos considerar $M=1000$.

Um gráfico típico de $\omega \times n$ é mostrado na figura 2.6(a). Vemos que $\omega$ inicia um crescimento em lei de potência do tipo $\omega \propto n^{\beta}$ seguido de uma saturação $\left(\omega_{\text {sat }}\right)$ após 


\section{GUIA DE ONDAS PERIODICAMENTE CORRUGADO}

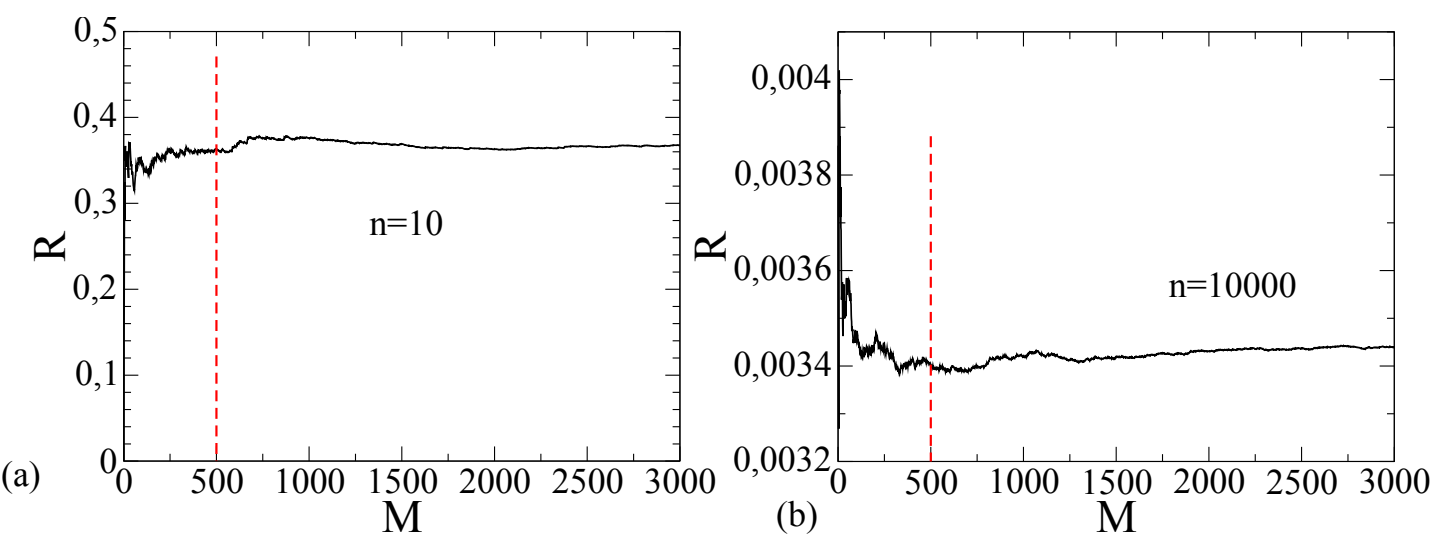

Figura 2.5: Gráfico de $\Delta \omega / \omega \times M$ para: (a) $n=10$ colisões; (b) $n=10000$ colisões. Mesmos comportamentos foram obtidos para diversos valores de $n \in[10,10000]$.
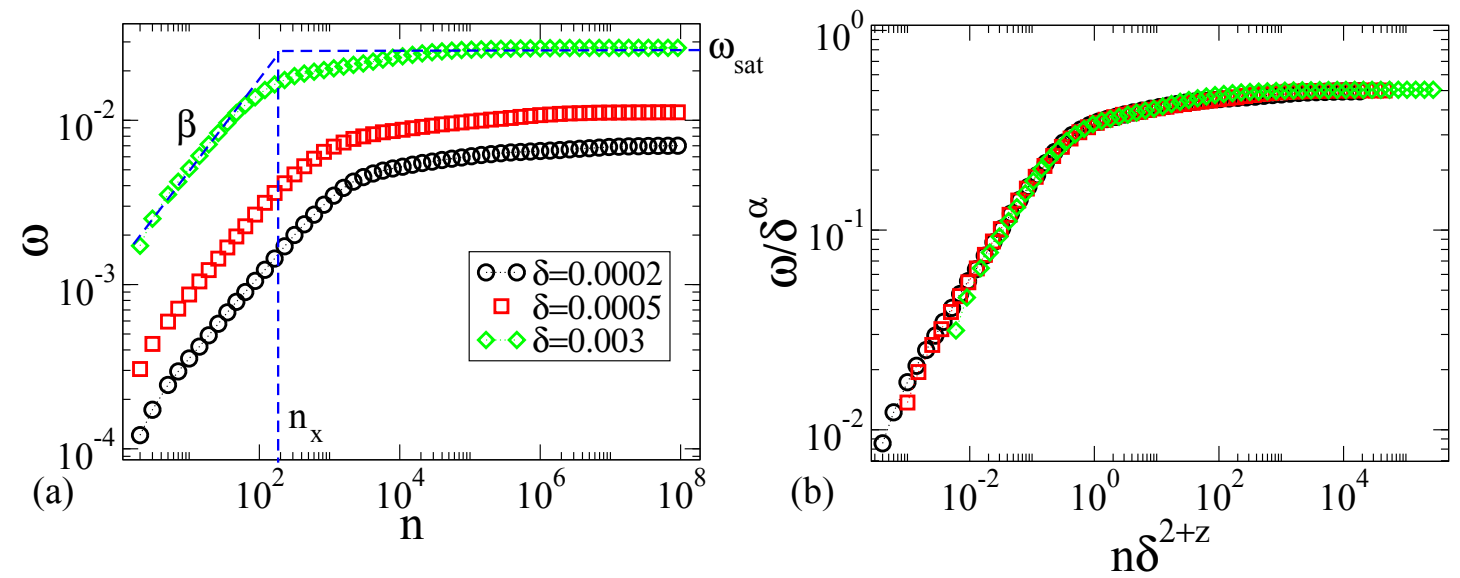

Figura 2.6: Gráfico de $\omega \times n$ para os parâmetros $\delta=2 \times 10^{-4}, \delta=5 \times 10^{-4}$ e $\delta=3 \times 10^{-3}$; (b) Depois de uma reescala $\left(n \rightarrow n \delta^{2+z}\right.$ e $\left.\omega \rightarrow \omega / \delta^{\alpha}\right)$ todas as curvas de (a) colapsam em uma única curva universal. Usamos um conjunto de $M=1000$ condições iniciais.

um número de crossover $n_{x}$. O comportamento de $\omega$ no regime de saturação é dado por $\omega \propto \delta^{\alpha}$ ao passo que $n_{x} \propto \delta^{z}$.

Expoentes podem ser obtidos se o comportamento de $\omega_{\text {sat }}$ e $n_{x}$ são obtidos em função do parâmetro de controle. Após analisar várias curvas de $\omega$, encontramos que $\beta \cong 0,5$. Os expoentes em função de $\delta$ são mostrados nas figuras $2.7(\mathrm{a}, \mathrm{b})$. Os expoentes obtidos foram $\alpha=0,508(4) \cong 0,5$ e $z=-0,956(9) \cong-1$. De posse dos valores numéricos destes expoentes, uma reescala pode ser feita nos eixos da figura 2.6(a), com isso produzindo um único gráfico universal para diferentes valores de $\delta$, como é feito na figura 2.6(b), com isso provando uma invariância de escala. 

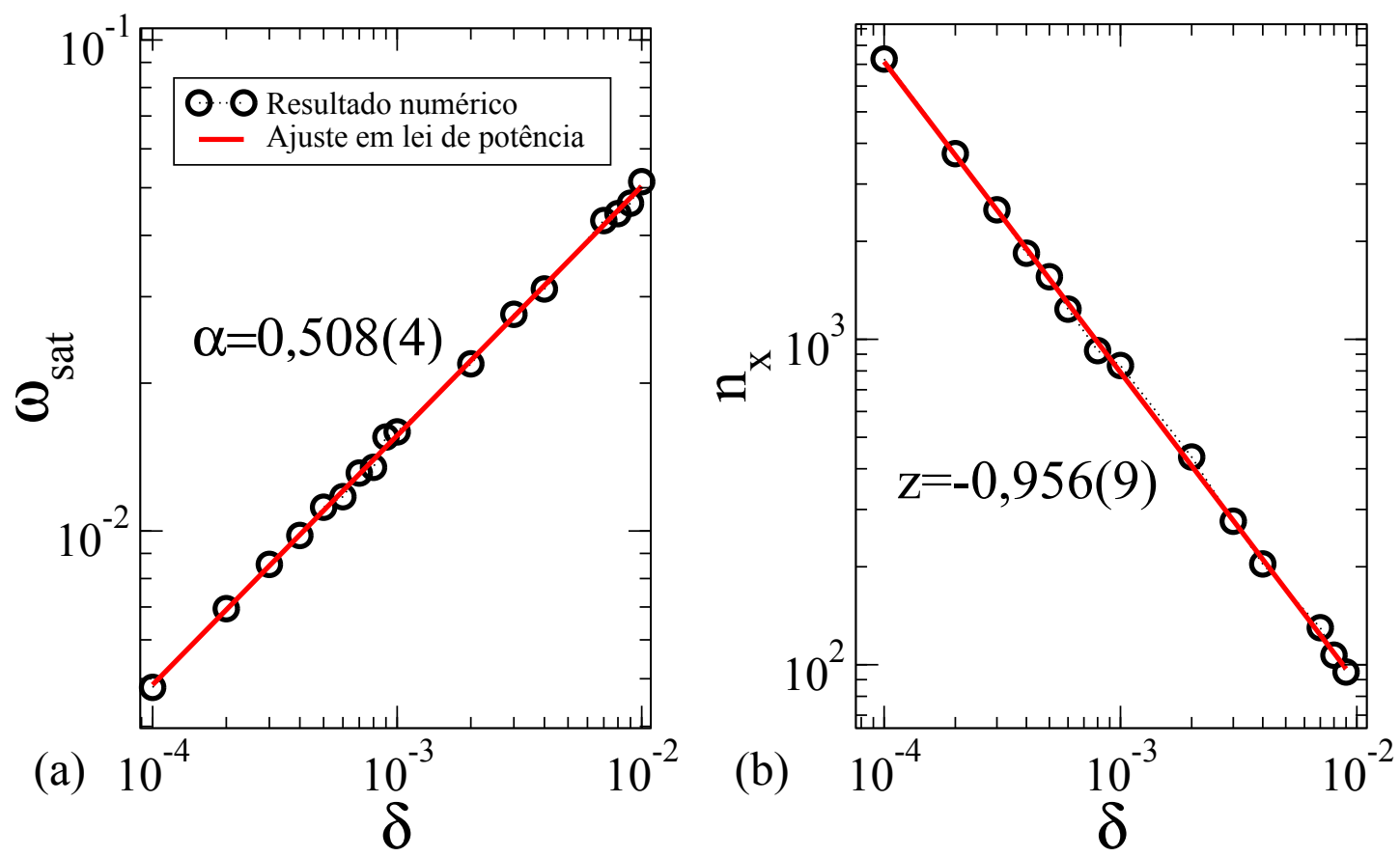

Figura 2.7: Para $M=1000$ : (a) Gráfico de $\omega_{\text {sat }} \times \delta$, onde o expoente encontrado foi $\alpha=0,508(4)$; (b) Resultados para $n_{x} \times \delta$, onde $z=-0,956(9)$.

\subsection{Escape no guia de ondas}

Agora destacaremos alguns dos resultados publicados na Ref. (30). Como veremos a seguir, este artigo apresenta alguns resultados sobre o escape de partículas (ou do feixe de luz) no guia de ondas, onde damos uma motivação física para as situações em que o raio de luz escapa.

As equações de Fresnel foram primeiramente obtidas no início do século 19 (30, 43) e descrevem o comportamento da luz se movendo em um meio com diferentes índices de refração. A reflexão de luz que as equações predizem é conhecida como reflexão de Fresnel. Quando a luz se move de um meio com índice $n_{1}$ rodeada por uma superfície com índice de refração $n_{2}>n_{1}$, reflexão e refração da luz podem ser observadas. As equações de Fresnel descrevem a fração da luz que é refletida (parte do raio escapa e é refratado) quando o ângulo de incidência $\theta_{i}$ (entre o raio incidente e o vetor normal à superfície no ponto de incidência) é menor que um ângulo crítico dado por

$$
A_{C}=\arcsin \left(n_{21}\right)
$$

onde $n_{21}=n_{2} / n_{1}$. Uma reflexão total ocorre se o raio permanece totalmente no guia de ondas para ângulos de incidência maiores do que o ângulo crítico. A fração da potência incidente é dada pela reflectância $R$ e esta é igual à unidade para o caso $\theta_{i}>A_{C}$. Entretanto, para $\theta_{i} \leq A_{C}$ os cálculos de $R$ dependem da polarização do raio incidente. Dois casos devem ser analisados: (i) a luz incidente é $p$-polarizada, 


\section{GUIA DE ONDAS PERIODICAMENTE CORRUGADO}
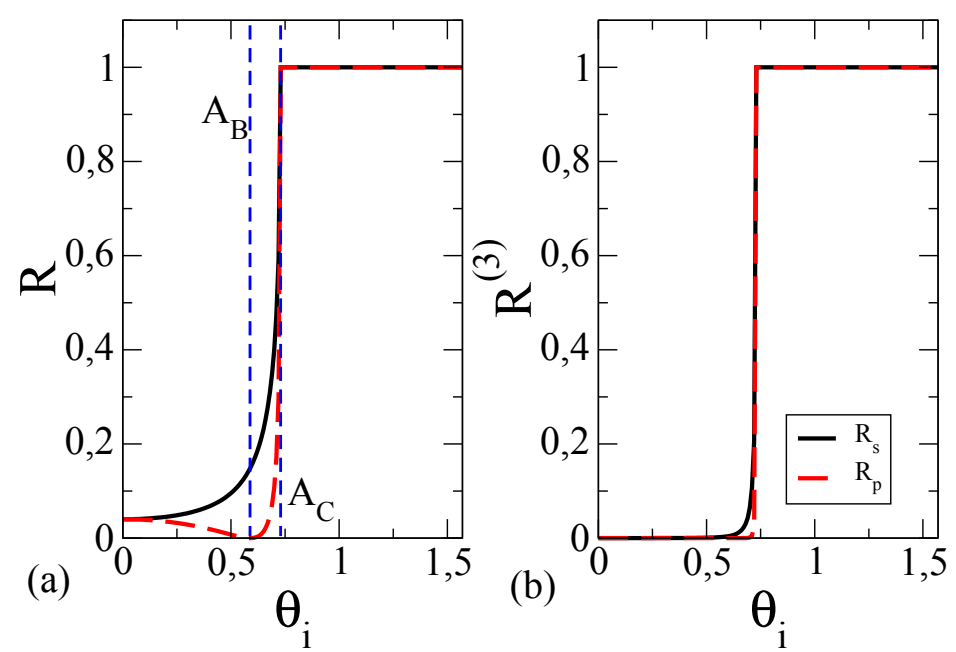

Figura 2.8: (a) Curvas de reflectância para raios s-polarizado (curva preta contínua) e $p$-polarizado (linha tracejada vermelha) em função do ângulo de incidência $\theta_{i}$. Foi considerado $n_{21}=n_{2} / n_{1}=1 / 1,5$; (b) Terceira potência da reflectância em função do ângulo de incidência $\theta_{i}$ para raios $s$-polarizado (curva continua preta) e $p$-polarizado (linha tracejada vermelha).

onde o campo elétrico está no plano incidente que contém o raio incidente e o vetor normal à superfície no ponto de incidência; (ii) a luz incidente é $s$-polarizada, onde o campo elétrico está em uma direção perpendicular à p-polarizada. Para um raio de luz $s$-polarizado, o coeficiente de reflexão é dado por

$$
R_{s}=\left(\frac{\cos \left(\theta_{i}\right)-n_{21} \sqrt{1-\left(\frac{\sin \left(\theta_{i}\right)}{n_{21}}\right)^{2}}}{\cos \left(\theta_{i}\right)+n_{21} \sqrt{1-\left(\frac{\sin \left(\theta_{i}\right)}{n_{21}}\right)^{2}}}\right)^{2},
$$

e para a luz p-polarizada, o coeficiente de reflexão é igual à

$$
R_{p}=\left(\frac{-n_{21} \cos \left(\theta_{i}\right)+\sqrt{1-\left(\frac{\sin \left(\theta_{i}\right)}{n_{21}}\right)^{2}}}{n_{21} \cos \left(\theta_{i}\right)+\sqrt{1-\left(\frac{\sin \left(\theta_{i}\right)}{n_{21}}\right)^{2}}}\right)^{2} .
$$

Estas equações podem ser usadas para outras polarizações do raio de luz incidente pela decomposição em $s$ e $p$. Por outro lado, quando o raio não é polarizado(isto é, a decomposição do raio tem média nula) o raio deve ser considerado contendo uma mistura equivalente destas polarizações. Neste caso, podemos ver pela figura 2.8(a) que depois da incidência, com ângulo menor que o crítico, o raio se torna predominantemente $s$-polarizado (polarização parcial). O raio pode ser totalmente $s$-polarizado se o ângulo de incidência é igual ao ângulo de Brewster $\left(A_{B}=\arctan \left(n_{21}\right)\right.$, isto é, $\left.\sin \left(A_{B}\right) / \cos \left(A_{B}\right)=n_{21}\right)(44,45,46)$, onde $R_{p}$ é nulo. 


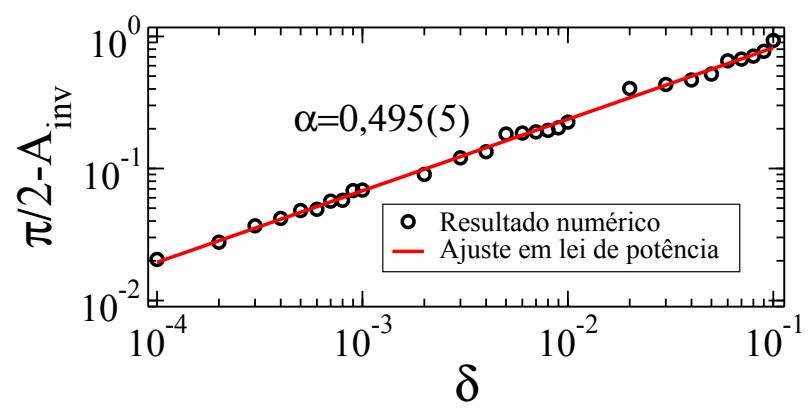

Figura 2.9: Comportamento da primeira curva invariante spanning $\left(A_{\text {inv }}\right)$ em função do parâmetro $\delta$. Um ajuste em lei de potência fornece $\left(\pi / 2-A_{\text {inv }}\right) \propto \delta^{\alpha} \operatorname{com} \alpha=0,495(5)$.

Na figura 2.9 temos $\left(\pi / 2-A_{\text {inv }}\right)$ em função do parâmetro $\delta$, onde $A_{\text {inv }}$ significa o ângulo médio na curva invariante spanning. Esta por sua vez é caracterizada por uma lei de potência com expoente $\alpha=0,495(5) \cong 0,5$, com isso temos uma expressão da forma

$$
\frac{\pi}{2}-A_{\text {inv }}=1,958 \delta^{\alpha} .
$$

Agora consideraremos que o guia de ondas está inserido em um meio com diferentes índices de refração, onde o índice de refração do guia de ondas é $n_{1}$ enquanto o de fora é $n_{2}<n_{1}$. Considerando inicialmente um raio não polarizado composto por dois raios $s$-polarizado e $p$-polarizado $I_{0}=I_{p 0}+I_{s 0}$ com mesmas intensidades $I_{p 0}=I_{s 0}$, a intensidade do raio dentro do guia de ondas após $n$ reflexões é dada por

$$
I_{n}=\left(\prod_{j=1}^{n} R_{p j}\right) \cdot I_{p 0}+\left(\prod_{j=1}^{n} R_{s j}\right) \cdot I_{s 0} .
$$

Considerando que $R_{s}$ não pode ser zero (ver a figura 2.8(a), onde podemos ver que $R_{s}$ não tem valor nulo para todo $\theta_{i} \in[0, \pi / 2)$ ), concluímos que o raio nunca escapa totalmente do guia de ondas. Entretanto, por propósitos práticos nós definiremos um limite, com isso, calculando as estatísticas de sobrevivência. A figura 2.8(b) mostra um gráfico da terceira potência da reflectância de Fresnel. Podemos ver que as curvas são aproximadamente funções degrau em $A_{C}$ (ângulo crítico). Se considerarmos uma função degrau ao invés da Eq. (2.7), não haverá mudanças significativas nos resultados. De fato em nossas simulações não foi observado mais do que três reflexões de Fresnel para que a intensidade caísse mais que $10^{-10}$ da intensidade inicial $I_{0}$. Como conclusão se $\theta_{i}>A_{C}$ o raio é completamente refletido e permanece confinado no guia de ondas. Por outro lado, se $\theta_{i} \leq A_{C}$ o raio é totalmente refratado e escapa do guia de ondas.

Definimos uma fração do espaço de fases, medido em relação à curva invariante spanning e o ângulo $A_{C}$. Esta fração é definida como $c_{p}$ e é escrita como

$$
c_{p}=\frac{A_{\mathrm{C}}-A_{\text {inv }}}{A_{0}-A_{\text {inv }}}
$$

onde $A_{0}$ corresponde ao ângulo inicial e $A_{C}$ o ângulo crítico. 


\section{GUIA DE ONDAS PERIODICAMENTE CORRUGADO}
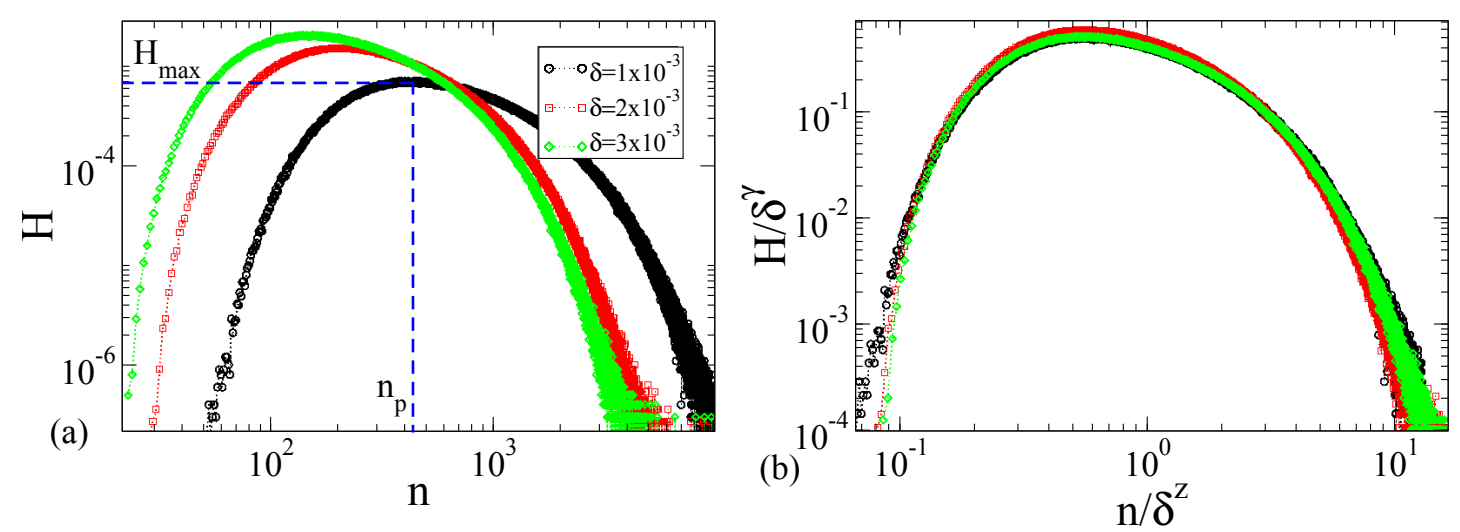

Figura 2.10: (a) Gráfico do histograma relativo de órbitas que escaparam para $c_{p}=0,3$ e três diferentes valores do parâmetro $\delta$. (b) Depois de uma reescala no eixo horizontal $\left(n \rightarrow n / \delta^{-0,963}\right)$ e vertical $\left(H \rightarrow H / \delta^{0,953}\right)$ todas as curvas mostradas em (a) colapsam em uma única curva universal.

Nossa primeira consideração assume que o escape ocorre apenas na superfície corrugada e a superfície lisa é um espelho perfeito. A figura 2.10(a) mostra o histograma de escape relativo $H$ para o número de raios que escaparam após $n$ reflexões na superfície corrugada do guia de ondas. Consideramos $c_{p}=0,3$ e usamos diferentes valores para $\delta$, como mostrado na figura. Outros valores de $c_{p}<1$ também foram considerados. Vemos que as curvas de $H$ inicialmente crescem, atingem um máximo $\left(H_{\max }\right)$ em $n=n_{p} \mathrm{e}$ decrescem atingindo o valor nulo para $n$ suficientemente longo.

O comportamento de $n_{p}$ em função de $\delta$ é mostrado na figura 2.11(a) onde consideramos diferentes valores de $c_{p}$ e temos o escape em ambas as fronteiras. Depois de aplicar um ajuste em lei de potência nas diversas curvas mostradas na figura 2.11(a) nós obtivemos que $\bar{z}=-0,963(4)$. Outro expoente pode ser obtido depois de analisar $H_{\max }$ em função de $\delta$, como mostrado na figura 2.11(b), onde temos $\bar{\gamma}=0,953(1)$. Estes expoentes podem ser usados para colapsar as diferentes curvas mostradas na figura 2.10(a) em uma única curva universal, como mostrado na figura 2.10(b), com isso, comprovando uma invariância de escala para o histograma. O último expoente $(\beta)$ pode ser encontrado indiretamente observando $n_{p}$ em função de $c_{p}$ (ver figura 2.11(c)), onde $\bar{u}=-2,05(3)=-1 / \beta$, desta forma temos que $\beta \cong 0,5$.

Vamos agora considerar um caso limite onde o ângulo de escape é definido na posição da curva invariante. Da Eq. (2.3) e Eq. (2.6) nós encontramos uma expressão para $n_{21}$ no caso limite como

$$
n_{21}=\frac{n_{2}}{n_{1}}=\sin \left(\frac{\pi}{2}-1,958 \delta^{\alpha}\right) .
$$

Para termos uma ideia dos ângulos limites de escape dados pela Eq. (2.9) e considerando diferentes índices, mostramos na figura 2.12 através da curva tracejada alguns vidros ópticos com índices $n_{1}$ (consideramos a água como referência) e rodeados por ar com índice de refração $n_{2} \cong 1$. A região acima da curva tracejada representa a situação onde o escape não é observado (refração). Os dados foram obtidos da Ref. (47). 

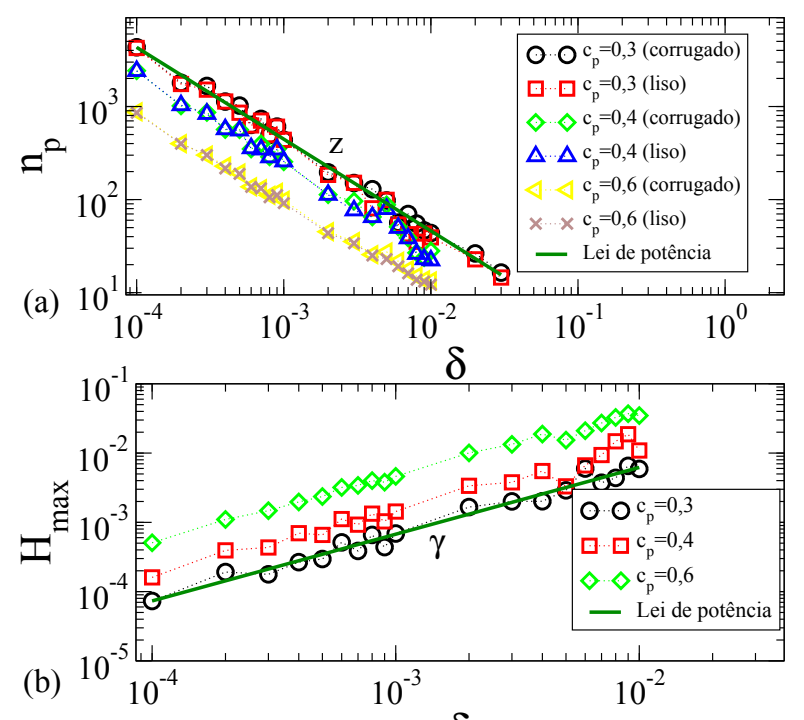

(b)

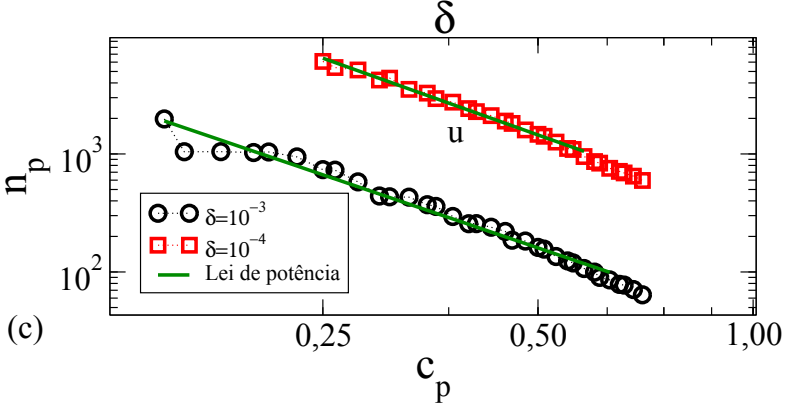

Figura 2.11: (a) Gráfico de $n_{p}$ em função de $\delta$ para diferentes valores de $c_{p}$ e considerando o escape em ambas partes lisa e corrugada do bilhar. Um ajuste em lei de potência fornece $z=-0,963(4) \cong-1 ;(\mathrm{b})$ Gráfico de $H_{\max }$ em função de $\delta$ para três diferentes valores de $c_{p}$. O expoente obtido foi $\gamma=0,953(1) \cong 1$; (c) Gráfico de $n_{p}$ em função de $c_{p}$ para $\delta=10^{-2}$ e $\delta=10^{-3}$, onde o expoente médio obtido foi $u=-2,05(3)$.

Discutimos agora a preferência de escape através da superfície plana ou corrugada. A figura 2.13(a,b) mostra a evolução de $10^{4}$ diferentes condições iniciais escolhidas como $A_{0}=\pi / 2-\delta$ e os valores de $X_{0}$ foram escolhidos aleatoriamente no intervalo $X_{0} \in[0,2 \pi)$, onde cada condição foi deixada evoluir até o limite de 5 reflexões para a figura 2.13(a) e 100 reflexões para a figura 2.13(b). A evolução da dinâmica no tempo mostrado na figura 2.13 preenche o espaço de fases com um padrão de distribuição específico e as órbitas se aproximam primeiro da linha tracejada preta (que representa a condição de escape na superfície plana) ao invés da linha vermelha contínua (condição de escape na superfície corrugada). Os pontos verdes marcam as órbitas depois que o escape na região lisa ocorreu e caso considerássemos as fronteiras como espelhos. Se ambas as superfícies são espelhos, uma órbita iterada diversas vezes deve preencher totalmente o espaço caótico acessível e o padrão de distribuição dos pontos não seria observado. 


\section{GUIA DE ONDAS PERIODICAMENTE CORRUGADO}

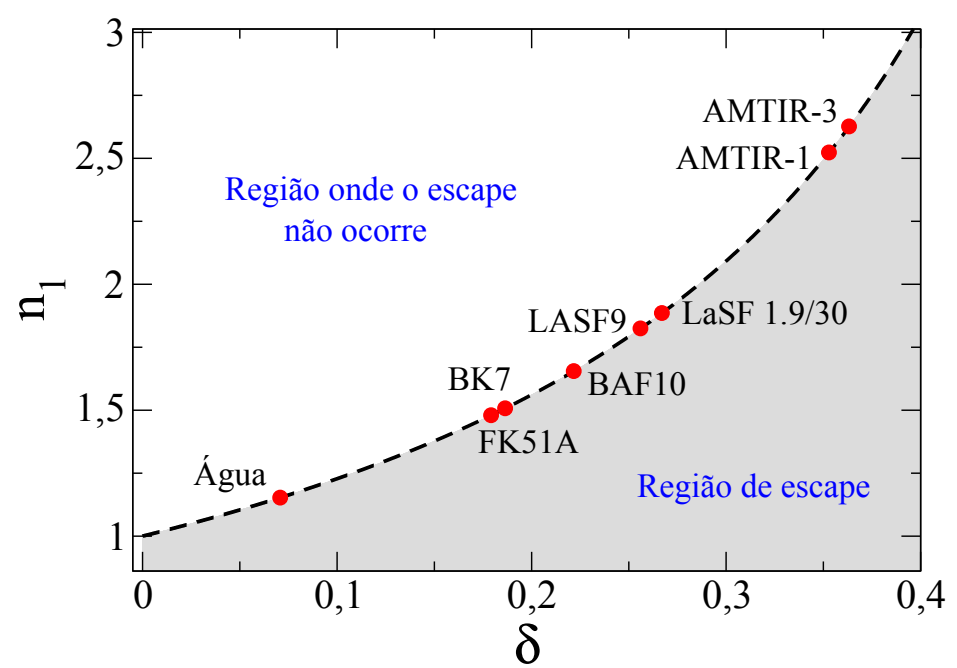

Figura 2.12: Índice de refração $\left(n_{1}\right)$ em função de $\delta$, onde destacamos alguns materiais. Os dados foram obtidos da Ref. (47).

Com a versão simplificada do mapa, poderemos dar uma descrição analítica e mais rigorosa do comportamento descrito pela Eq. (2.6). Para isto, consideraremos as seguintes hipóteses:

1. O valor do parâmetro $\delta$ é pequeno e; (a) a expressão do ângulo $\psi$ pode ser expandida em série de Taylor do tipo $\psi_{n+1}=\arctan \left(-\delta \sin \left(X_{n+1}\right)\right) \cong-\delta \sin \left(X_{n+1}\right)$; (b) e $2-\delta \cos \left(X_{n}\right)-\delta \cos \left(X_{n+1}\right) \cong 2$;

2. Consideraremos um novo ângulo chamado $\vartheta$ que é definido como $A_{n}=\pi / 2-\vartheta_{n}$, desta forma, próximo da curva invariante spanning teremos que este ângulo é bem pequeno. Como $\tan \left(A_{n}\right)=\tan \left(\pi / 2-\vartheta_{n}\right)$, após uma expansão em série de Taylor podemos concluir que $\tan \left(A_{n}\right) \cong 1 / \vartheta_{n}$.

Considerando este conjunto de hipóteses, o mapeamento que descreve as reflexões indiretas, aquelas que colidem com a parte corrugada vindo da superfície plana, é escrito como

$$
T:\left\{\begin{array}{l}
\vartheta_{n+1}=\vartheta_{n}-2 \delta \sin \left(X_{n+1}\right) \\
X_{n+1}=\left[X_{n}+\frac{2}{\vartheta_{n}}\right] \bmod (2 \pi)
\end{array} .\right.
$$

Este mapeamento é equivalente ao mapeamento do modelo Fermi-Ulam (3) em sua versão simplificada. Tal modelo considera uma partícula clássica sofrendo colisões entre duas paredes, sendo uma delas fixa e a outra se movendo como uma senóide. Como é uma versão simplificada, o mapeamento não traz as informações sobre colisões múltiplas com a parte corrugada do guia de ondas ou mesmo colisões múltiplas com a parede móvel do Fermi-Ulam. Vamos agora discutir uma propriedade existente no mar de caos do sistema. Consideramos que próximo da curva invariante spanning o ângulo pode ser escrito como

$$
\vartheta_{n}=\vartheta^{*}+(\Delta \vartheta)_{n}
$$



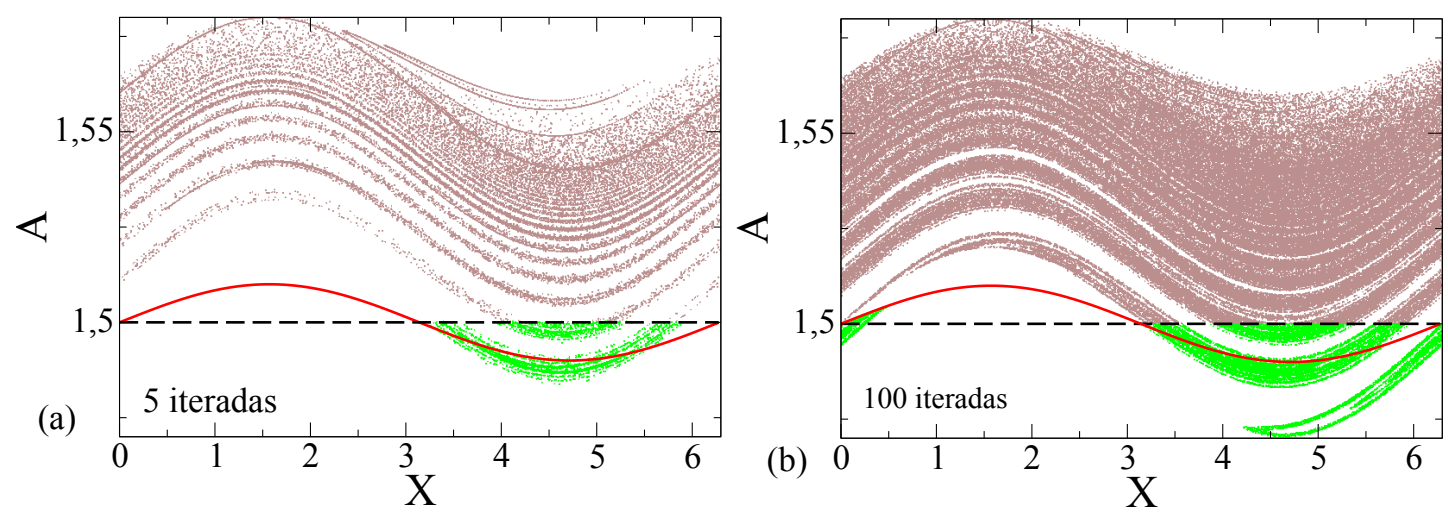

Figura 2.13: Evolução de $10^{4}$ condições iniciais diferentes iteradas: (a) 5 e; (b) 100 vezes. A curva preta tracejada representa o escape na região plana do bilhar enquanto a linha vermelha mostra o escape na parte corrugada do guia de ondas.

onde $\vartheta^{*}$ denota o ângulo médio ao longo da curva invariante spanning e $(\Delta \vartheta)_{n}$ corresponde a pequenas flutuações de $\vartheta^{*}$. A primeira equação de (2.10) é dada por

$$
(\Delta \vartheta)_{n+1}=(\Delta \vartheta)_{n}-2 \delta \sin \left(X_{n+1}\right) .
$$

A segunda equação de (2.10) é então escrita como $X_{n+1}=X_{n}+\frac{2}{\vartheta^{*}}\left(1+\frac{(\Delta \vartheta)_{n}}{\vartheta^{*}}\right)^{-1}$. Depois de expandir em série de Taylor e ignorar termos de ordem superior a 1, nós chegamos à

$$
X_{n+1}=X_{n}+\frac{2}{\vartheta^{*}}-\frac{2(\Delta \vartheta)_{n}}{\vartheta^{*^{2}}} .
$$

Após multiplicar a Eq. (2.12) por $\frac{-2}{\vartheta^{2}}$, adicionar o termo $\frac{2}{\vartheta^{*}}$, definir as variáveis $I_{n}=$ $\frac{2}{\vartheta^{*}}-\frac{2(\Delta \vartheta)_{n}}{\vartheta^{* 2}}$ e $\phi_{n}=X_{n}$ obtemos que

$$
\left\{\begin{array}{l}
I_{n+1}=I_{n}+\frac{4 \delta}{\vartheta^{* 2}} \sin \left(\phi_{n+1}\right) \\
\phi_{n+1}=\phi_{n}+I_{n} \bmod (2 \pi)
\end{array} .\right.
$$

O mapeamento (2.14) é conhecido como mapa padrão (standard mapping) (3) com um parâmetro de controle efetivo $K_{\text {eff }}=\frac{4 \delta}{\vartheta^{* 2}}$. O mapa padrão exibe uma transição de caos global para local em $K_{\text {eff }}=0,9716 \ldots$ (ver Ref. (3) para uma discussão mais específica). Isto é exatamente o que acontece na borda do mar caótico limitado pela curva invariante spanning. Se considerarmos $K_{\text {eff }}=0,9716 \ldots$, obtemos que o novo ângulo $\vartheta^{*}$ é dado por

$$
\vartheta^{*}=2 \sqrt{\frac{\delta}{0,9716 \ldots}} \cong 2,029 \delta^{1 / 2},
$$

que é muito próximo a expressão numérica obtida na Eq. (2.6). Nós então concluímos que o tamanho do mar caótico é proporcional ao parâmetro de controle $\sqrt{\delta}$. 


\section{GUIA DE ONDAS PERIODICAMENTE CORRUGADO}

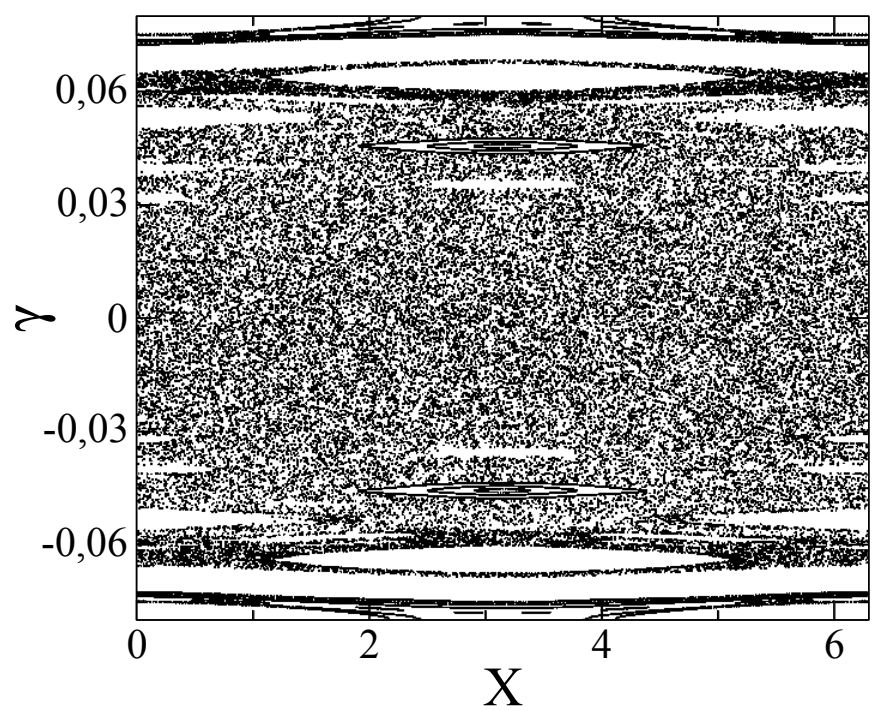

Figura 2.14: Espaço de fases para o mapeamento (2.16) usando o parâmetro de controle $\delta=10^{-3}$.

\subsection{Resultados numéricos - Versão simplificada do mapea- mento}

Nesta seção discutiremos os resultados para o guia de ondas em sua versão simplificada. Aqui apresentamos resultados do artigo citado na Ref. (29). Para escrever o mapeamento da mesma forma obtida no artigo citado, podemos fazer uma mudança de variável, por exemplo, considerando $\gamma_{n}=X_{n}+1 / \vartheta_{n}$. Somando $\left(1 / \vartheta_{n+1}\right)$ em ambos os lados da primeira equação do mapeamento (2.10) e considerando um atraso nas iteradas $(n \rightarrow n-1)$, é possível mostrar que

$$
T:\left\{\begin{array}{l}
X_{n+1}=\left[X_{n}+\left(\frac{1}{\gamma_{n}}+\frac{1}{\gamma_{n+1}}\right)\right] \bmod (2 \pi) \\
\gamma_{n+1}=\gamma_{n}+2 \delta \sin \left(X_{n}+\frac{1}{\gamma_{n}}\right)
\end{array} .\right.
$$

O determinante da matriz Jacobiana é igual a 1 e com isso, podemos afirmar que o mapa preserva a área no espaço de fases.

A figura 2.14 mostra o espaço de fases gerado por iterações do mapeamento (2.16). Podemos identificar algumas estruturas, como curvas invariantes do tipo spanning e ilhas periódicas. O mar caótico foi caracterizado usando expoentes de Lyapunov. O valor médio do expoente de Lyapunov positivo encontrado para o parâmetro de controle $\delta=10^{-3}$ foi $\bar{\lambda}=1,624(7)$, onde o erro 0,007 corresponde ao desvio padrão do conjunto de 10 diferentes condições iniciais escolhidas ao longo do mar caótico. O comportamento do expoente de Lyapunov positivo como função do parâmetro $\delta$ é mostrado na figura 2.15 .

Podemos ver que a variação do expoente de Lyapunov é pequena, da ordem de $10 \%$ para a variação de $\delta$ considerada $\left(\delta \in\left[10^{-5}, 10^{-2}\right]\right)$. 


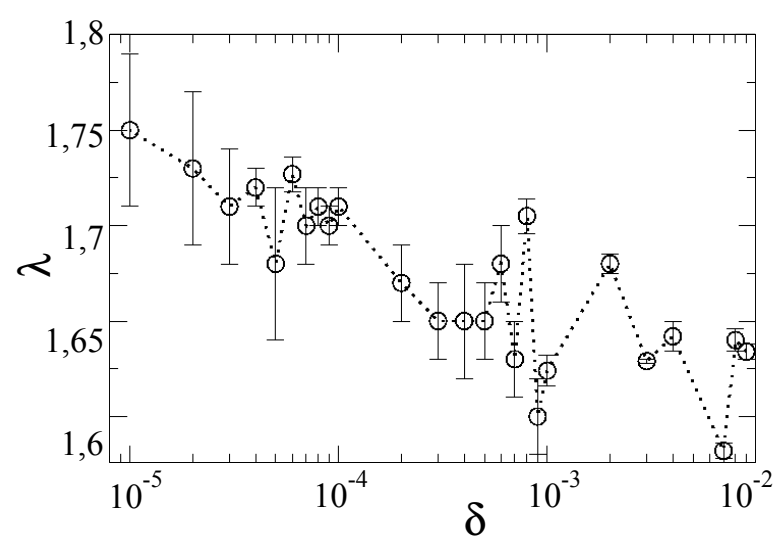

Figura 2.15: Expoente de Lyapunov positivo como função do parâmetro de controle $\delta$.

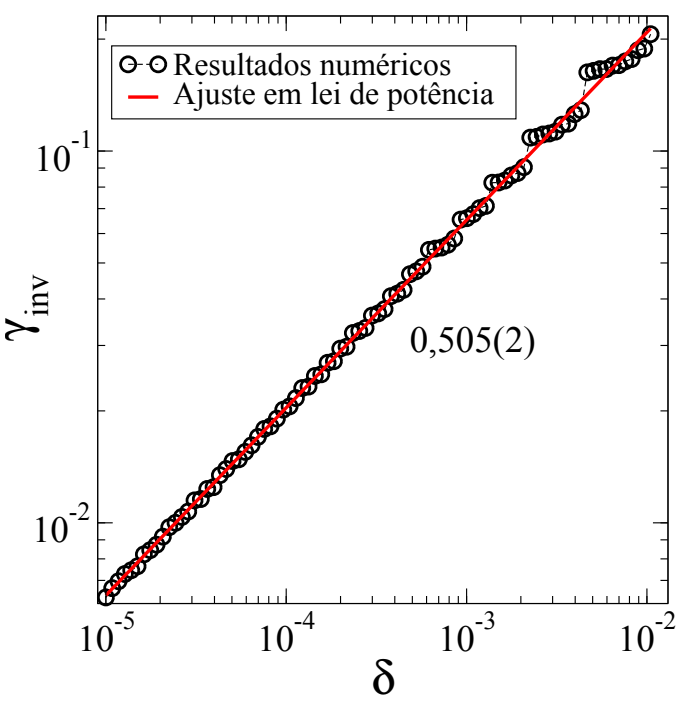

Figura 2.16: Gráfico do menor valor de $\gamma$ na curva invariante spanning como função de $\delta$ junto com o resultado mostrado na Eq. (2.19). Um ajuste em lei de potência nos fornece $\gamma \propto \delta^{0,505(2)}$.

Como o parâmetro de controle $\delta$ varia, a posição das duas curvas invariantes spanning (positiva e negativa) também varia. Uma vez que essas duas curvas separam o caos global do caos local, nós esperamos que a dinâmica do sistema possa ser descrita localmente usando o mapa padrão. Para isso, usaremos um procedimento similar ao usado na Ref. (48) e escreveremos o ângulo de reflexão como

$$
\gamma_{n+1} \cong \gamma^{*}+\Delta \gamma_{n+1},
$$

onde $\gamma^{*}$ é um valor típico do ângulo de reflexão ao longo da curva invariante spanning e $\Delta \gamma_{n+1}$ é uma pequena perturbação deste ângulo. Se considerarmos $Z_{n}=X_{n}+1 / \gamma_{n}$, reescrevermos a primeira equação do mapeamento (2.16), expandirmos em série de Taylor, rearranjarmos o resultado e definirmos as novas variáveis $I_{n+1}=-\frac{2 \Delta \gamma_{n+1}}{\gamma^{* 2}}+\frac{2}{\gamma^{*}}$ e $\phi_{n}=Z_{n}+\pi$, nós obteremos

$$
T:\left\{\begin{array}{l}
I_{n+1}=I_{n}+\frac{4 \delta}{\gamma^{* 2}} \sin \left(\phi_{n}\right) \\
\phi_{n+1}=\phi_{n}+I_{n+1}
\end{array},\right.
$$

onde podemos identificar um parâmetro de controle efetivo dado por $K_{\mathrm{eff}}=4 \delta / \gamma^{* 2}$. A transição de caos global para local ocorre para $K_{\text {eff }} \cong 0,971 \ldots$, com isso, obtemos que as duas curvas invariantes spanning que separam o mar caótico são dadas por

$$
\gamma^{*} \cong \pm 2 \sqrt{\frac{\delta}{0,971 \ldots}} .
$$




\section{GUIA DE ONDAS PERIODICAMENTE CORRUGADO}
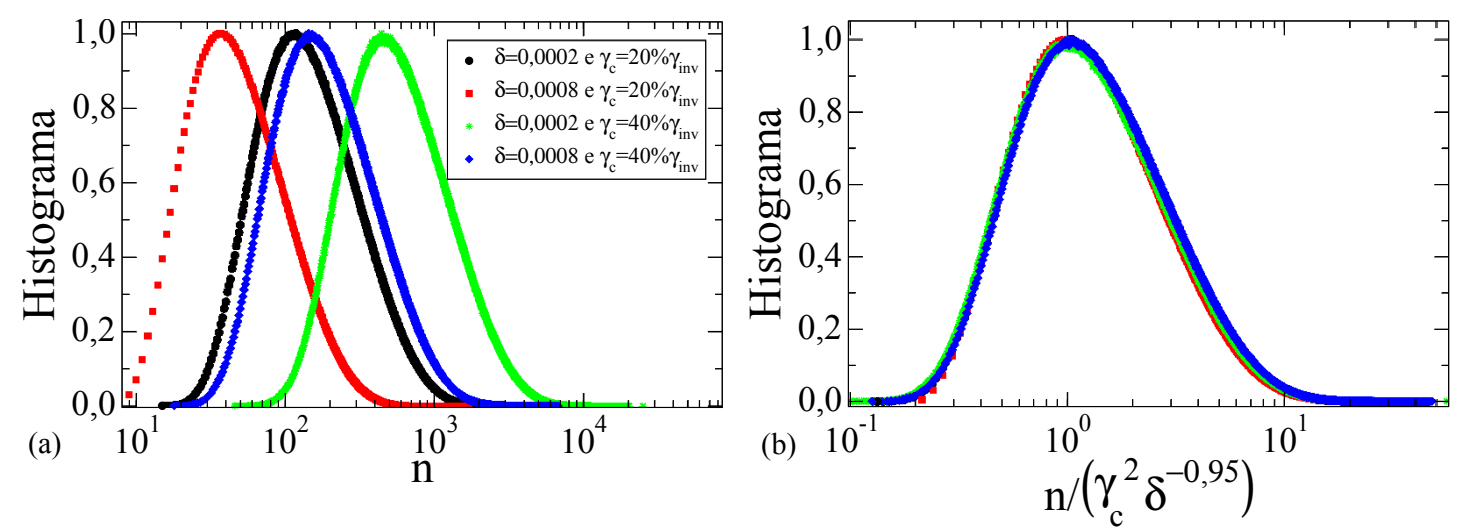

Figura 2.17: (a) Histograma para diferentes $\gamma_{c}$ e diferentes valores de $\delta$. (b) Colapso de todas as curvas mostradas no item (a) em um único e universal gráfico após uma reescala $n \rightarrow n /\left(\gamma_{c}^{u} \delta^{\bar{\zeta}}\right)$

Com isso, podemos concluir que o tamanho do mar caótico é proporcional à $\sqrt{\delta}$ como discutido na Ref. (48). A figura 2.16 confirma, a partir de simulações numéricas da posição da curva invariante, o resultado previsto pela Eq. (2.19).

Agora estudaremos o transporte de partículas através do mar caótico. Como condições iniciais consideraremos $\gamma_{0}=10^{-5} \delta$ e o valor de $X_{0}$ será aleatório. Permitiremos que a dinâmica possa evoluir até $10^{7}$ colisões usando um conjunto de $10^{9}$ condições iniciais diferentes com $X_{0} \in[0,2 \pi)$. Durante a evolução da dinâmica se $\left|\gamma_{n}\right| \geq \gamma_{c}$ iremos interromper a simulação, onde coletaremos o número da colisão e então uma nova condição inicial será escolhida. Usando tal procedimento obteremos o histograma do número de partículas que atingiram $\gamma_{c}$ em uma certa iterada $n$, como mostrado na figura 2.17(a) para diferentes valores do parâmetro de controle e para diferentes valores de $\gamma_{c}$. Podemos ver um rápido crescimento para pequenos valores de $n$, até o histograma atingir um valor máximo em $n_{p}$. Após isso, existe um decaimento para grandes valores de $n$, que se aproxima de zero assimptoticamente. Para o conjunto usado, poucas órbitas sobrevivem até $10^{7}$ iteradas. Entretanto, o histograma mostrado na figura 2.17(a) foi reescalado pelo máximo $10^{5}$ apenas por caráter visual. A posição do pico $n_{p}$ é proporcional à $\gamma_{c}$. Um gráfico de $n_{p} v s \gamma_{c}$ nos fornece um comportamento descrito por lei de potência com expoente $u=1,97(1)$, como é mostrado na figura $2.18(\mathrm{a})$.

Se considerarmos o transporte no sistema como uma difusão Browniana normal, nós deveríamos esperar que $n_{p}$ seja proporcional à $\gamma_{c}^{2}$. Entretanto, devido ao stickiness e aprisionamento de partículas perto das ilhas, o expoente obtido é um pouco menor que 2. De fato, nossas simulações forneceram o valor 1,97(1), como mostrado na figura 2.18(a). O gráfico de $n_{p} v s \delta$ para diferentes valores de $\gamma_{c}$ é mostrado na figura 2.18(b) para os seguintes parâmetros, junto com os expoentes $\zeta$ obtidos após ajustes em lei de potência: $\gamma_{c}=20 \% \gamma_{i n v} \operatorname{com} \zeta=-0,950(8) ; \gamma_{c}=40 \% \gamma_{i n v}$ e $\zeta=-0,951(6)$; finalmente $\gamma_{c}=70 \% \gamma_{i n v} \operatorname{com} \zeta=-0,97(2)$. O valor médio é $\bar{\zeta}=-0,95(1)$. Com este resultado, o 

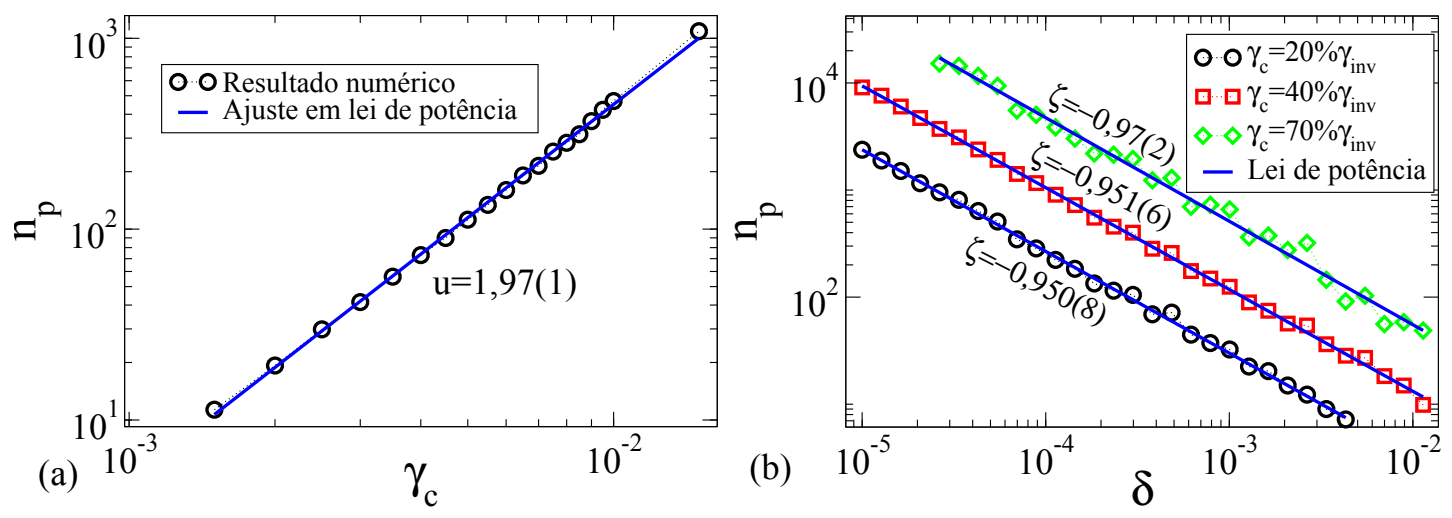

Figura 2.18: (a) Gráfico de $n_{p}$ vs $\gamma_{c}$. Após um ajuste em lei de potência o expoente obtido foi $u=1,97(1)$. (b) Gráfico de $n_{p} \times \delta$ e os respectivos ajustes em lei de potência para $\gamma_{c}=20 \% \gamma_{i n v} \operatorname{com} \zeta=-0,950(8) ; \gamma_{c}=40 \% \gamma_{i n v}$ com $\zeta=-0,951(6)$; e finalmente $\gamma_{c}=70 \% \gamma_{i n v} \operatorname{com} \zeta=-0,97(2)$.

histograma pode ser reescalado convenientemente afim de colapsar todas as curvas em uma única curva universal, como é mostrado na figura 2.17(b), com isso, confirmando uma invariância de escala.

\subsection{Conclusões}

Como conclusão, nós consideramos o problema de um raio de luz refletindo em um guia de ondas periodicamente corrugado. Mostramos com detalhes a forma de se obter os mapeamentos, tanto para a versão completa ou simplificada. O espaço de fases obtido é do tipo misto, contendo ilhas periódicas, caos e curvas invariantes spanning. Para a versão completa do mapeamento, mostramos que o histograma do número de reflexões múltiplas no espelho corrugado é invariante de escala com relação ao parâmetro de controle. O desvio do ângulo médio foi analisado e mostramos que também é invariante de escala. Consideramos as equações de Fresnel para determinar em quais situações um raio de luz seria refratado ou refletido e comparamos com diversos materiais que possuem diferentes índices de refração. Para a versão simplificada do problema, o mar caótico confinado entre as duas primeiras curvas invariantes spanning (positiva e negativa) foi caracterizado usando argumentos de escala juntamente com uma conexão com o mapa padrão. Quando uma janela de escape é introduzida no mar caótico, o histograma de escape exibe um crescimento marcado por um máximo e então decaindo para longas iteradas. O máximo do histograma foi usado para reescalar todas as curvas de histograma em uma única curva universal, ambos obtidos em função do parâmetro de corrugação e também posição do escape. Seria interessante no futuro considerarmos as escalas para o caso quântico. Os resultados apresentados aqui foram recentemente publicados no Journal of Physics A, Mathematical and Theoretical (28), Physics Letters A (29) e Communications in Nonlinear Science $\&$ Numerical Simulation (30). 
2. GUIA DE ONDAS PERIODICAMENTE CORRUGADO 
$\Gamma_{\text {Capítulo }} 3$

\section{Invariância de escala do coeficiente de difusão em uma família de mapeamentos Hamiltonianos bidimensionais}

\subsection{Resumo}

Consideramos uma família de mapeamentos bidimensionais que preservam a área no espaço de fases. Esta pode ser considerada uma generalização do mapa padrão de Chirikov. Estes modelam uma grande variedade de sistemas periodicamente forçados. A variável ação difunde com incrementos cuja fase é controlada por uma potência negativa da ação e, portanto, efetivamente não correlacionadas para pequenas ações. Para valores maiores da ação o espaço de fases é misto. O transporte de partículas ao longo do espaço de fases é considerado iniciando um conjunto de partículas com valores de ação muito pequenos e então evoluímos estas no espaço de fases até que elas atinjam uma certa altura $h$. Para órbitas caóticas abaixo das ilhas periódicas, a probabilidade de sobrevivência é caracterizada por uma função exponencial, que é modelada pela solução da equação de difusão. Por outro lado, quando $h$ atinge a posição das ilhas periódicas, a difusão diminui significativamente. Mostramos que o coeficiente de difusão é invariante de escala em relação aos parâmetros de controle do mapeamento. Este capítulo apresenta os resultados publicados na revista Physical Review E (31).

\subsection{Motivação}

A caracterização de espaços de fases mistos regulares-caóticos continua sendo um dos problemas mais desafiadores em dinâmica Hamiltoniana. Quando temos uma força periódica agindo, o nível de caoticidade é tipicamente controlado pela razão das escalas temporais intrínsecas e extrínsecas, uma função das variáveis do espaço de fases. Por 


\section{INVARIÂNCIA DE ESCALA DO COEFICIENTE DE DIFUSÃO EM UMA FAMÍLIA DE MAPEAMENTOS HAMILTONIANOS BIDIMENSIONAIS}

exemplo, a família de mapas

$$
T:\left\{\begin{array}{l}
J_{n+1}=\left|J_{n}-\epsilon \sin \left(2 \pi \theta_{n}\right)\right| \\
\theta_{n+1}=\left[\theta_{n}+J_{n+1}^{-\gamma}\right] \quad(\bmod 1)
\end{array},\right.
$$

para ação $J$ e ângulo $\theta$ descreve uma vasta classe de sistemas, incluindo aplicações relevantes para física de plasmas $\operatorname{com} \gamma=2$ (49). Para $\gamma=3 / 2$, a difusão de parâmetros orbitais de cometas através do movimento periódico de Júpiter é obtido (50, 51). Para este problema a variável ação corresponde à energia dos cometas e a segunda equação recupera a terceira lei de Kepler, devido a este fato este é chamado de mapa de Kepler. $\mathrm{O}$ valor de $\gamma=1$ recupera a dinâmica de um pacote de ondas (49). Para $\gamma=1$ temos também uma partícula se movendo entre uma placa vibratória e outra fixa (52). Este caso recupera o modelo do acelerador de Fermi-Ulam unidimensional, o qual considera uma partícula sofrendo colisões elásticas entre duas paredes rígidas. A variável ação representa a velocidade da partícula e o ângulo denota a fase da parede móvel. $\gamma=1$ também recupera um guia de ondas periodicamente corrugado (53), conforme discutido no capítulo anterior. Aqui a variável ação corresponde à posição no ponto de reflexão, enquanto a variável ângulo corresponde ao ângulo formado entre a trajetória da luz e a reflexão com a superfície corrugada. Já para $\gamma=1 / 2$, algumas propriedades dinâmicas para um poço de potencial $(54,55)$ são recuperados. Este modelo consiste de uma partícula clássica confinada dentro de uma caixa com potenciais infinitos nas bordas e que contém um poço de potencial oscilante no meio. Assume-se que o fundo do potencial se move periodicamente no tempo de acordo com uma função cosseno. A variável ação representa a energia da partícula enquanto a ângulo corresponde a fase de oscilação. Para $\gamma=-1$ o modelo para uma partícula saltando sobre uma placa vibratória é recuperado (56). O modelo descreve o problema de uma partícula clássica colidindo elasticamente com uma parede cuja posição varia periodicamente no tempo, onde a partícula sofre a influência de um campo gravitacional. $\gamma=-1$ recupera também o mapa padrão de Chirikov (3). Tal modelo descreve um sistema perturbado por uma sequência de pulsos. Para maior clareza, vamos a partir de agora assumir que $\gamma>0$. A separação de escala de tempo é dada por $J^{-\gamma}$. Quando $J \approx 1$ existe um espaço de fases misto, enquanto $J \ll 1, \theta$ é efetivamente aleatório, levando à uma difusão caótica de $J$ o qual pode ser descrita analiticamente. Aqui desenvolvemos uma abordagem de sistema aberto para regiões caótica e mista, e demonstramos invariância de escala do coeficiente de difusão em relação ao parâmetro de controle $\epsilon$.

Uma técnica para analisar sistemas dinâmicos $(57,58)$ consiste em colocar um "buraco" quando $J>h$, onde $h$ é um parâmetro que define a posição do buraco. Podemos definir a probabilidade de sobrevivência $P(n)$ como o número de partículas que sobrevivem (ou seja, a variável ação é menor do que $h$ ) para uma dada colisão $n$. Isto nos fornece um teste sensível da difusão da variável ação. No regime fortemente caótico, $P(n) \sim e^{-A n}(13,32)$ com uma taxa de escape $A$ que pode ser previsto pela solução da equação de difusão com apropriadas condições iniciais e de contorno e sem parâmetros livres. A relação dos coeficientes de transporte (por exemplo, difusão) e da taxa de escape $A$ em sistemas moleculares foi introduzido como "o formalismo da taxa 
de escape" $(59,60)$. Aqui a difusão é no espaço de fases e mais adiante nós prevemos a completa dependência temporal $P(n)$.

Com o aumento da variável ação, movimentos periódicos são observados levando a existência de ilhas periódicas. Para valores ainda maiores da ação um conjunto de curvas invariantes spanning está presente no espaço de fases limitando o crescimento da variável ação. Devido a existência de ilhas periódicas, as órbitas podem ficar aprisionadas ao redor dessas regiões periódicas. Este aprisionamento temporário, também chamado de stickiness, afeta o coeficiente de difusão, que é sensível à posição de ilhas periódicas. Um observável relevante para sabermos o início do stickiness é a localização da ilha periódica mais baixa $J_{L S I}$. Neste trabalho mostramos que o processo de difusão é invariante de escala e o coeficiente de difusão reescalado $D / \epsilon^{2}$, calculado usando uma abordagem de sistema aberto, é uma função universal da altura do buraco reescalada $h / J_{L S I}$.

Este capítulo é organizado da seguinte forma: Na seção 3.3 estudamos uma abordagem analítica para os coeficientes de difusão e estudamos as propriedades de transporte. A seção 3.4 discute sobre propriedades de transporte e resultados. Por último, na seção 3.5 mostramos as discussões finais.

\subsection{Coeficiente de difusão}

Consideramos agora a dinâmica no espaço de fases para pequenos valores de $J$. Devido a falta de correlação entre a variável ângulo nos instantes $n$ e $n+1$, os incrementos em $J$ são bem descritos usando o Teorema do Limite Central (TLC) (61, 62). Podemos então dizer que ao longo de muitos passos de tempo, a distribuição de deslocamentos é Gaussiana com uma variância proporcional ao número de passos. Condições suficientes para termos um TLC são que $\left\{X_{n}\right\}_{n=1}^{\infty}$ é uma sequência de variáveis aleatórias Independentes e Identicamente Distribuídos (IID) com valores reais tal que: (i) a média $\left\langle X_{n}\right\rangle=0$ e; (ii) $\left\langle X_{n}^{2}\right\rangle<\infty(63,64,65)$. Na Eq. (3.1) vemos que os ângulos divergem no limite em que ação tende a zero, então espera-se que estes sejam independentes e distribuídos uniformemente no intervalo $[0,1)$. O incremento de $J$ na Eq. (3.1) e também em muitos outros problemas físicos da literatura (66) é dado por $\Delta_{n}=J_{n+1}-J_{n}=-\epsilon \sin \left(2 \pi \theta_{n}\right)$, e este é também IID. Então, é possível observar que $\left\langle\Delta_{n}^{2}\right\rangle=\int_{0}^{1} \epsilon^{2} \sin ^{2}(2 \pi \theta) d \theta=\epsilon^{2} / 2$. Portanto o coeficiente de difusão analítico é dado por $D=\left\langle\Delta^{2}\right\rangle / 2=\epsilon^{2} / 4$, e por isso, $D / \epsilon^{2}=1 / 4$. Ignoramos os sinais $J<\epsilon$; o efeito causado é para assegurar que $J$ permanece positivo, i.e. existe uma parede rígida no processo de difusão em $J=0$.

Após muitas iteradas, devemos considerar o processo contínuo dado pela equação de difusão

$$
\frac{\partial u}{\partial n}=D \frac{\partial^{2} u}{\partial J^{2}},
$$

onde $u$ é uma função de densidade de probabilidade. As condições de contorno são dadas por $d u / d J(0, n)=0$ (a parede rígida, acima) e $u(h, n)=0$ (escape através do buraco). Este problema deve ser resolvido através de separação de variáveis, $u(J, n)=$ $X(J) T(n)$, onde as condições de contorno fornecem $X(J)=\cos [J \pi(k+1 / 2) / h]$, com 


\section{INVARIÂNCIA DE ESCALA DO COEFICIENTE DE DIFUSÃO EM UMA FAMÍLIA DE MAPEAMENTOS HAMILTONIANOS BIDIMENSIONAIS}

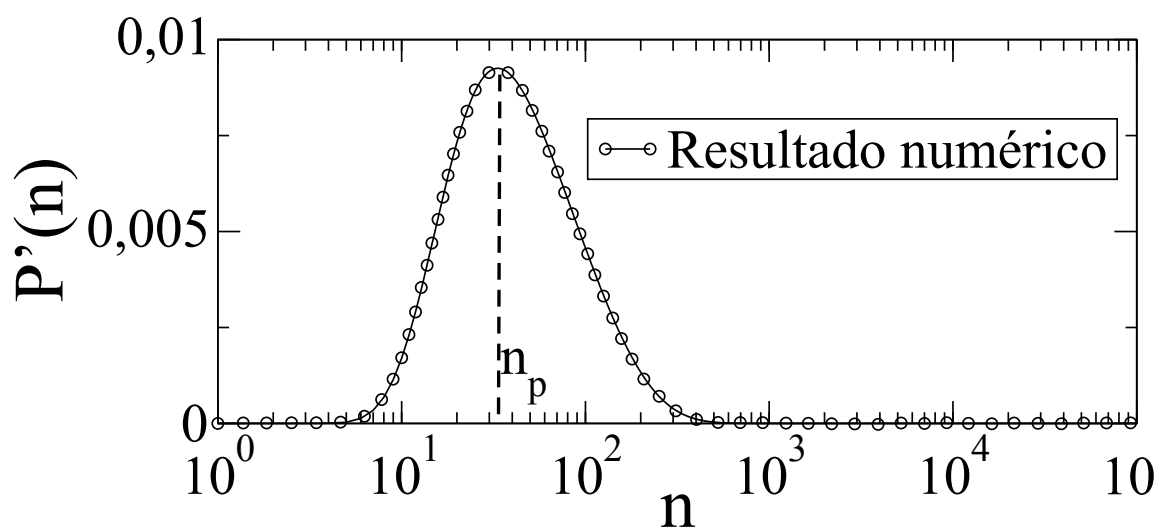

Figura 3.1: Gráfico de $P^{\prime}$ vs. $n$ considerando $h=10$ e $D=0,5$.

$k=0,1,2,3, \ldots$ Substituindo $u(J)$ na Eq. (3.2), obtemos a solução da equação de difusão,

$$
u(J, n)=\sum_{k=0}^{\infty} a_{k} \cos \left[\frac{J \pi\left(k+\frac{1}{2}\right)}{h}\right] \exp \left[\frac{-D n \pi^{2}\left(k+\frac{1}{2}\right)^{2}}{h^{2}}\right] .
$$

Finalmente, os coeficientes desconhecidos $a_{k}$ são determinados pelas condições iniciais. Iniciamos todas as partículas perto de $J=0$, então $u(J, 0)=\delta(J)$. Equacionando esta com $a_{k} \cos [J \pi(k+1 / 2) / h]$ como pedido pela Eq. (3.3), multiplicando ambos os lados por $\cos [J \pi(m+1 / 2) / h]$ e integrando em função de $J$ nos limites $[0, h]$ encontramos

$$
u(J, n)=\frac{2}{h} \sum_{k=0}^{\infty} \cos \left[\frac{J \pi\left(k+\frac{1}{2}\right)}{h}\right] \exp \left[\frac{-D n \pi^{2}\left(k+\frac{1}{2}\right)^{2}}{h^{2}}\right],
$$

o qual satisfaz a Eq. (3.2), ambas as condições de contorno e também as condições iniciais. Integrando a Eq. (3.4) em função de $J$ no intervalo $[0, h]$, obtemos a probabilidade de sobrevivência, que é dada por

$$
P(n)=\frac{2}{\pi} \sum_{k=0}^{\infty} \frac{\sin \left[\pi\left(k+\frac{1}{2}\right)\right]}{k+\frac{1}{2}} \exp \left[\frac{-D n \pi^{2}\left(k+\frac{1}{2}\right)^{2}}{h^{2}}\right] .
$$

O negativo da derivada de $P(n)$ em relação a $n$, i.e., $P^{\prime}(n)=-d P(n) / d n$, nos fornece o histograma de frequência para o número de partículas que escaparam em um tempo $n$. Um gráfico de $P^{\prime}(n)$ vs. $n$ é mostrado na figura 3.1.

Vemos a partir de tal figura que para curtos $n$ existe um regime de crescimento do histograma de frequência. Este atinge um máximo em $n_{p}$ e então decresce para altos valores de $n$. Poucas partículas podem viajar até $h$ e então escapar para curtos tempos. O pico significa que para um valor típico de $n$ existe um grupo majoritário de partículas que escapam atingindo $h$. Para tempos longos existem apenas poucas partículas restando, e então o número de escapes diminui. Considerando a figura 3.1 juntamente com a expressão de $P^{\prime}(n)$, vemos que para elevados valores de $h$ um único 


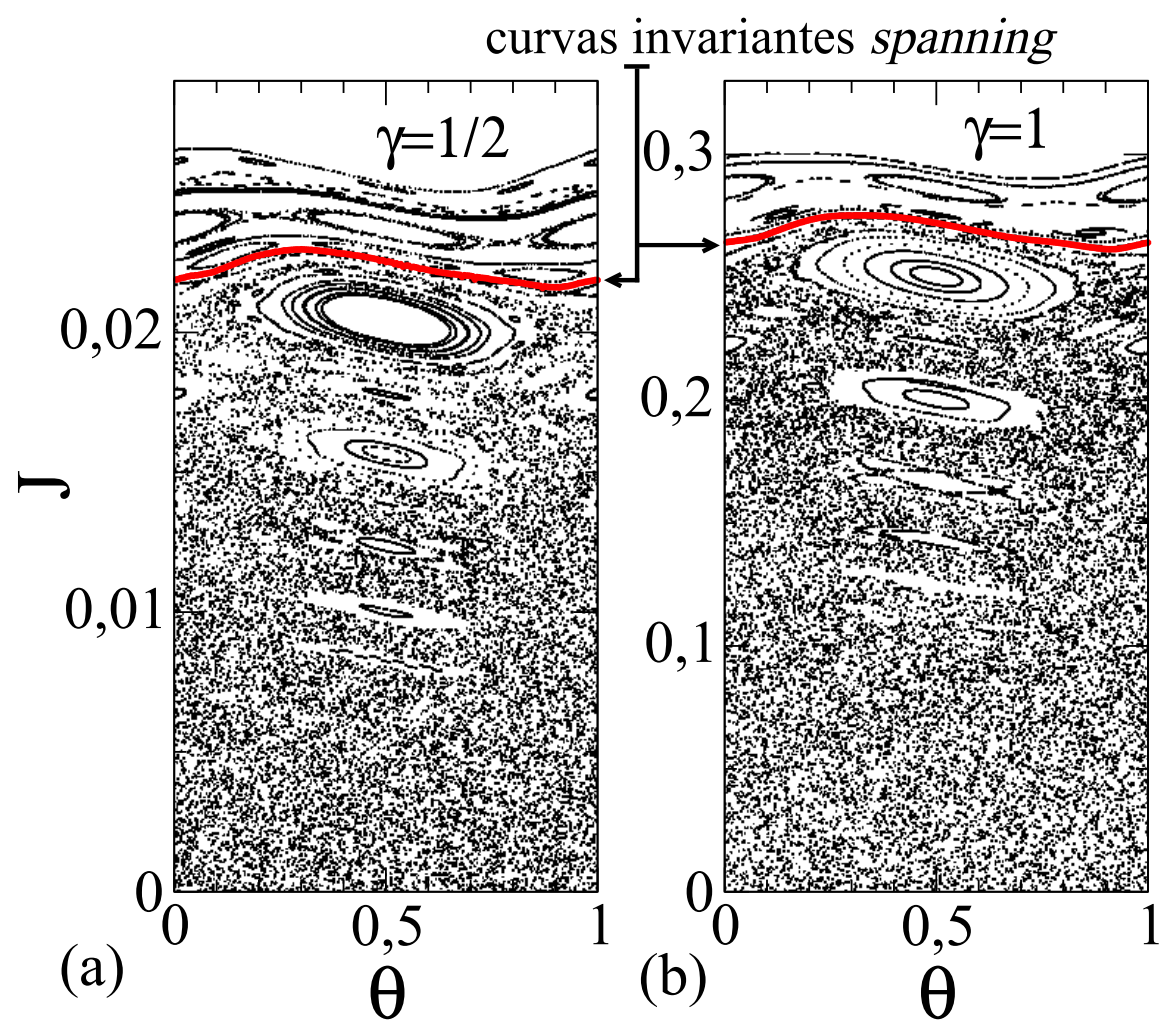

Figura 3.2: Espaço de fases usando: (a) $\epsilon=10^{-3}$ e $\gamma=1 / 2$, (b) $\epsilon=10^{-2}$ e $\gamma=1$.

termo com $k=0$ é dominante e então $\exp \left[-D n \pi^{2} /\left(4 h^{2}\right)\right] \cong \exp [-A n]$. Desta forma, o coeficiente de difusão é escrito como

$$
D=4 h^{2} A / \pi^{2} .
$$

O coeficiente $A$ pode ser obtido utilizando um ajuste exponencial no histograma de frequência $P^{\prime}(n)$ vs. $n$ para a curva que está decaindo depois do pico e antes do final da curva (que pode não ser exponencial para tempos muito longos devido ao stickiness).

\subsection{Propriedades de transporte e resultados}

Os resultados acima são bastante gerais, em princípio, podendo ser aplicados para qualquer sistema modelado pela equação de difusão. Agora consideraremos especificamente uma família de mapeamentos dada pela Eq. (3.1). Os parâmetros de controle são $\epsilon$ e $\gamma$. Para $\epsilon=0$ o sistema é integrável enquanto é misto para qualquer $\epsilon \neq 0$. O mapeamento preserva a área no espaço de fases uma vez que o determinante da matriz Jacobiana é igual à 1. A relevância dos valores particulares de $\gamma$ é mostrada na seção de motivação. O espaço de fases gerado pelo mapeamento (3.1) é mostrado na figura 3.2 para os parâmetros de controle: (a) $\epsilon=10^{-3}$ e $\gamma=1 / 2$ e (b) $\epsilon=10^{-2}$ e $\gamma=1$. Vemos 


\section{INVARIÂNCIA DE ESCALA DO COEFICIENTE DE DIFUSÃO EM UMA FAMÍLIA DE MAPEAMENTOS HAMILTONIANOS
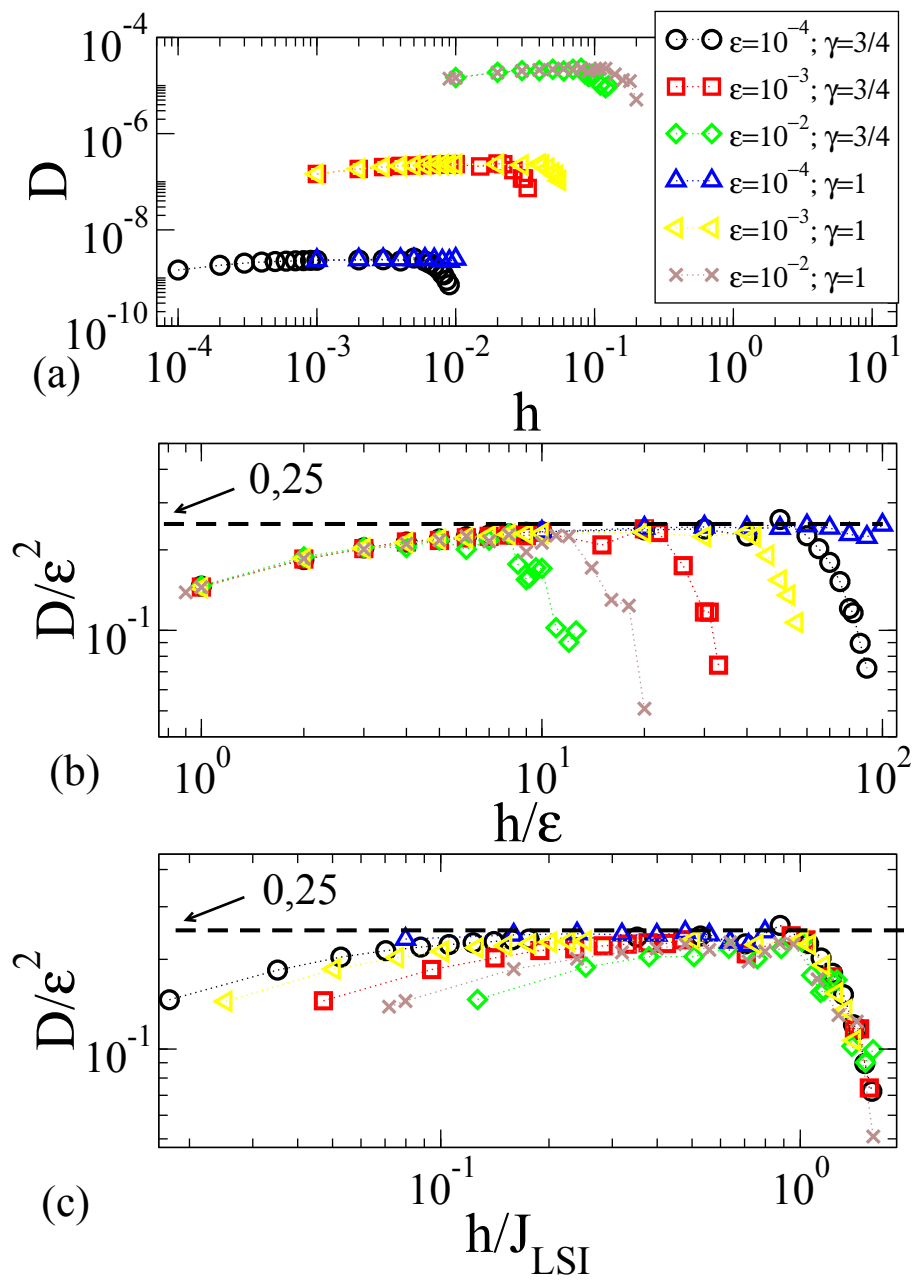

Figura 3.3: (a) D vs. $h$ para diferentes valores de $\epsilon$ e $\gamma$. (b) Depois de uma reescala nos eixos, as curvas colapsaram para pequenos valores de $h$. (c) Depois de uma reescala apropriada nos eixos, todas as curvas colapsaram para altos valores de $h$.

que o espaço de fases é misto, com caos para pequenos valores de $J$, um conjunto de ilhas periódicas que são rodeadas por um mar caótico, e este último é limitado por um conjunto de curvas invariantes spanning.

O tamanho do mar caótico pode ser estimado usando a posição da curva invariante spanning mais baixa. Como discutido em (67), perto de tal curva, a ação pode ser aproximada como $J_{n+1} \cong J^{*}+\Delta J_{n+1}$ onde $J^{*}$ é um valor típico ao longo da curva e $\Delta J_{n+1}$ é uma pequena perturbação de $J^{*}$. Usando tal procedimento e fazendo uma conexão com o mapa padrão de Chirikov (3), o qual possui uma transição de caos global para local em $K=0,9716 \ldots$, a primeira curva invariante spanning pode ser descrita como $J^{*} \cong\left[\frac{2 \pi \gamma \epsilon}{0,9716 \ldots}\right]^{1 /(1+\gamma)}$. A equação de difusão dada por $(3.2)$ e o coeficiente de difusão obtido na Eq. (3.6) podem ser aplicados também para a região caótica do 

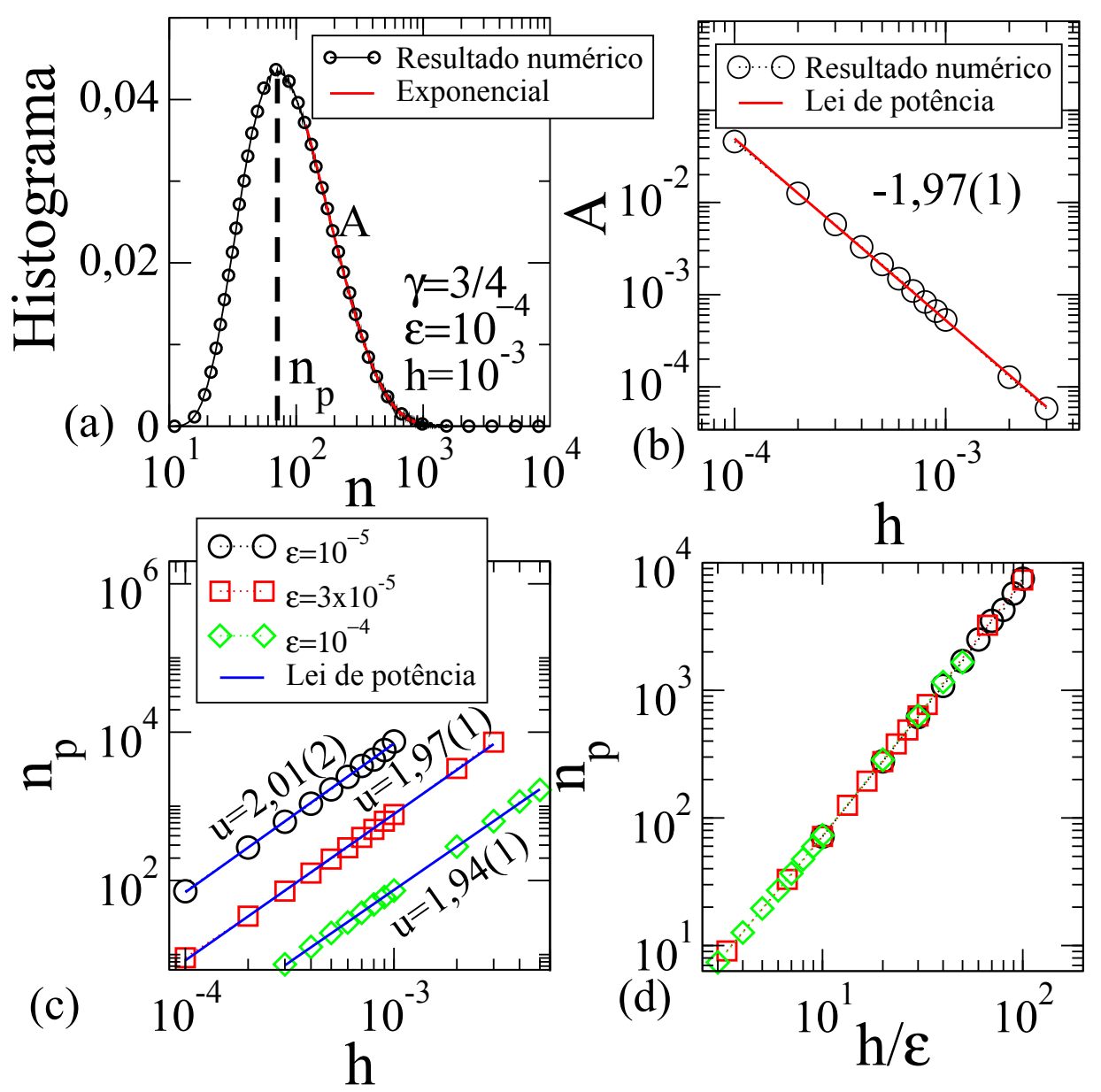

Figura 3.4: Para $\gamma=3 / 4$ temos: (a) Um gráfico do Histograma em função de $n_{p}$, onde destacamos como encontrar $n_{p}$; (b) $A$ vs. $h$, onde a inclinação é igual à $-1,97(1)$; (c) $n_{p}$ vs. $h$ para diferentes valores de $\epsilon: \epsilon=10^{-5}, \epsilon=3 \times 10^{-5}$ e $\epsilon=10^{-4}$ e (d) $n_{p} v s . h / \epsilon$.

espaço de fases gerado pelo mapeamento (3.1) abaixo da ilha periódica com menor valor da ação. Quando as ilhas de estabilidade são consideradas, um aprisionamento local pode ser observado. Este regime de stickiness afeta diretamente o transporte das partículas no espaço de fases levando à uma redução do coeficiente de difusão $D$ (como podemos ver na figura 3.3). O entendimento dos efeitos de stickiness no transporte é ainda um dos problemas em aberto na dinâmica não linear.

As condições de contorno no espaço de fases são definidas pelo menor valor da ação (por exemplo, zero) e a posição $h<J^{*}$ que define a localização do buraco. Para tomar uma média das quantidades, começamos com um conjunto de diferentes condições iniciais com valor de ação pequeno $\left(10^{-3} \epsilon\right)$ e diferentes ângulos $\theta_{0}$ uniformemente distribuídos ao longo de $\theta_{0} \in[0,1]$. Se durante a dinâmica a partícula atinge $h$, a simulação será interrompida, o número de iteradas necessário para difusão da partícula até $h$ é coletado e uma nova condição inicial é tomada com um ângulo inicial diferente. O 


\section{INVARIÂNCIA DE ESCALA DO COEFICIENTE DE DIFUSÃO EM UMA FAMÍLIA DE MAPEAMENTOS HAMILTONIANOS BIDIMENSIONAIS}

processo é repetido até que todo o conjunto de condições iniciais seja considerado. Se o tempo máximo permitido de $n=10^{5}$ iteradas é atingido, consideramos que a partícula não escapa (poderia escapar para tempos maiores e para uma simulação maior) e uma nova condição inicial é iniciada.

É conhecido que quando o escape é considerado em uma região onde apenas caos é presente, a probabilidade de sobrevivência de um conjunto de partículas movendo em tal região é descrito por um decaimento exponencial (ver, por exemplo, $(68,69)$ ). A existência de regiões periódicas no espaço de fases incluindo ilhas de estabilidade e curvas invariantes levam a um aprisionamento local devido ao stickiness (70, 71, 72) e consequentemente a uma difusão anômala (73) transformando o decaimento da probabilidade de sobrevivência em um regime mais lento que pode incluir desde uma lei de potência $(74,75)$ ou até mesmo uma stretched exponential (16). Em nossas simulações, a posição $h$ foi variada. Para valores de $h$ abaixo das ilhas de estabilidade, um decaimento exponencial descreve bem a probabilidade de sobrevivência, enquanto um decaimento mais lento é observado quando ilhas periódicas estão presentes. A figura 3.4(a) mostra um gráfico do histograma de frequência considerando os parâmetros $\gamma=3 / 4$ e $\epsilon=10^{-4}$. Entretanto qualquer outro conjunto de parâmetros geraria um gráfico semelhante, portanto o comportamento parece ser genérico para qualquer $\gamma>0$ e $\epsilon \neq 0$. O parâmetro $A$ usado na Eq. (3.6) é obtido por uma análise numérica do decaimento da curva depois de atingir o pico em $n_{p}$ e antes do final da curva (onde flutuação devido ao tamanho do conjunto deve influenciar). A figura 3.4(b) mostra um gráfico de $A$ vs. $h$ e um ajuste em lei de potência nos fornece como inclinação o valor $-1,97(1) \cong-2$, que concorda com a Eq. (3.6). O pico do histograma é sensível a posição de $h$. Este se move para a direita na figura 3.4(a) quando a posição de $h$ é aumentada na direção vertical e se move para a esquerda quando $h$ se move para baixo no espaço de fases. Este comportamento é na verdade esperado por que quanto maior $h$ se torna, mais iteradas $n$ do mapeamento (3.1) são necessários até que a partícula atinja $h$. Um gráfico de $n_{p}$ vs. $h$ é mostrado na figura 3.4(c). Se a posição $h$ é reescalada com $h \rightarrow h / \epsilon$, as diferentes curvas de $n_{p}$ obtidas para diferentes $\epsilon$ são colapsadas em um único gráfico, como mostrado na figura $3.4(\mathrm{~d})$.

Vamos agora discutir sobre nossos resultados numéricos obtidos para o coeficiente de difusão. A partir de simulações numéricas, obtivemos diferentes valores para o coeficiente $A$ e a Eq. (3.6) foi avaliada. O coeficiente de difusão é mostrado na figura 3.3(a) para diferentes parâmetros de controle $\epsilon$ e $\gamma$. Diferentes valores do parâmetro de controle $\epsilon$ geraram diferentes valores para $D$ em concordância com $D / \epsilon^{2}=1 / 4$. Notamos que $D$ é quase constante para uma vasta faixa de $h$ até que repentinamente sofre um decréscimo. O decréscimo acontece porque a posição $h$ atingiu a ilha periódica mais baixa. A partir de análises da estabilidade de pontos fixos encontramos que a posição do último ponto fixo de período um, gerando a última ilha de período um é localizada em

$$
J_{\mathrm{LSI}} \cong\left[\frac{\gamma \pi \epsilon}{2}\right]^{\left[\frac{1}{1+\gamma}\right]}
$$

onde o índice LSI é usado para representar a ilha periódica mais baixa. Vemos que 
existem dois diferentes tipos de escalas para o comportamento de $D$. A primeira escala é observada para pequenos valores de $h$. Nesta região, existe provavelmente uma quebra da aproximação contínua usada na equação de difusão uma vez que $h$ se aproxima suficientemente da escala de variação de $\epsilon$. A figura 3.3(b) mostra o comportamento de $D$ para a reescala $D \rightarrow D / \epsilon^{2}$ e $h \rightarrow h / \epsilon$. Vemos claramente que todas as curvas de $D$ obtidas para diferentes parâmetros de controle, como mostrado na figura 3.3(a), se sobrepõe para pequenos valores de $h / \epsilon$ mas diferem para elevados valores de $h$. O resultado surpreendente é obtido quando o eixo horizontal é reescalado em relação à posição da ilha periódica mais baixa que é dado pela Eq. (3.7), i.e. $h \rightarrow h / J_{\mathrm{LSI}}$, como mostrado na figura 3.3(c). Em adição vemos que apesar da diferença de $D / \epsilon^{2}$ para diferentes parâmetros de controle $\epsilon$ considerando baixo $h$, o coeficiente de difusão sofre uma abrupta mudança quando a posição $h$ atinge as ilhas periódicas. Por causa das regiões periódicas, as partículas devem sofrer um aprisionamento temporário devido ao stickiness e isto influencia no transporte ao longo do espaço de fases. Neste ponto a difusão normal não é mais observada. Consequentemente o TLC não é aplicado neste domínio.

\subsection{Conclusões}

Obtivemos analiticamente coeficientes de difusão para diferentes sistemas e estudamos as propriedades de transporte de um conjunto de partículas clássicas para uma família de mapeamentos Hamiltonianos bidimensionais. As curvas de histograma de frequência do número de partículas que atingem uma altura $h$ em uma dada iterada $n$ foram obtidas numericamente e um ajuste exponencial foi feito. Mostramos que os coeficientes de difusão são invariantes de escala para diferentes posições do buraco. Os procedimentos adotados aqui também podem ser aplicados em outros sistemas com mares de caos e ilhas de periodicidade. No futuro seria interessante investigar se uma equação de difusão modificada (talvez fracionária) poderia ser aplicada a variação de $J$ na região de aprisionamento, e se o mesmo tipo de stickiness é presente na primeira curva invariante spanning. Este capítulo apresentou os resultados publicados na revista Physical Review $E$ (31) no ano de 2013. 
3. INVARIÂNCIA DE ESCALA DO COEFICIENTE DE DIFUSÃO EM UMA FAMÍLIA DE MAPEAMENTOS HAMILTONIANOS

BIDIMENSIONAIS 
$\Gamma_{\text {Capítulo }} 4$

\section{Poço de potencial dependente periodicamente do tempo}

\subsection{Resumo}

Estudaremos o modelo do poço de potencial, onde consideraremos partículas clássicas não interagentes que estão inseridas dentro de uma caixa com potenciais infinitos nas bordas. A caixa contém um poço de potencial dependente periodicamente do tempo em seu interior. O poço de potencial sofre a influência de diferentes perturbações temporais. A dinâmica de cada partícula é descrita por um mapeamento bidimensional não-linear e que preserva a área no espaço de fases nas variáveis energia e tempo. Isto leva a um espaço de fases contendo ilhas periódicas rodeadas por um extenso mar caótico e este último é limitado por um conjunto de curvas invariantes do tipo spanning. O histograma de frequência para o número de partículas que atingem uma certa energia $h$ em uma dada iterada $n$, ao qual observamos ser invariante de escala, cresce rapidamente até atingir um valor máximo e então decresce até atingir o valor zero para tempos suficientemente longos. Gráficos da probabilidade de sobrevivência da dinâmica em função do tempo são exponenciais para tempos curtos, atingindo um tempo de crossover e tendendo a um regime de baixo decaimento devido as regiões com grande stickiness no espaço de fases. Na última parte deste capítulo mostraremos numericamente que os expoentes obtidos (e consequentemente os observáveis médios no espaço de fases) não dependem das perturbações temporais que são propostas para o potencial móvel.

\subsection{Motivação}

Depois do resultado de Buttiker e Landauer (76) sobre tunelamento através de uma barreira de potencial dependente do tempo, o interesse na dinâmica de uma partícula em poços e barreiras dependentes do tempo tem crescido. Esta dinâmica pode ser caracteri- 


\section{POÇO DE POTENCIAL DEPENDENTE PERIODICAMENTE DO TEMPO}

zada tanto por trabalhos teóricos quanto experimentais, onde ambas as caracterizações clássicas e quânticas podem ser consideradas. Várias aplicações podem ser discutidas, incluindo a condutância balística em um canal periodicamente corrugado (77), transporte magnético através da heteroestrutura do GaAs/AlGaAs (39), influência do transporte na presença de micro-ondas (78), transporte anômalo em um guia de ondas corrugado (79), caracterização através do expoente de Lyapunov da dinâmica caótica e destruição de curvas invariantes spanning (80) e muitas outras $(81,82)$.

Estudaremos as propriedades dinâmicas e de transporte de partículas clássicas em tais sistemas. Possíveis alusões do potencial dependente do tempo considerado nesta seção podem ser feitas quando consideramos o potencial criado por átomos localizados em sequência ao longo de uma cadeia infinita e simétrica, o que pode representar, por exemplo, a heteroestrutura do GaAs/AlGaAs (39). As oscilações do poço podem representar o efeito de fônons ou banho térmico na cadeia infinita. Uma vez que o poço de potencial está ganhando energia do banho térmico, esta energia é transferida para as partículas. É importante salientar que o potencial considerado nesta tese pode também aprisionar temporariamente partículas, exemplos podem ser visto por exemplo em quantum dots (83).

Parte dos resultados que serão apresentados aqui foram publicados em 2011 na revista Physical Review E (32), em 2012 na Physica A (33) e em 2013 na Physics Letters $A(34)$.

\subsection{O modelo e o mapa}

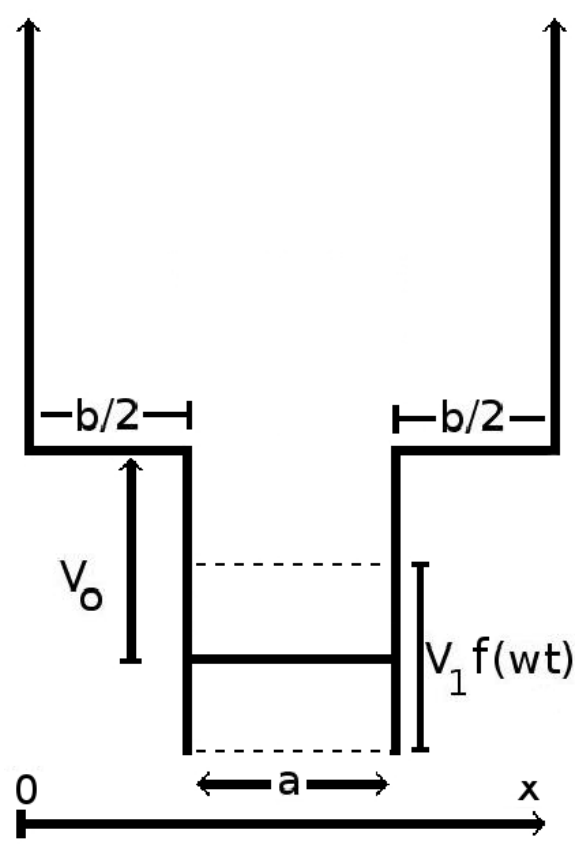

Figura 4.1: Esquema ilustrativo do potencial $V(x, t)$ dado pela Eq. (4.1). 
Consideraremos aqui uma partícula clássica de massa $m$ que se move ao longo do eixo $x$. Tal partícula sofre a influência de um poço de potencial com energia potencial dada por $V(x, t)$, onde $x$ é a posição da partícula e $t$ é o tempo. Um esquema típico pode ser visto na figura 4.1, onde a base do poço de potencial varia periodicamente no tempo de acordo com a função $f_{0}(t)=V_{1} f_{j}(\mathrm{w} t)$, onde $f_{j}$ pode ser qualquer função derivável em todos os pontos e que possa ser modulada em $2 \pi$, w é a frequência e $t$ é o tempo. Vemos que o poço se encontra no interior de uma caixa com potenciais infinitos em suas bordas.

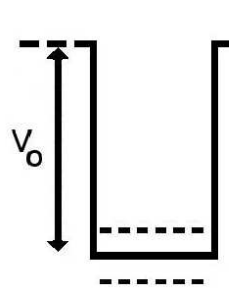

(a)

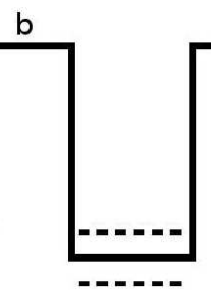

a

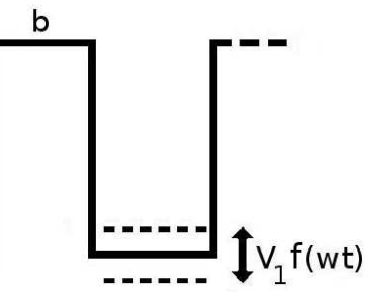

a

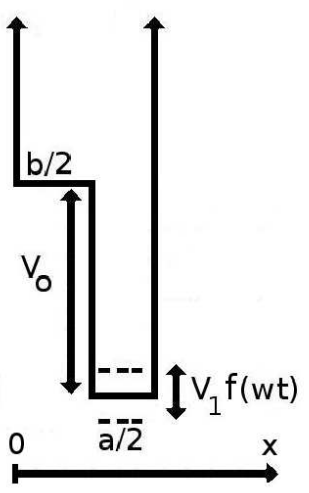

Figura 4.2: (a) Uma cadeia contendo infinitos poços de potencial simétricos, onde os fundos destes se movem periodicamente e sincronizados no tempo; (b) Um único poço de potencial; (c) Poço de potencial apresentado em (b) mas que foi cortado ao meio por causa de sua simetria.

Vale ressaltar que outros tipos de formas de potenciais também podem ser usados para obter a mesma descrição da dinâmica do sistema. Podemos, por exemplo, considerar uma cadeia de poços de potencial infinitos e simétricos dependentes do tempo, onde seus fundos se movem periódica e sincronizadamente no tempo, como é mostrado na figura 4.2(a). Considerando a geometria do problema, podemos também assumir um único poço de potencial que não contém uma caixa infinita de potencial, mas possui condições de contorno periódicas nas bordas, como mostrado na figura 4.2(b). Finalmente e mais genericamente, podemos considerar um poço de potencial em que o fundo se move periodicamente no tempo dentro de uma caixa de potencial infinito como podemos ver na figura $4.2(\mathrm{c})$. Entretanto, neste capítulo preferimos usar o esquema da 


\section{POÇO DE POTENCIAL DEPENDENTE PERIODICAMENTE DO TEMPO}

figura 4.1, onde o potencial $V(x, t)$ pode ser escrito como

$$
V(x, t)=\left\{\begin{array}{l}
\infty, \text { se } x \leq 0 \text { ou } x \geq(a+b) \\
V_{0}, \text { se } 0<x<\frac{b}{2} \text { ou }\left(a+\frac{b}{2}\right)<x<(a+b) \\
V_{1} f_{j}(\text { w } t), \text { se } \frac{b}{2} \leq x \leq\left(a+\frac{b}{2}\right)
\end{array},\right.
$$

onde $a, b, V_{0}, V_{1}$ e w são constantes.

Vamos supor que em um tempo $t=t_{n}$, a partícula inicia seu movimento com energia $E=E_{n}$ e está prestes a entrar no poço de potencial oscilante em $x=b / 2$. Ao entrar no poço a partícula sofre uma mudança abrupta em sua energia cinética, logo a energia cinética pode ser escrita como $K_{n}^{\prime}=E_{n}-V_{1} f_{j}\left(\mathrm{w} t_{n}\right)=\frac{1}{2} m v_{n}^{\prime 2}$ onde $\left|v_{n}^{\prime}\right|=\sqrt{2 K_{n}^{\prime} / m}$ é constante. Vale ressaltar que as velocidades são constantes, pois não temos forças de qualquer natureza atuando sobre a partícula. As exceções ocorrem apenas em $x=b / 2$ e $x=a+b / 2$ onde temos mudanças bruscas nas velocidades da partícula. Chegando no outro lado do poço (em $x=a+b / 2)$, a energia da partícula é $E_{n}^{\prime}=K_{n}^{\prime}+V_{1} f_{j}\left[\mathrm{w}\left(t_{n}+\Delta t_{n}^{\prime}\right)\right]$ onde $\Delta t_{n}^{\prime}=a /\left|v_{n}^{\prime}\right|$. Se a partícula não tem energia suficiente para escapar do poço ela é refletida de volta, ou seja, $E_{n}^{\prime} \leq V_{0}$. Além de retornar, ela sofrerá reflexões sucessivas até que tenha energia suficiente para deixar o poço. Podemos então reescrever $E_{n}^{\prime}$ como $E_{n}^{\prime}=K_{n}^{\prime}+V_{1} f_{j}\left[\mathrm{w}\left(t_{n}+i \Delta t_{n}^{\prime}\right)\right]$ onde $i$ é o menor inteiro que satisfaz a condição $E_{n}^{\prime}>V_{0}$, condição esta que assegura que a partícula escapou.

Uma vez que a partícula escapou do poço oscilante, ela viajará em direção a uma das bordas da caixa com potenciais infinitos, sofrerá uma colisão elástica deste e irá em direção ao poço oscilante. A nova energia imediatamente antes de entrar no poço é dada por $E_{n+1}=E_{n}+V_{1}\left\{f_{j}\left[\mathrm{w}\left(t_{n}+i \Delta t_{n}^{\prime}\right)\right]-f_{j}\left(\mathrm{w} t_{n}\right)\right\}$. O tempo é escrito como $t_{n+1}=t_{n}+i \Delta t_{n}^{\prime}+\Delta t_{n}^{\prime \prime}$ onde $\Delta t_{n}^{\prime \prime}=b /\left|v_{n}^{\prime \prime}\right| \operatorname{com}\left|v_{n}^{\prime \prime}\right|=\sqrt{2 K_{n}^{\prime \prime} / m}$ e $K_{n}^{\prime \prime}=E_{n+1}-V_{0}$.

Podemos ver que existem muitos parâmetros de controle e que alguns não são relevantes para descrever a dinâmica do sistema. No total temos 5 , estes são $a, b, V_{0}, V_{1}$ e w. Definiremos variáveis adimensionais afim de diminuir o número de parâmetros de controle: $\delta=V_{1} / V_{0}, r=b / a, e_{n}=E_{n} / V_{0}, N_{c}=\mathrm{w} /(2 \pi)\left(a / \sqrt{2 V_{0} / m}\right)$ e a medida do tempo em termos do número de oscilações do poço móvel, $\phi=$ wt. O parâmetro $N_{c}$ corresponde ao número de oscilações que o poço completa em um tempo $t=a / \sqrt{2 V_{0} / m}$, tempo que uma partícula, com energia cinética igual a $V_{0}$, levaria para percorrer a distância $a$ se o poço fosse estático.

Tendo em mente os parâmetros de controle propostos, o mapa pode ser reescrito como (34)

$$
T:\left\{\begin{array}{l}
e_{n+1}=e_{n}+\delta\left[f_{j}\left(\phi_{n}+i \Delta \phi_{a}\right)-f_{j}\left(\phi_{n}\right)\right] \\
\phi_{n+1}=\phi_{n}+i \Delta \phi_{a}+\Delta \phi_{b} \bmod (2 \pi)
\end{array},\right.
$$

onde as variáveis auxiliares são dadas por

$$
\begin{gathered}
\Delta \phi_{a}=\frac{2 \pi N_{c}}{\sqrt{e_{n}-\delta f_{j}\left(\phi_{n}\right)}}, \\
\Delta \phi_{b}=\frac{2 \pi N_{c} r}{\sqrt{e_{n+1}-1}} .
\end{gathered}
$$


Consideramos $i$ o menor inteiro que torna a expressão $e_{n}+\delta\left[f_{j}\left(\phi_{n}+i \Delta \phi_{a}\right)-f_{j}\left(\phi_{n}\right)\right]>1$ verdadeira.

Nos primeiros resultados que mostraremos, sempre consideraremos que a função $f$ é escrita da seguinte forma: $f_{0}(\phi)=\cos (\phi)$. Somente na última seção (4.7) alteraremos as funções $f_{j}$ e algumas propriedades dinâmicas serão discutidas.

\subsection{Espaço de fase e pontos fixos}

O espaço de fase do modelo é misto contendo ilhas periódicas, um extenso mar caótico e curvas invariantes do tipo spanning que separam diferentes regiões caóticas e limitam o crescimento da energia $e$, como podemos ver na figura 4.3.

Dadas as expressões do mapeamento, podemos obter os correspondentes pontos fixos. A descrição completa dos pontos fixos e de suas estabilidades foi feita no artigo publicado na revista Physica A (33), aqui reproduziremos parte dos resultados encontrados. Os pontos fixos de período um, sem múltiplas reflexões (ou seja, $i=1$ no mapa (4.2)), são obtidos fazendo $e_{n+1}=e_{n}=e^{*}$ e $\phi_{n+1}=\phi_{n}+2 m \pi=\phi^{*}$, onde $m$ é um inteiro positivo. Fazendo $e_{n+1}=e^{*}$ na primeira expressão do mapa (4.2), obtemos

$$
\delta\left[\cos \left(\phi^{*}+\Delta \phi_{a}\right)-\cos \left(\phi^{*}\right)\right]=0,
$$

e isso só é possível se $\delta=0$ (situação que não nos interessa, pois teríamos de ignorar esse parâmetro do mapa) ou $\cos \left(\phi^{*}+\Delta \phi_{a}\right)=\cos \left(\phi^{*}\right)$. Essa última condição nos remete a duas expressões para $\Delta \phi_{a}$ :

$$
\Delta \phi_{a}=k 2 \pi,
$$

$\mathrm{Ou}$

$$
\Delta \phi_{a}=k 2 \pi-2 \phi^{*},
$$

onde $k$ é um número inteiro maior que zero. Nomearemos de tratamento (i) quando utilizarmos $\Delta \phi_{a}$ da Eq. (4.6) e tratamento (ii) quando utilizarmos $\Delta \phi_{a}$ da Eq. (4.7). Após alguns cálculos, como resultado para o tratamento (i), encontramos que a energia no ponto fixo é

$$
e^{*}=1+\left(\frac{N_{c} r}{m-k}\right)^{2},
$$

onde $m$ e $k$ são constantes. A fase obtida pode ser igual à

$$
\phi^{*}=\arccos \left\{\frac{1}{\delta}\left[e-\left(\frac{N_{c}}{k}\right)^{2}\right]\right\},
$$

$\mathrm{Ou}$

$$
\phi^{*}=2 \pi-\arccos \left\{\frac{1}{\delta}\left[e-\left(\frac{N_{c}}{k}\right)^{2}\right]\right\} .
$$




\section{POÇO DE POTENCIAL DEPENDENTE PERIODICAMENTE DO} TEMPO

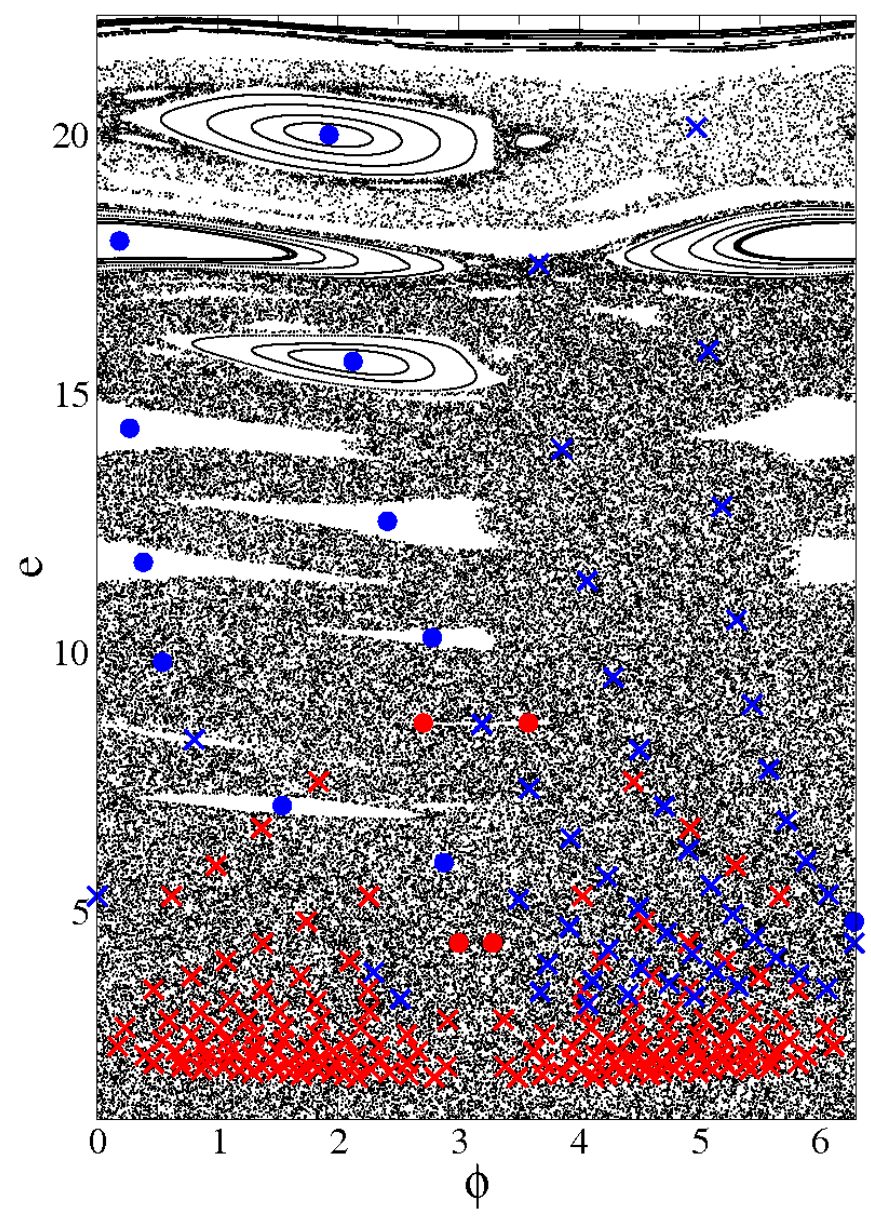

Figura 4.3: Espaço de fase para o mapeamento (4.2). Os círculos correspondem aos pontos fixos elípticos ao passo que aqueles em formato de cruz são pontos fixos hiperbólicos. As cores vermelhas são usadas no primeiro tratamento (i) e as azuis no segundo tratamento (ii). Os parâmetros de controle usados são $r=1, N_{c}=33,18$ e $\delta=0,5$.

Para o tratamento (ii) não é possível obtermos uma expressão analítica como solução para o par $\left(e^{*}, \phi^{*}\right)$, sendo necessário uso de solução numérica. É possível mostrar que a energia do ponto fixo é dada por:

$$
e^{*}=\delta \cos \left(\phi^{*}\right)+\left(\frac{\pi N_{c}}{k \pi-\phi^{*}}\right)^{2}
$$

Ao fazer $\phi_{n+1}=\phi^{*}+m 2 \pi$ na segunda expressão do mapa (4.2) e substituindo a expressão de $\Delta \phi_{a}$ da Eq. (4.7) e $\Delta \phi_{b}$ da Eq. (4.4) encontramos que

$$
1-e^{*}+\left[\frac{\pi N_{c} r}{\pi(m-k)+\phi^{*}}\right]^{2}=0
$$


e finalmente substituindo a expressão de $e^{*}$ da Eq. (4.11), obtemos

$$
1+\left[\frac{\pi N_{c} r}{\pi(m-k)+\phi^{*}}\right]^{2}-\delta \cos \left(\phi^{*}\right)+\left(\frac{\pi N_{c}}{k \pi-\phi^{*}}\right)^{2}=0,
$$

onde esta expressão nos fornece a fase $\left(\phi^{*}\right)$ dos pontos fixos de período 1 para o tratamento (ii). A energia é encontrada substituindo $\phi^{*}$ na Eq. (4.11).

Os pontos fixos podem ser caracterizados se olharmos os autovalores $\lambda$ da matriz Jacobiana $J$. Estes podem ser encontrados fazendo $\operatorname{Det}(J-\lambda I)=0$, onde Det é a determinante e $I$ é uma matriz identidade. Desta forma, obtemos $\lambda^{2}-\lambda(\operatorname{Tr}(J))+$ $\operatorname{Det}(J)=0$, onde a solução desta equação nos fornece $\lambda_{ \pm}=\frac{\operatorname{Tr}(J) \pm \sqrt{(\operatorname{Tr} J)^{2}-4 \operatorname{Det}(J)}}{2}$. A Jacobiana $J$ é escrita como

$$
J=\left(\begin{array}{cc}
\frac{\partial e_{n+1}}{\partial e_{n}} & \frac{\partial e_{n+1}}{\partial \phi_{n}} \\
\frac{\partial \phi_{n+1}}{\partial e_{n}} & \frac{\partial \phi_{n+1}}{\partial \phi_{n}}
\end{array}\right)
$$

e $\operatorname{Tr}(J)$ é dito o traço da Jacobiana, ou seja, a soma dos elementos da diagonal principal da matriz $J, \operatorname{Tr}(J)=\frac{\partial e_{n+1}}{\partial e_{n}}+\frac{\partial \phi_{n+1}}{\partial \phi_{n}}$.

Como o determinante da matriz Jacobiana é igual à 1 , o sistema preserva a área no espaço de fases. Os pontos fixos para o modelo são classificados da seguinte forma (5):

- Se $|\operatorname{Tr} J|<2$, o ponto fixo $\left(X^{*}, Y^{*}\right)$ é elíptico, ou seja, $\lambda_{ \pm}$assumem valores imaginários.

- Se $|\operatorname{Tr} J|=2$, o ponto fixo $\left(X^{*}, Y^{*}\right)$ é parabólico, neste caso $\lambda_{+}=\lambda_{-}=\operatorname{Tr} J / 2$.

- Se $|\operatorname{Tr} J|>2$, o ponto fixo $\left(X^{*}, Y^{*}\right)$ é hiperbólico, ou seja, $\lambda_{ \pm}$são números reais.

Na figura 4.3 podemos ver os pontos fixos para os dois tratamentos. Os parâmetros de controle usados são $r=1, N_{c}=33,18$ e $\delta=0,5$. Os círculos correspondem aos pontos fixos elípticos e os em formato de cruz são pontos fixos hiperbólicos. Pontos fixos elípticos são estáveis, ou seja, dada uma condição inicial exatamente no ponto fixo, a órbita nunca sairá deste ponto, ao contrário dos hiperbólicos que tem trajetórias que divergem do ponto fixo (ponto fixo instável). Ressaltamos que as cores vermelhas são usadas no primeiro tratamento (i) e as azuis no segundo tratamento (ii).

\subsection{Resultados - Propriedades de transporte através do mar caótico}

Nesta seção mostraremos os resultados sobre transporte no poço de potencial simples. Os resultados apresentados nesta seção foram publicados recentemente na revista Physical Review $E$ (32). Fixaremos os parâmetros de controle $r=1$, que recupera o caso em que o poço é simétrico, e $\delta=0,5$. Os resultados numéricos serão obtidos basicamente como função de $N_{c}$, que de forma simplificada, representa a frequência de 


\section{POÇO DE POTENCIAL DEPENDENTE PERIODICAMENTE DO TEMPO}
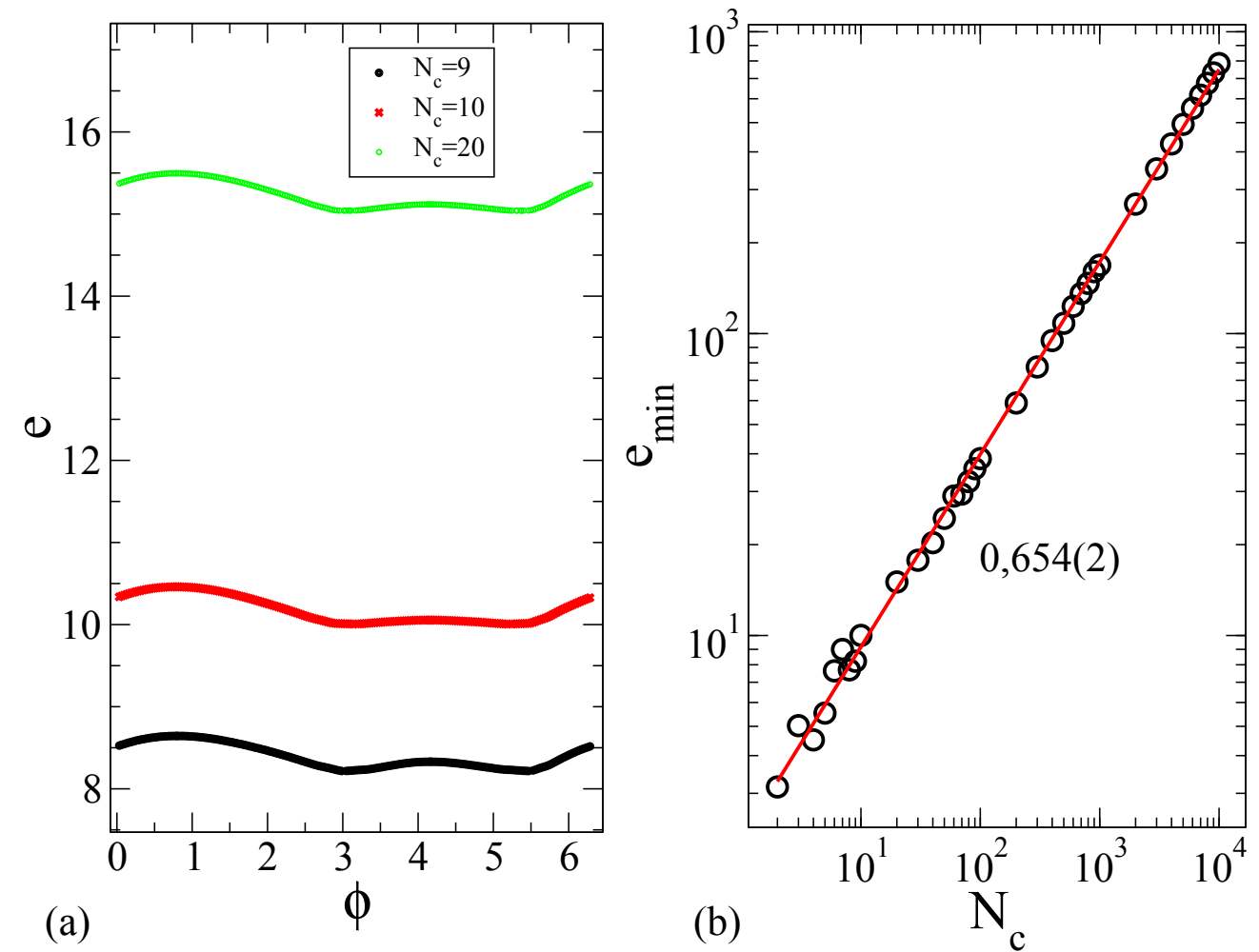

Figura 4.4: (a) Posição da primeira curva invariante do tipo spanning utilizando os parâmetros de controle $N_{c}=9, N_{c}=10$ e $N_{c}=20$. (b) Gráfico de $e_{\min } \times N_{c}$. Os parâmetros de controle utilizados foram $r=1$ e $\delta=0,5$.

oscilação do potencial móvel. Se aumentarmos o valor de $N_{c}$, as variáveis auxiliares $\Delta \phi_{a}$ e $\Delta \phi_{b}$ (definidas pelas equações (4.3) e (4.4)), que dependem linearmente de $N_{c}$, também terão seus valores aumentados. Tal fato diminui a correlação entre $\phi_{n+1}$ e $\phi_{n}$, fazendo com que a posição da primeira curva invariante aumente de valor no espaço de fases. Para confirmar tal fato, temos a posição aparente da primeira curva invariante utilizando $N_{c}=9, N_{c}=10$ e $N_{c}=20$, como mostrado na figura 4.4(a). Para construir tal figura, dividimos o eixo $\phi$ em 1000 intervalos igualmente espaçados e anotamos o valor da máxima energia obtida para cada intervalo após $10^{11}$ iteradas de uma única órbita (com $\phi_{0}=0$ e $e_{0}=1,0001$ ), seguramente localizada no mar de caos.

Um gráfico da energia mínima ao longo da curva invariante spanning como função de $N_{c}$ é mostrado na figura 4.4(b), onde após uma ajuste em lei de potência temos como resultado $\alpha_{1}=0,654(2) \cong 2 / 3$. É importante salientar que a posição da curva invariante de mais baixa energia é descrita pela mesma lei de potência que descreve o comportamento do desvio da energia média como função deste mesmo parâmetro (para maiores detalhes, aconselhamos a leitura do artigo publicado na revista Physica A, ver $(33))$.

Investigaremos agora o transporte de partículas no espaço de fases. Consideraremos 


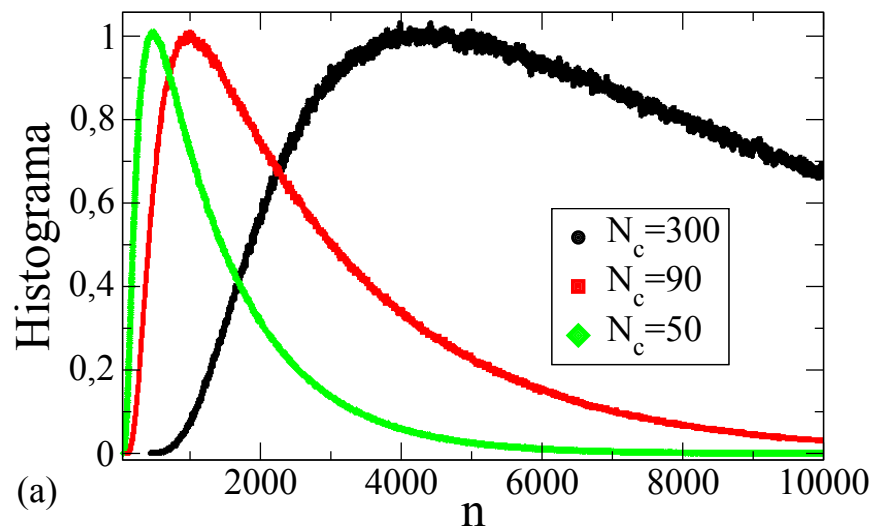

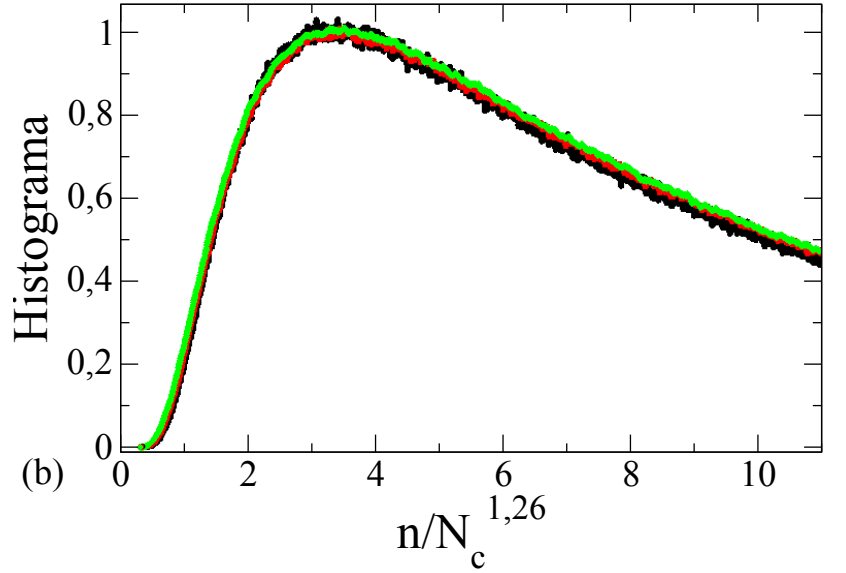

Figura 4.5: (a) Gráfico do histograma do número de partículas que atingiram a altura $h$ em um certo número de iteradas $n$ para diferentes valores de $N_{c}$. (b) Colapso de diferentes histogramas em uma única curva, confirmando uma invariância de escala. Os outros parâmetros usados nas simulações foram: $r=$ 1 e $\delta=0,5$.

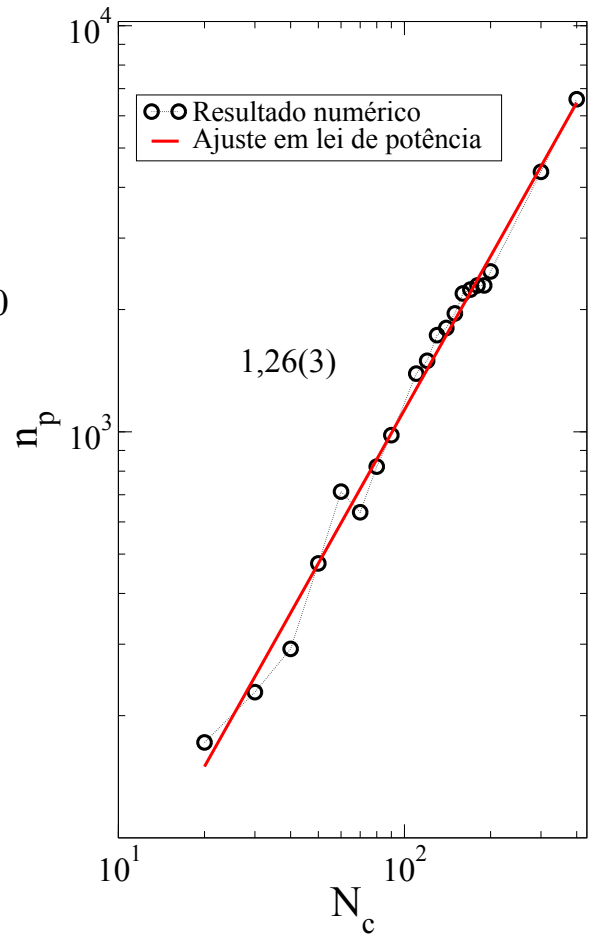

Figura 4.6: Gráfico de $n_{p} \times N_{c}$. Um ajuste em lei de potência fornece $\gamma=1,26(3)$.

que se uma partícula atingir ou ultrapassar uma certa energia (ou altura) $h$, devemos anotar o número de iteradas dadas e uma nova condição será iniciada. Consideraremos em nossas simulações $h=0,7 e_{\text {min }}$, embora outro valor possa também ser utilizado. Começaremos as simulações com um conjunto de $10^{7}$ partículas com baixa energia inicial $\left(e_{0}=1,01\right)$ e com diferentes fases iniciais $\phi_{0} \in[0,2 \pi)$, que serão evoluídas no tempo. Um gráfico do histograma de frequência para o número de partículas com $e \geq h$ em uma dada iteração $n$ é mostrado na figura 4.5(a). É importante observar que todos os gráficos de histograma de frequência foram normalizados à 1 para melhor visualização dos dados. Podemos ver que para tempos curtos o número de partículas que atingem $h$ é pequeno. Esse número cresce na medida em que $n$ aumenta, atingindo um máximo em $n=n_{p}$ e então se aproxima de zero assimptoticamente. Vemos também que quanto 


\section{POÇO DE POTENCIAL DEPENDENTE PERIODICAMENTE DO TEMPO}
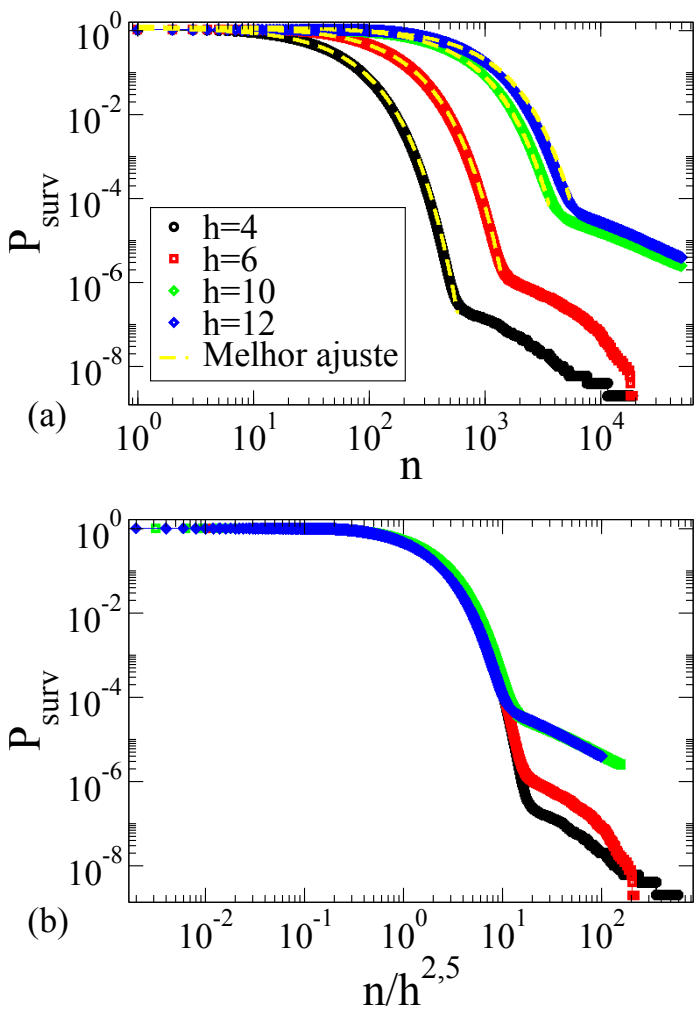

Figura 4.7: (a) Gráfico de $P$ vs $n$ para diferentes posições de $h(h=4,6,10 \mathrm{e}$ 12). Os parâmetros de controle usados foram $N_{c}=33,18, r=1$ e $\delta=0,5$. As linhas pontilhadas são obtidas através de um ajuste exponencial. (b) Colapso, para pequenos valores de $n$, de todas as curvas mostradas em (a) depois de uma reescala

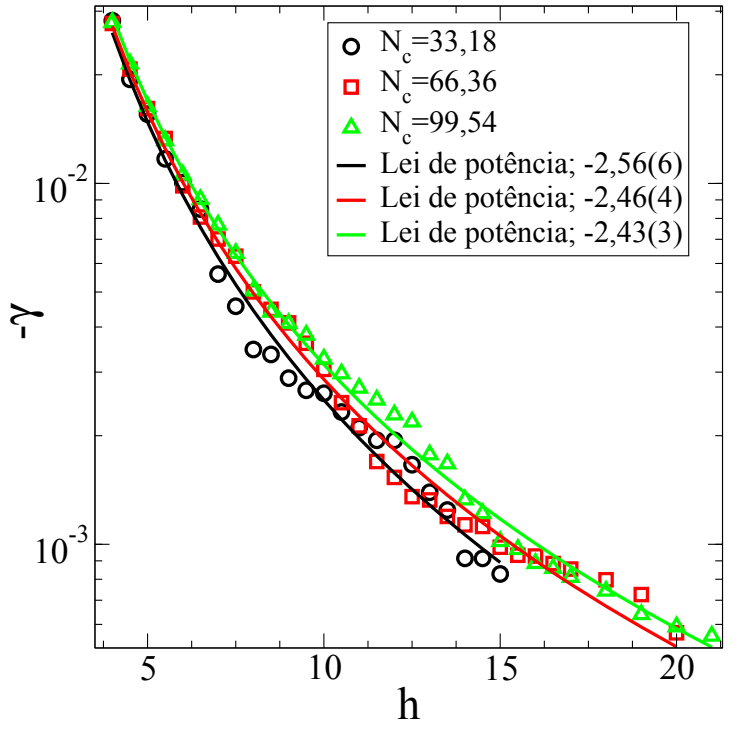

Figura 4.8: Expoente do decaimento exponencial $\gamma$ como função do $h$ para três diferentes valores de $N_{c}: N_{c}=33,18, N_{c}=$ 66,36 e $N_{c}=99,54$. Os outros parâmetros de controle utilizados foram $r=1$ e $\delta=$ 0,5 . As linhas retas são o melhor ajuste para cada $N_{c}$ escolhido. $n \rightarrow n / h^{2,5}$.

maior é o parâmetro $N_{c}$, maior será $n_{p}$, nos levando a supor que $n_{p} \propto N_{c}^{\gamma}$. Um gráfico de $n_{p}$ em função de $N_{c}$ nos fornece $\gamma=1,26(3)$, conforme mostrado na figura 4.6. Este comportamento nos faz supor que $n_{p}$ é invariante de escala. Isto significa que uma reescala apropriada no eixo $n$, isto é, $n \rightarrow n / N_{c}^{\gamma}$, irá colapsar todas as curvas em uma única curva universal. Isto é comprovado na figura 4.5(b).

Discutiremos o comportamento da probabilidade de sobrevivência, que é definida como (13)

$$
P(t)=\int_{t}^{\infty} p_{e}\left(t^{\prime}\right) d t^{\prime},
$$

onde $p_{e}(t)$ é densidade de probabilidade de escape. Para o caso discreto, consideraremos 
$P$ como

$$
P=\frac{1}{N} \sum_{i=1}^{N} n_{\text {surv }}^{(i)},
$$

onde a soma é tomada ao longo de um conjunto de $N$ condições iniciais diferentes e $n_{\text {surv }}$ é o número de órbitas que não tem energia suficiente para atingir $h$. É conhecido na literatura (13) que quando a Eq. (4.15) é considerada ao longo de uma órbita completamente caótica, o comportamento de $P$ é um decaimento exponencial (13). Porém quando órbitas periódicas estão coexistindo no espaço de fases, a probabilidade de sobrevivência tem um decaimento mais lento, que pode culminar em uma lei de potência quando o fenômeno de stickiness é muito intenso (84).

Obtemos o comportamento de $P$ em função de $n$ para diferentes posições do buraco, como mostrado nas figuras 4.7(a) e 4.7(b). Vemos que o comportamento inicial, é claramente um decaimento exponencial até atingir um crossover e mudar para um decaimento mais lento, indicando a existência de regiões de aprisionamento no espaço de fases. O expoente de decaimento em função da posição do buraco pode ser visto na figura 4.8. A figura 4.7(b) mostra um colapso das diferentes curvas de $P$ mostrados na figura 4.7(a) para pequenos valores de $n$, de todas as curvas mostradas na figura 4.7(a) após uma reescala $n \rightarrow n / h^{2,5}$, confirmando uma invariância de escala da probabilidade de sobrevivência para pequenos valores de $n$. Colapso similar foi observado para diferentes combinações de parâmetros e diferentes energias iniciais.

\subsection{Conclusões parciais}

Estudamos o problema de uma partícula clássica confinada dentro de uma caixa com potenciais infinitos nas bordas e contendo um poço de potencial oscilante. O transporte de energia foi visto ser invariante de escala da frequência de oscilações do poço para baixas energias iniciais. O histograma de frequência do número de partículas que atingiram uma certa altura $h$ em uma dada iterada, para tempos curtos, foi medido e caracterizado como invariante sob uma escala apropriada. A probabilidade de sobrevivência em função de $n$ era inicialmente exponencial, atinge um crossover, seguido de um lento decaimento para tempos longos. Ambos os decaimentos exponenciais e mais lentos são evidências de um aprisionamento de partículas próximo das ilhas de periodicidade no espaço de fases. Os resultados apresentados até esta parte do capítulo foram publicados em 2011 na revista Physical Review E (32) e em 2012 na Physica A (33).

\subsection{Poço de potencial com diferentes perturbações tem- porais}

Como dito nas seções anteriores, sempre consideramos que a função que descrevia o movimento do potencial móvel era dado por $f_{0}(\phi)=\cos (\phi)$. Agora consideraremos que 
(a)
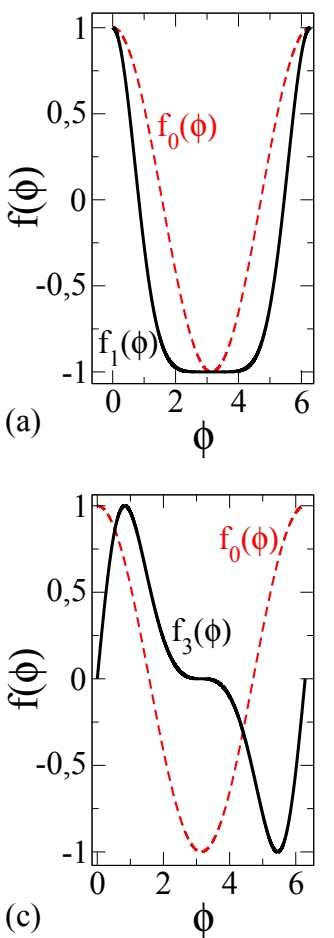
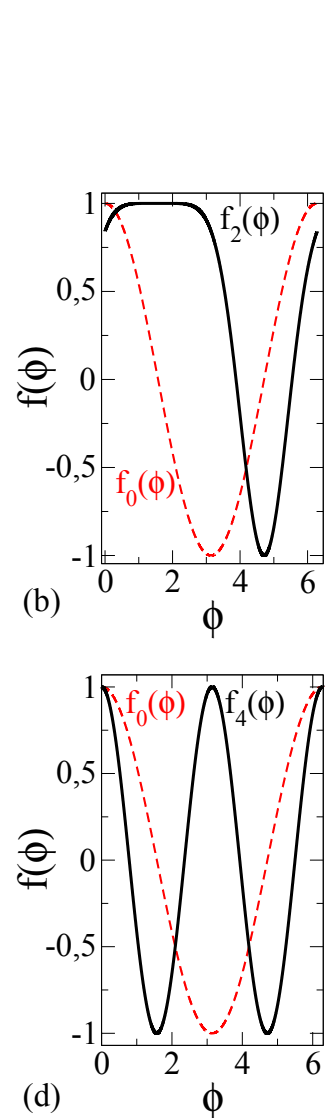

\section{0}

30

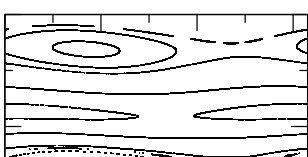

(1) $20 \Rightarrow$

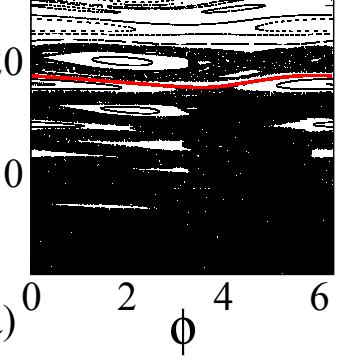

(a)

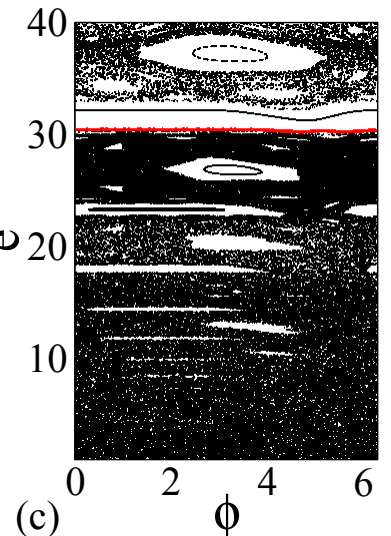

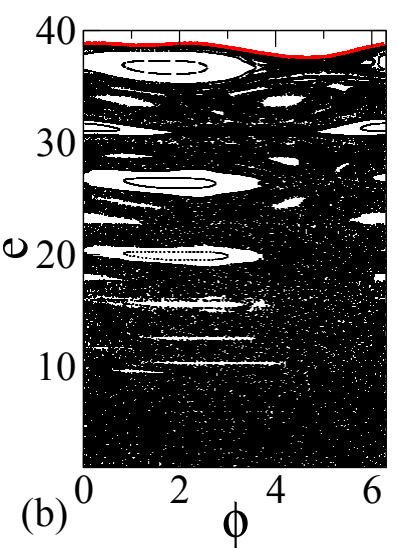

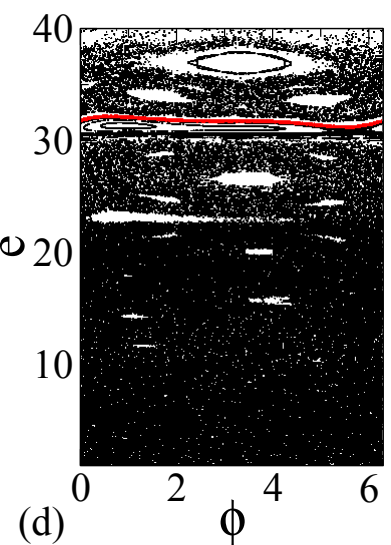

Figura 4.9: Gráfico das funções: (a) $f_{1}(\phi) ;\left(\right.$ b) $f_{2}(\phi) ;\left(\right.$ c) $f_{3}(\phi) ;($ d) $f_{4}(\phi)$ usando $q=2$. As linhas pontilhadas vermelhas correspondem a função $f_{0}(\phi)$ que foi usada como comparação.

Figura 4.10: Espaço de fases para o mapeamento (4.2) usando como parâmetros de controle $N_{c}=33,18, r=1$ e $\delta=0,5$ para: (a) $f_{0}(\phi),(\mathrm{b}) f_{1}(\phi),(\mathrm{c}) f_{2}(\phi)$ e $(\mathrm{d}) f_{3}(\phi)$. As curvas vermelhas representam a posição da primeira curva invariante spanning.

a função $f_{j}$ pode assumir diferentes expressões, como mostrado na figura 4.9(a,b,c,d). As funções $f_{1}(\phi), f_{2}(\phi), f_{3}(\phi)$ e $f_{4}(\phi)$ são dadas por

$$
f_{1}(\phi)=\cos [\phi+\sin (\phi)], \quad f_{2}(\phi)=\sin [\phi+\cos (\phi)],
$$

e

$$
f_{3}(\phi)=\sin [\phi+\sin (\phi)], \quad f_{4}(\phi)=\cos (q \phi) .
$$

onde $q$ é um número inteiro positivo. Para $q=1$ a função $f_{0}(\phi)=\cos [\phi]$ é recuperada. Resultados obtidos para a dinâmica de partículas considerando a função $f_{4}(\phi)$ serão mostrados mais adiante. É importante salientar que quaisquer funções poderiam ter sido propostas nas $f_{j}$, porém decidimos utilizar estas funções pois elas geram espaços de fases que têm diferenças significativas. Outro motivo de termos escolhidos, por exemplo, a função $f_{1}$ é que ao observarmos a figura 4.9(a) vemos que esta função fica mais tempo 
próxima do valor -1 ao variamos $\phi$ do que quando comparado com a função $f_{0}$. O oposto ocorre com a função $f_{2}$, neste caso a função se aproxima de +1 para uma grande faixa de $\phi$. A função $f_{3}$ tem um pequeno platô constante próximo de 0 . Finalmente, para a função $f_{4}$ podemos por exemplo considerar $q$ tendendo ao infinito, desta forma perderíamos a correlação entre a energia nos instantes $n$ e $n+1$, fazendo com que a função trabalhe de forma quase aleatória.

O espaço de fases para o mapeamento (4.2) é mostrado na figura 4.10(a,b,c,d) para diferentes tipos de perturbações e considerando fixos os parâmetros de controle $N_{c}=33,18, r=1$ e $\delta=0,5$. Consideramos $r=a / b=1$ pois recupera o caso onde o poço potencial é simétrico, em outras palavras, $a=b$. Considerando $\delta=V_{1} / V_{0}=0,5$ implica que a amplitude do movimento do poço móvel é metade do potencial $V_{0}$. Já o valor de $N_{c}=33,18$ foi considerado pois de acordo com a Ref. (54), este caso representa uma frequência moderada de oscilações. Vemos que o espaço de fases para todas as funções é misto. Quando a função $f_{j}$ (com $\left.j=0,1,2,3\right)$ é mudada, deformações em algumas ilhas periódicas podem ser vistas e a posição dos pontos fixos também muda. A posição da primeira curva invariante spanning claramente depende da função que é considerada.

Vamos agora discutir o comportamento do expoente de Lyapunov. A figura 4.11(a) mostra o gráfico de $\lambda$ vs $n$ para $f_{0}$ e considerando quatro diferentes condições iniciais, onde $\phi_{0}$ é escolhido aleatoriamente no intervalo $\phi_{0} \in(0,2 \pi]$ para os parâmetros $N_{c}=10$ (que foi escolhido por ser da mesma ordem de grandeza de $N_{c}=33.18$ ), $r=1$ e $\delta=0,5$. A energia inicial usada foi $e_{0}=1,001$, o que garante que para todo o valor de $\phi_{0}$ teremos uma órbita caótica. Vemos que após uma flutuação inicial, o expoente de Lyapunov positivo converge para um valor constante para valores suficientemente grandes de $n$. Os platôs de saturação dependem dos valores dos parâmetros de controle. O expoente de Lyapunov médio é dado por

$$
\bar{\lambda}=\frac{\sum_{i=1}^{N} \lambda_{i}}{N}
$$

onde $\lambda_{i}$ é obtido como o comportamento assintótico para órbitas caóticas considerando diferentes condições iniciais. Em nossas simulações consideramos $N=5$ e iteramos o mapeamento $10^{8}$ vezes para cada órbita. Valores elevados de $N$ produzem uma pequena redução das barras de erro. Na figura 4.11(b,c,d) temos respectivamente os gráficos de $\bar{\lambda}$ como função de $N_{c}, r$ e $\delta$ para $f_{0}, f_{1}, f_{2}$ e $f_{3}$. Da figura 4.11(b), vemos que o expoente de Lyapunov positivo varia de $\bar{\lambda} \approx 0,5$ para $N_{c}=1$ até $\bar{\lambda} \approx 2$ para $N_{c}=10^{3}$. Este também tem uma tendência monotônica de crescimento como função de $N_{c}$ para todos os $f_{j}(j=0, \ldots, 3)$. Note entretanto que aumentar o valor de $N_{c}$ corresponde a aumentar o número de oscilações do poço. Isso implica que em um mesmo intervalo de tempo, o fundo do poço oscila um número maior de vezes com o aumento de $N_{c}$. Este fato leva a um aumento do expoente de Lyapunov. A figura 4.11(c) mostra o gráfico de $\bar{\lambda}$ vs $r$ para valores fixos de $\delta=0,5$ e $N_{c}=33,18$ considerando diferentes funções $f_{j}$. Aumentar o valor de $r$ para valores fixos de $N_{c}$ e $\delta$ corresponde a aumentar a distância $b$, consequentemente a distância que as partículas percorrem na região do potencial que não depende do tempo é maior. Estes longos voos até a próxima entrada no 


\section{POÇO DE POTENCIAL DEPENDENTE PERIODICAMENTE DO TEMPO}

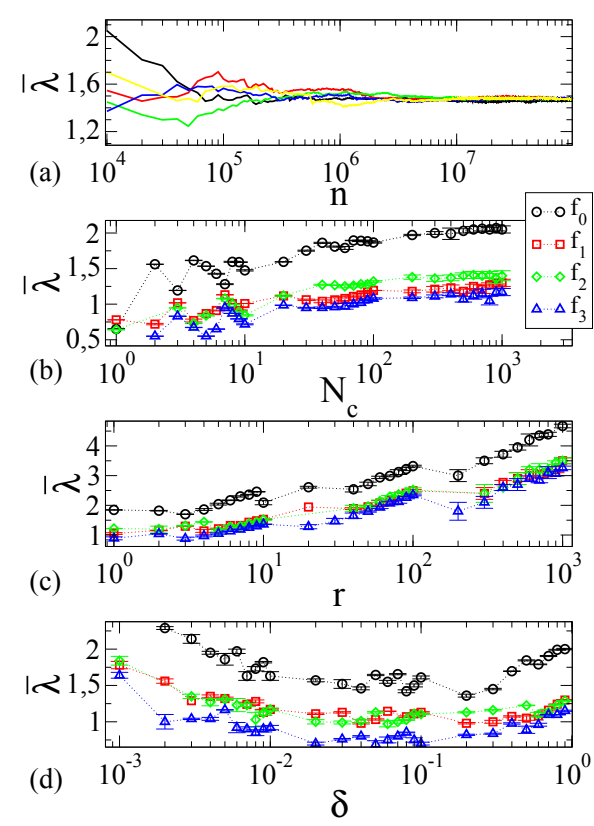

Figura 4.11: (a) Gráfico do expoente de Lyapunov como função de $n$ para cinco diferentes condições iniciais e considerando a função $f_{0}$. Os parâmetros usados foram $N_{c}=10, r=1 \mathrm{e}$ $\delta=0,5$. Para as funções $f_{0}, f_{1}$, $f_{2}$ e $f_{3}$ temos: (b) $\bar{\lambda}$ vs $N_{c}$ para valores fixos de $r=1$ e $\delta=0,5$; (c) $\bar{\lambda}$ vs $r$ para $N_{c}=33,18 \mathrm{e}$ $\delta=0,5 ;$ (d) $\bar{\lambda}$ vs $\delta$ considerando $N_{c}=33,18$ e $r=1$.
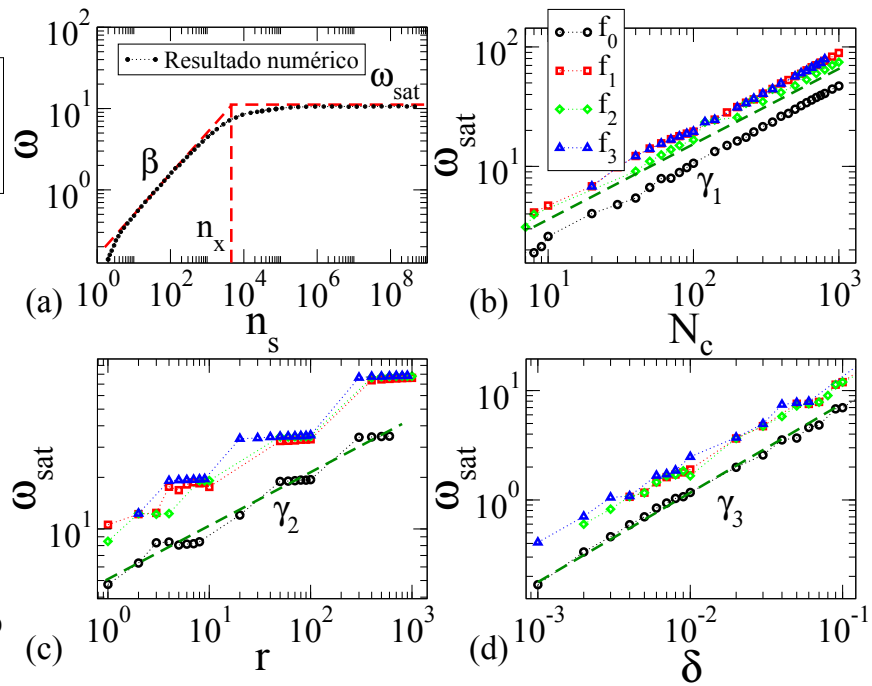

Figura 4.12: Gráfico de: (a) $\omega$ vs $n$ usando $f_{0}$ e considerando $N_{c}=100, r=1$ e $\delta=0,5$. Para as funções $f_{0}, f_{1}, f_{2}$ e $f_{3}$ temos: (b) $\omega_{\text {sat }}$ vs $N_{c}$ para valores fixos de $r=1$ e $\delta=0,5$; (c) $\omega_{\text {sat }}$ vs $r$ para $N_{c}=33,18$ e $\delta=0,5$ (d) $\omega_{\text {sat }}$ vs $\delta$ considerando $N_{c}=300$ e $r=1$. Valores numéricos para os expoentes são mostrados na Tabela 4.1.

potencial móvel acarretam em um aumento do número de oscilações e consequentemente da 'aleatoriedade' do sistema. Os saltos abruptos no comportamento do Lyapunov médio são explicados como destruições de curvas invariantes spanning, levando a uma junção de diferentes regiões caóticas. Maiores detalhes serão mostrados na seção. 4.7.3. Finalmente, um gráfico $\bar{\lambda}$ vs $\delta$ é mostrado na figura 4.11(d). Os parâmetros de controle usados foram $r=1$ (poço simétrico) e $N_{c}=33,18$ (gera uma frequência moderada de oscilações). Vemos que pequenos valores de $\delta$, ao qual correspondem a pequenas flutuações do potencial oscilante, produzem altos valores do expoente de Lyapunov. Um valor mínimo de $\bar{\lambda}$ vs $\delta$ para todos $f_{j}$ foi observado para $\delta \approx 0,1$. 


\subsubsection{Desvio da energia média ao longo do mar caótico}

Agora discutimos o comportamento do desvio da energia média para o regime caótico de baixas energias. Para tal usamos a energia média, definida como

$$
\bar{e}\left(n, \delta, N_{c}, r\right)=\frac{1}{n} \sum_{i=1}^{n} e_{i},
$$

e então o desvio da energia média é escrito como

$$
\omega\left(n, \delta, N_{c}, r\right)=\frac{1}{M} \sum_{j=1}^{M} \sqrt{\overline{e_{j}^{2}}\left(n, \delta, N_{c}, r\right)-{\overline{e_{j}}}^{2}\left(n, \delta, N_{c}, r\right)},
$$

onde $M$ denota um conjunto de condições iniciais diferentes.

Tabela 4.1: Expoentes obtidos analisando o desvio da energia média.

\begin{tabular}{lllll}
\hline \hline & $f_{0}$ & $f_{1}$ & $f_{2}$ & $f_{3}$ \\
\hline$\alpha_{1}$ & $0,659(4)$ & $0,651(3)$ & $0,66(2)$ & $0,649(6)$ \\
$\alpha_{2}$ & $0,32(1)$ & $0,30(1)$ & $0,331(9)$ & $0,30(1)$ \\
$\alpha_{3}$ & $0,78(1)$ & $0,76(1)$ & $0,725(8)$ & $0,74(2)$ \\
$z_{1}$ & $1,31(2)$ & $1,30(3)$ & $1,26(2)$ & $1,28(2)$ \\
$z_{2}$ & $0,64(2)$ & $0,61(2)$ & $0,66(2)$ & $0,62(3)$ \\
$z_{3}$ & $-0,65(3)$ & $-0,55(4)$ & $-0,59(2)$ & $-0,59(6)$ \\
\hline \hline
\end{tabular}

A figura 4.12(a) mostra o gráfico de $\omega$ vs $n$ para $N_{c}=100, r=1, \delta_{r}=0,5$ e considerando a função $f_{0}$. Vemos que $\omega$ cresce como lei de potência para pequenos valores de $n$ e com expoente $\beta \approx 0,5$ e, após um número de crossover $n_{x}, \omega$ tende a um regime de saturação atingindo $\omega_{\text {sat }}$ para valores de $n$ suficientemente grandes. Variações dos parâmetros $N_{c}, r$ e $\delta$ produzem gráficos similares mas com diferentes valores de $n_{x}$ e saturações. Desta forma, propomos que

- (i) Para $n \ll n_{x}$, o comportamento de $\omega$ pode ser descrito como

$$
\omega\left(n \delta^{2}, N_{c}, r, \delta\right) \propto\left[n \delta^{2}\right]^{\beta},
$$

onde $\beta$ é um expoente de aceleração. Após diversas simulações, concluímos que $\beta \cong 1 / 2$;

- (ii) Para $n \gg n_{x}, \omega_{\text {sat }}$ é dado por

$$
\omega_{\text {sat }}\left(n \delta^{2}, N_{c}, r, \delta\right) \propto N_{c}^{\alpha_{1}} r^{\alpha_{2}} \delta^{\alpha_{3}},
$$

onde $\alpha_{1}, \alpha_{2}$ e $\alpha_{3}$ são expoentes; 


\section{POÇO DE POTENCIAL DEPENDENTE PERIODICAMENTE DO TEMPO}
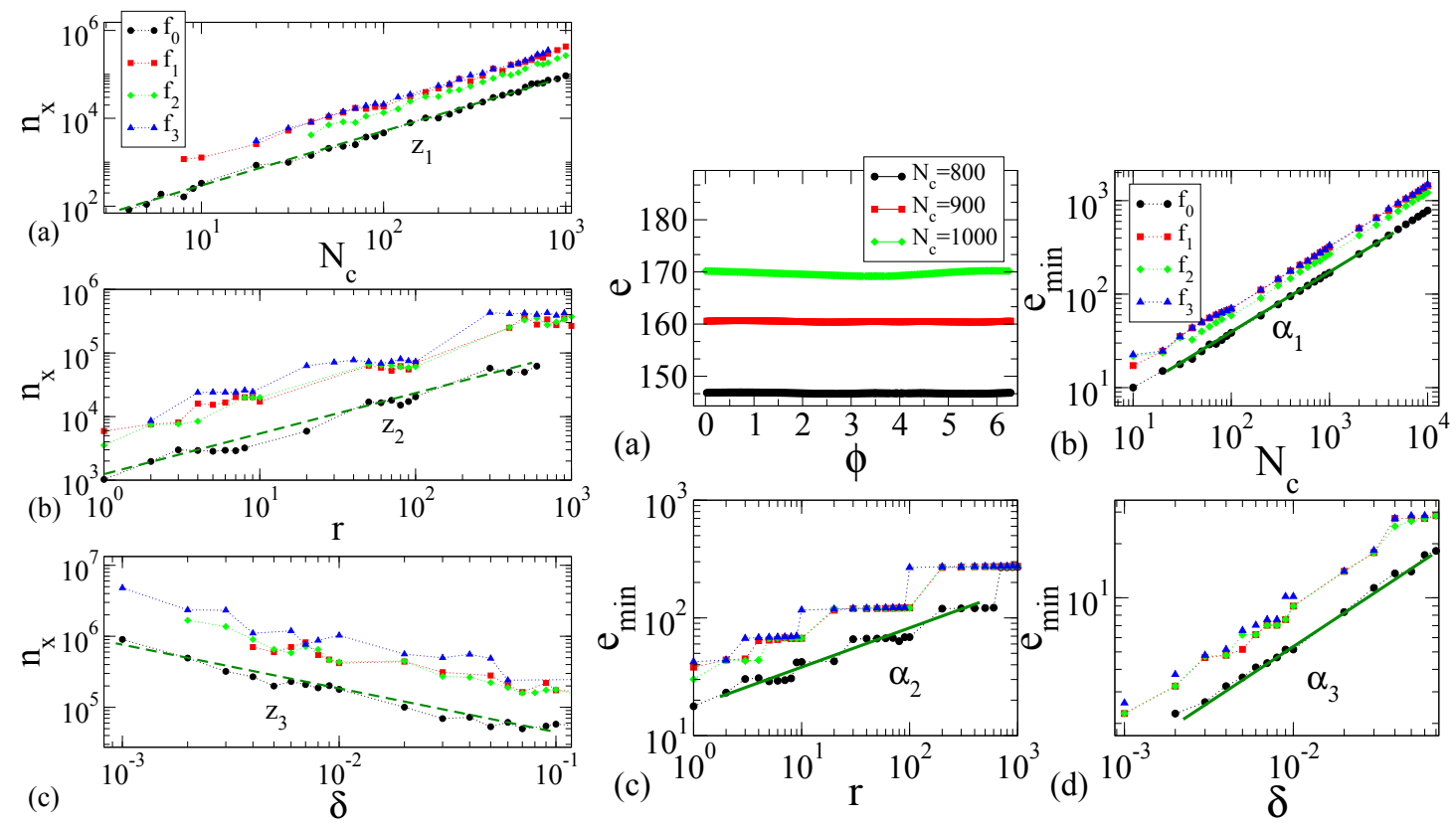

Figura 4.13: Gráfico do crossover $n_{x}$ como função dos parâmetros de controle, considerando diferentes funções $f_{0}$, $f_{1}, f_{2}$ e $f_{3}$ para: (a) $n_{x}$ vs $N_{c}$ para valores fixos de $r=1$ e $\delta=0,5 ;$ (b) $n_{x}$ vs $r$ para $N_{c}=33,18$ e $\delta=0,5$ (c) $n_{x}$ vs $\delta$

Figura 4.14: (a) Gráfico da posição da curva invariante spanning usando a função $f_{0}$ e considerando $r=1$ e $\delta=0,5$ para $N_{c}=800, N_{c}=900 \mathrm{e}$ $N_{c}=1000$. (b) Gráfico de $e_{\min }$ vs $N_{c}$ com inclinação $\alpha_{1}$ para diferentes funções $f_{i}(i=0,1,2,3)$; (c) $e_{\min }$ vs $r$ com inclinação $\alpha_{2}$; (d) $e_{\min }$ vs $\delta$ com inclinação $\alpha_{3}$. para $N_{c}=300$ e $r=1$.

- (iii) O número característico de crossover $n_{x}$ é escrito como

$$
n_{x}\left(n \delta^{2}, N_{c}, r, \delta\right) \propto N_{c}^{z_{1}} r^{z_{2}} \delta^{z_{3}},
$$

onde $z_{1}, z_{2}$ e $z_{3}$ também são expoentes.

Os expoentes podem ser encontrados analisando o comportamento de $\omega_{\text {sat }}$ e $n_{x}$ em função dos parâmetros de controle. Na figura 4.12(b) temos um gráfico de $\omega_{\text {sat }}$ vs $N_{c}$. Aplicando um ajuste em lei de potência obtemos os valores dos expoentes $\alpha_{i}$ com $i=1,2,3$, conforme mostrado na Tabela 4.1 , para $f_{0}, f_{1}, f_{2}$ e $f_{3}$. Usando procedimento similar mas agora variando os parâmetros $r$ e $\delta$ (ver figura 4.12(c) e figura 4.12(d)) encontramos $\alpha_{2} \approx 1 / 3$ e $\alpha_{3}$ como mostrado na Tabela 4.1.

O comportamento do crossover $n_{x}$ também pode ser caracterizado como função dos parâmetros de controle. Da figura 4.13(a,b,c) e aplicando ajustes em lei de potência, obtivemos para cada função $\left(f_{0}, f_{1}, f_{2}\right.$ e $\left.f_{3}\right)$ os expoentes $z_{1}, z_{2}$ e finalmente $z_{3}$, como mostrado na Tabela 4.1. 
Tabela 4.2: Expoentes obtidos pela mínima energia ao longo da curva invariante spanning.

\begin{tabular}{lllll}
\hline \hline & $f_{0}$ & $f_{1}$ & $f_{2}$ & $f_{3}$ \\
\hline$\alpha_{1}$ & $0,657(1)$ & $0,657(1)$ & $0,645(4)$ & $0,661(2)$ \\
$\alpha_{2}$ & $0,37(2)$ & $0,31(3)$ & $0,33(1)$ & $0,29(2)$ \\
$\alpha_{3}$ & $0,64(1)$ & $0,66(2)$ & $0,660(9)$ & $0,64(2)$ \\
\hline \hline
\end{tabular}

\subsubsection{Localização da curva invariante spanning}

Vamos agora estudar detalhes da curva invariante spanning. Esta funciona como uma barreira física que previne o crescimento ilimitado de energia. A posição da curva depende dos valores dos parâmetros de controle adotados. A figura 4.14(a) mostra a posição da primeira curva invariante spanning considerando valores fixos de $r=1 \mathrm{e}$ $\delta=0,5$ e para $N_{c}=800, N_{c}=900$ e $N_{c}=1000$ (valores que correspondem a uma frequência de oscilações elevada, conforme a Ref. (54)), onde consideramos a função $f_{0}$. Um gráfico da energia mínima ao longo da curva invariante spanning $\left(e_{\min }\right)$ como função dos parâmetros fornece uma prévia de como o mar caótico se comporta. A figura 4.14(b) mostra $e_{\min }$ vs $N_{c}$ e a correspondente inclinação é $\alpha_{1}$. Usando um procedimento similar, gráficos de $e_{\min }$ vs $r$ e $e_{\min }$ vs $\delta$ são mostrados na figura 4.14(c,d) com suas respectivas inclinações. $\alpha_{1}, \alpha_{2}$ e $\alpha_{3}$ obtidos para diferentes funções $f_{0}, f_{1} f_{2}$ e $f_{3}$ são mostrados na Tabela 4.2. Notamos que o valor numérico de $\alpha_{1} \cong 2 / 3$ é bem descrito pelas predições teóricas da Ref. (67).

\subsubsection{Comportamento da energia como função do parâmetro $r$}

Discutiremos agora algumas propriedades do espaço de fases como função do parâmetro $r$. Como mostrado na figura 4.14(c), $e_{\min }$ se mantém constante para certos intervalos de $r$ e então muda de valor abruptamente. Isto é facilmente observado para a faixa de $r=100$ e $r=200$ e também para o intervalo $r=600$ e $r=700$. Nas figuras 4.15(a-f) temos grades de $10^{6}$ condições iniciais igualmente espaçadas com $\phi_{0} \in[0,2 \pi)$ e $e_{0} \in[60,74]$. As cores nas figuras. 4.15(a-c) representam o logaritmo do tempo de escape (LTE) para cada condição inicial escolhida. Definimos o tempo de escape como o número de iterações do mapeamento até que a energia seja $e>75$ ou $e<$ 59. O número máximo de iterações usados foi $10^{6}$. As cores brancas correspondem a regiões onde as partículas foram aprisionadas para a condição inicial escolhida e não escapam até $10^{6}$ iteradas. De fato, para algumas regiões as partículas permanecem aprisionadas para sempre como se estivessem em ilhas periódicas. Se $e>75$ ou $e<59$, consideramos que a partícula escapou e uma nova órbita é iniciada. Como mostrado na figura 4.15(a) (com $r=80$ ) existe uma vasta região (cor branca) onde o tempo de escape é maior que $10^{6}$. A cor vermelha representa as regiões onde o stickiness é observado, com isto elevando o tempo de escape quando comparamos com regiões sem stickiness. Tais regiões predominantemente aparecem próximas de ilhas periódicas. As cores na figura 4.15(d-f) representam o número de diferentes tempos de retorno (NDTR). Para calcular este observável, consideramos um retângulo de recorrência, onde toda vez que 


\section{POÇO DE POTENCIAL DEPENDENTE PERIODICAMENTE DO TEMPO}

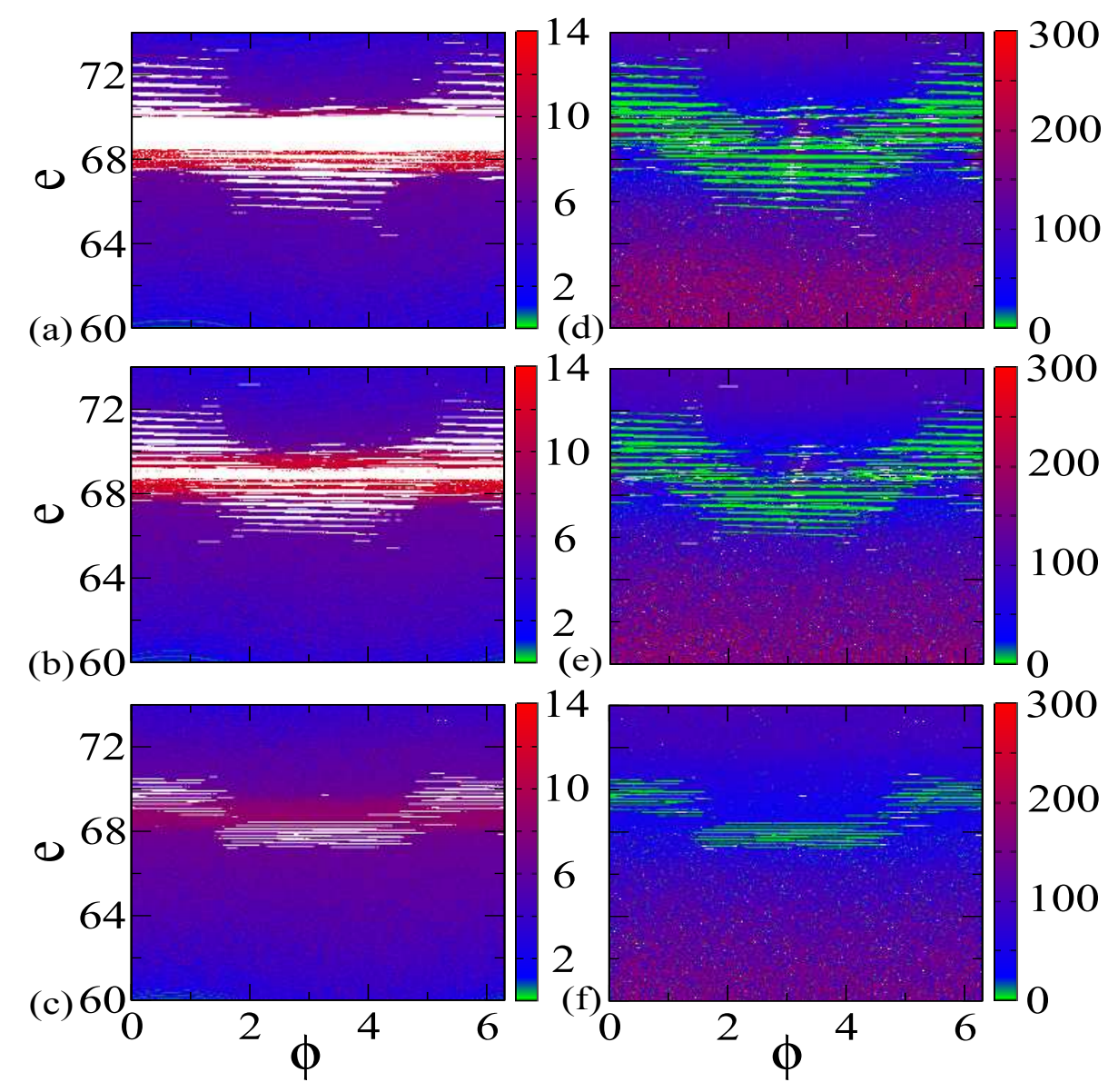

Figura 4.15: Grade de condições iniciais $\left(\phi_{0}, e_{0}\right)$ mostrando o logaritmo do tempo de escape (LTE) para: (a) $r=80$; (b) $r=100$; (c) $r=200$. Número de diferentes tempos de retorno (NDTR) para: (d) $r=80$; (e) $r=100$; (f) $r=200$. As janelas de escape são situadas em $e_{1}<59$ e $e_{1}>75$. A função $f_{0}$ foi usada para obter os resultados.

a partícula entra nesta região um contador é acumulado e este conta o número de diferentes tempos de retorno. Para o mesmo parâmetro $r=80$, como mostrado na figura 4.15(d) vemos que dentro das ilhas periódicas, o NDTR têm valores baixos (cor verde). De acordo com as figuras $4.15(\mathrm{~d}, \mathrm{e}, \mathrm{f})$ uma região caótica é caracterizada por NDTR $\cong 300$, isto significa que temos vários tempos de retornos diferentes, ao passo que em regiões com stickiness temos valores de NDTR da ordem de 50, pois as órbitas tem momentaneamente um movimento quasi-periódico.

A figura 4.15(b) mostra os resultados para LTE usando $r=100$. Vemos que a região branca diminui quando comparada com a figura 4.15(a). Por outro lado, os resultados para NDTR são aproximadamente os mesmos daqueles obtidos na figura 4.15(e). Para $r=200$ e como mostrado na figura 4.15(c) para a análise da LTE, concluímos que é possível atravessar a região com stickiness (as regiões brancas desapareceram), com isso 


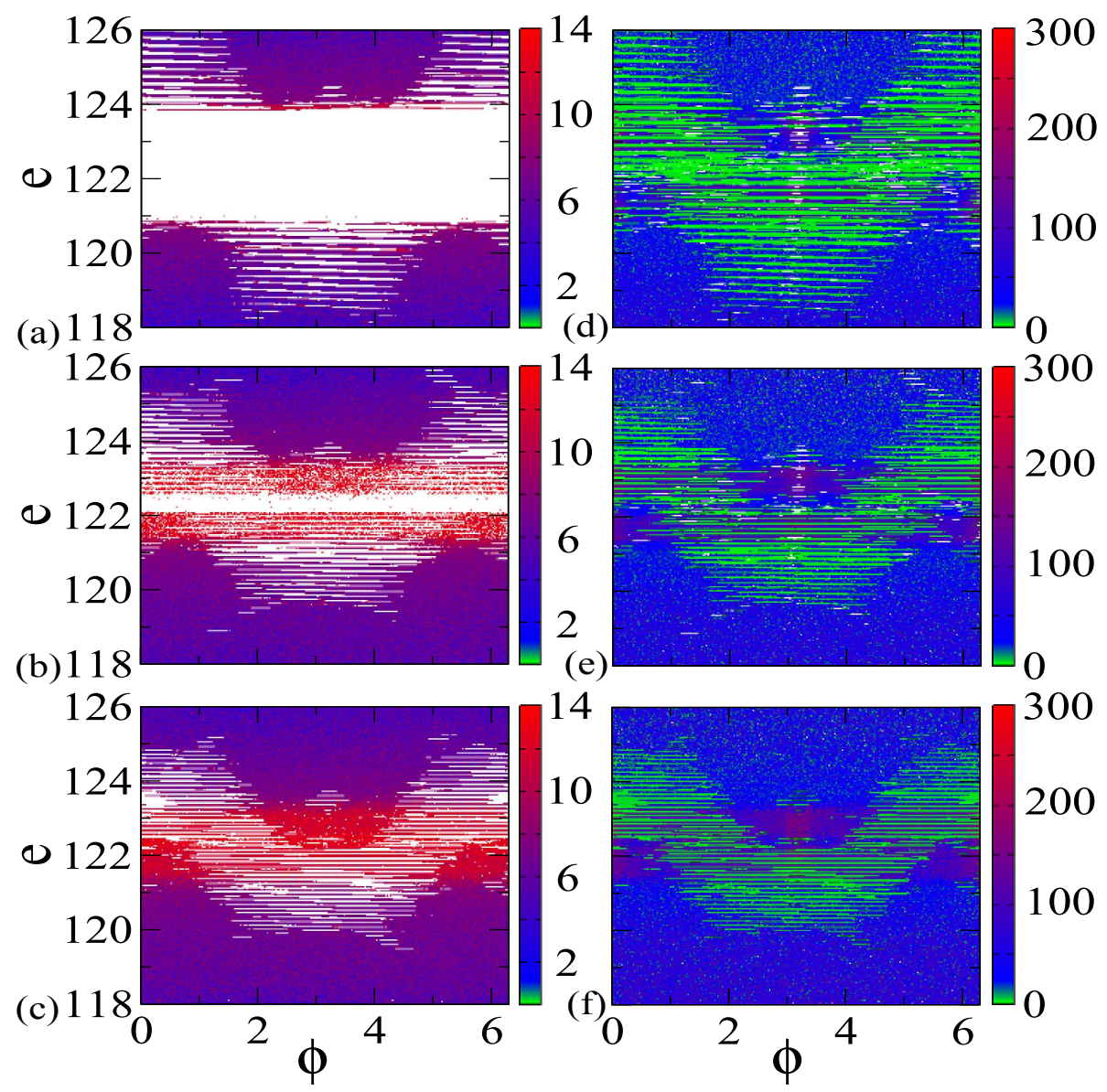

Figura 4.16: Grade com diferentes condições iniciais $\left(\phi_{0}, e_{0}\right)$ mostrando como cores o logaritmo do tempo de escape (LTE) para: (a) $r=400$; (b) $r=600$; (c) $r=700$. Número de diferentes tempos de retorno (NDTR) para: (d) $r=400 ;$ (e) $r=600$; (f) $r=700$. As janelas de escape são situadas em $e_{1}<117$ e $e_{1}>127$. A função $F_{0}$ foi usada para obter os resultados.

a energia das partículas deve crescer. Olhando a figura 4.15(f), o NDTR confirma que a região sticky se torna menor e então as ilhas começam a desaparecer. Consequentemente as órbitas atingem uma maior energia máxima $e \cong 120$, como mostrado na figura 4.16(a) para $r=400$. Novamente é possível observar as regiões brancas no LTE e consequentemente stickiness destacados na NDTR (ver figura 4.16(d)). Para valores mais altos de $r$, digamos $r=600$, de novo as regiões brancas se tornam menores no LTE (ver figura 4.16(b)), mas para $r=700$ (figura 4.16(c)) tais regiões desaparecem e a energia cresce. Tal fenômeno acontece para valores maiores de $r$ e podemos concluir que os degraus de energia, como mostrados na figura 4.14(c) para $e_{\min }$ aparecem por causa de extensas regiões de stickiness. 


\section{POÇO DE POTENCIAL DEPENDENTE PERIODICAMENTE DO TEMPO}

\subsubsection{Resultados para a função $f_{4}(\phi)$}
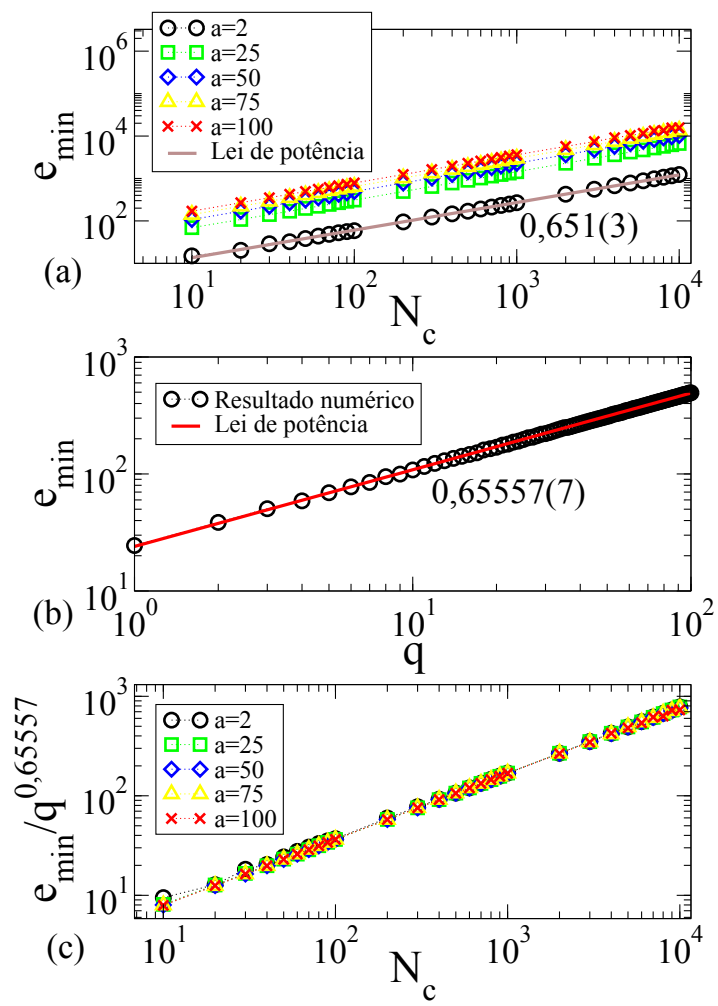

Figura 4.17: Gráfico de $e_{\min }$ vs $N_{c}$ para $q=2, q=25, q=50$ e $q=100$. Um ajuste em lei de potência nos fornece a inclinação $2 / 3$; (b) Gráfico de $e_{\min }$ vs $q$ com inclinação 0,65557(7); (c) Colapso das curvas mostradas no item (a) em um único e universal gráfico depois da transformação $e_{\min } \rightarrow e_{\min } / a^{0,65557}$.

De acordo com a definição da função $f_{4}$ (ver equação Eq. 4.17) para $q=1 \mathrm{a}$ função $f_{0}(\phi)$ é recuperada. Aumentando $q$, o número de picos e vales também aumenta para $\phi \in[0,2 \pi]$. A figura $4.9(\mathrm{~d})$ confirma esta suposição para $q=2$. Claramente um aumento em $q$ produz um aumento do número de oscilações no fundo do poço do sistema com isto elevando a posição da curva invariante spanning. A figura 4.17(a) mostra um gráfico de $e_{\min }$ vs $N_{c}$ para quatro diferentes valores de $q$. Como podemos ver, depois de um ajuste em lei de potência a inclinação obtida para cada curva é aproximadamente igual a 2/3. A figura 4.17(b) mostra um gráfico de $e_{\min }$ vs $q$, e a inclinação obtida foi $\alpha=0,65557(7)$. Usando tais resultados e reescalando apropriadamente os eixos da figura 4.17(a) ( $\left.e_{\min } \rightarrow e_{\min } / a^{0,65557}\right)$ mostramos que a posição do mínimo da curva invariante spanning é invariante de escala em relação a $q$. 


\subsection{Conclusões}

Estudamos a dinâmica de um conjunto de partículas clássicas confinadas em uma caixa com potenciais infinitos na borda e que contêm poços de potencial com diferentes funções perturbadoras. Um dos resultados mais interessantes foi mostrar que os expoentes de escala são independentes da função perturbadora que é proposta como perturbação para o fundo do poço de potencial. É importante dizer que os expoentes descrevem o comportamento característico de um conjunto de partículas no espaço de fases e é controlado pela posição da primeira curva invariante spanning. Os expoentes $\alpha_{i}(i=1,2,3)$ são obtidos analisando a saturação do desvio da energia média em função dos parâmetros de controle. O expoente $\beta$ foi encontrado e seu valor é próximo de $1 / 2$ para todas as funções $f_{j}(j=0, \ldots, 4)$. Os expoentes $z_{i}(i=1,2,3)$ foram obtidos analisando o crossover $n_{x}$ como função dos parâmetros de controle. Discutimos o comportamento da energia da partícula como função do parâmetro $r$, o qual tem um comportamento diferente, mostrando faixas com valores de energia constante quando variamos o valor do parâmetro. Foi observado que uma região com bastante stickiness evita a difusão da partícula ao longo do mar caótico, com isto aprisionando as partículas por tempos suficientemente longos. Em relação a função $f_{4}$, mostramos que a posição do mínimo da curva invariante spanning é invariante de escala com relação ao parâmetro $q$. Os resultados mostrados nesta última parte da seção foram publicados na revista Physics Letters A em 2013 (34). 
4. POÇO DE POTENCIAL DEPENDENTE PERIODICAMENTE DO TEMPO 


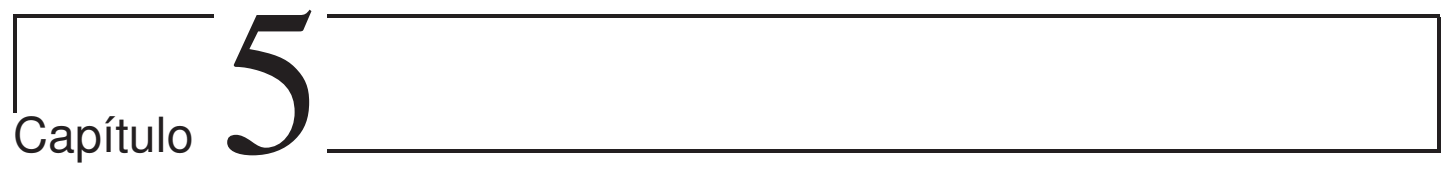

\section{Bilhar ovóide girante}

\subsection{Resumo}

Algumas propriedades dinâmicas para um bilhar ovóide com fronteiras girantes são estudadas neste capítulo. Duas situações são possíveis: curvatura (i) positiva ou (ii) negativa. Para o caso (i), vemos que o fenômeno de aceleração de Fermi (crescimento ilimitado de energia) não é observado, o que aparentemente é uma contradição da conjectura proposta por Loskutov-Ryabov-Akinshin (86). Para o caso (ii) mostramos que a velocidade média para um conjunto de partículas não interagentes cresce com uma lei de potência com expoente 0,1763 . Mostramos também que há uma analogia entre o bilhar girante e bilhares magnéticos ao analisarmos as curvas de Larmor formadas. Os resultados mostrados neste capítulo foram publicados na Communications in Nonlinear Science 83 Numerical Simulation (35).

\subsection{Motivação}

Uma extensão natural de sistemas unidimensionais são os bilhares bidimensionais. Em tais sistemas temos partículas não interagentes confinadas dentro de uma região fechada e experimentando colisões com a fronteira (85). Basicamente estes podem ser classificados em três diferentes classes, (i) integrável, (ii) ergódico e (iii) mista. Uma das principais questões sobre tais sistemas é a presença ou não de aceleração de Fermi. Neste sentido a conjectura proposta por Loskutov, Ryabov e Akinshin (LRA) (86) basicamente diz que se um sistema com fronteiras estáticas tem componentes caóticas no espaço de fases, então uma perturbação temporal na fronteira seria condição suficiente para produzir o crescimento ilimitado de energia. Tal conjectura foi confirmada em diversos bilhares, por exemplo, oval (87), estádio (88), gaz de Lorentz (89) e muitos outros. Recentemente foi mostrado que mesmo para bilhares elípticos (90, 91), cuja integrabilidade vem da conservação do momento angular em relação aos dois focos, depois de introduzir dependência temporal na fronteira, o crescimento ilimitado de energia é observado $(90,92)$. Simulações mostraram que condições iniciais escolhidas ao 


\section{BILHAR OVÓIDE GIRANTE}

longo da curva separatriz do bilhar com fronteiras estáticas levavam a partícula à exibir aceleração de Fermi (fenômeno que leva a um crescimento ilimitado de energia). Então a conjectura LRA pode ser estendida para a existência de uma órbita heteroclínica no espaço de fases ao invés da existência de um conjunto com dinâmica caótica. O mecanismo que gera aceleração de Fermi neste caso são os sucessivos cruzamentos de uma partícula pela vizinhança da curva separatriz no caso estático, ao qual sob perturbação temporal da fronteira se torna uma camada estocástica. Tais cruzamentos mudam a dinâmica da partícula de rotação para libração (ou ao contrário) e causa uma flutuação da energia cinética da partícula. Tais flutuações aumentam com o tempo e levam a uma difusão anômala e consequentemente aceleração de Fermi (90, 92). Neste capítulo estudaremos o sistema com uma perturbação do tipo giro, ou seja, a fronteira do bilhar gira com velocidade angular constante.

\subsection{O modelo e o mapa}

Discutiremos os passos para construir as equações do mapeamento que descrevem a dinâmica do sistema. O modelo consiste de uma partícula clássica confinada dentro de um bilhar ovóide dependente periodicamente do tempo e que experimenta colisões elásticas com a fronteira. A dinâmica do sistema é descrita em termos de um mapeamento não-linear e quadridimensional $T\left(\theta_{n}, \alpha_{n},\left|\vec{V}_{n}\right|, t_{n}\right)=\left(\theta_{n+1}, \alpha_{n+1},\left|\vec{V}_{n+1}\right|\right.$ ,$\left.t_{n+1}\right)$ onde as variáveis denotam: $(\theta)$ a posição angular da partícula; $(\alpha)$ o ângulo que a trajetória da partícula forma com a linha tangente na posição da colisão; $\left(\left|\vec{V}_{n+1}\right|\right)$ a velocidade absoluta da partícula e; $(t)$ o instante de colisão com a fronteira. Na figura 5.1 temos um esboço da fronteira oval e dos ângulos envolvidos na descrição do mapa. A forma da fronteira em coordenadas polares pode ser escrita como

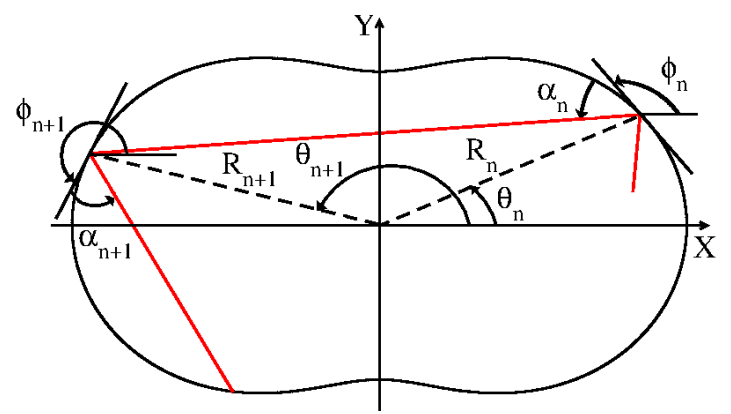

Figura 5.1: Esquema ilustrativo da fronteira e dos ângulos envolvidos na descrição do mapa. Nesta figura temos que $p=2$ e $\epsilon>\epsilon_{c}=0,2$.

$$
R_{b}(\theta, p, \epsilon, t)=1+\epsilon \cos \left[p \theta^{\prime}(t)\right],
$$

onde $\epsilon \in[0,1)$ é um parâmetro que controla a deformação da fronteira, $p$ é um número inteiro e $\theta^{\prime}(t)=\theta+\Omega t$, onde $\Omega$ define a frequência de giro e $t$ é o instante de colisão. A dependência temporal no sistema é introduzida ao fazermos a transformação em $\theta$, 
que produz a rotação, por isso, se $\Omega=0$ a fronteira é estática e os resultados para o caso estático podem ser recuperados (93). Para $\Omega \neq 0$ a fronteira gira com velocidade angular $\Omega$. As componentes cartesianas da fronteira na posição $\left(\theta_{n}, t_{n}\right)$ são dadas por

$$
\begin{aligned}
& X\left(\theta_{n}, t_{n}\right)=\left[1+\epsilon \cos \left[p \theta^{\prime}(t)\right]\right] \cos \left(\theta_{n}\right), \\
& Y\left(\theta_{n}, t_{n}\right)=\left[1+\epsilon \cos \left[p \theta^{\prime}(t)\right]\right] \sin \left(\theta_{n}\right) .
\end{aligned}
$$

Começando com uma condição inicial $\left(\theta_{n}, \alpha_{n},\left|V_{n}\right|, t_{n}\right)$, o ângulo entre a reta tangente na fronteira e o eixo horizontal no ponto $X\left(\theta_{n}\right)$ e $Y\left(\theta_{n}\right)$ é dado por $\phi_{n}=$ $\arctan \left[Y^{\prime}\left(\theta_{n}\right) / X^{\prime}\left(\theta_{n}\right)\right]$, onde $X^{\prime}=d X / d \theta$ e $Y^{\prime}=d Y / d \theta$. Como não existem forças atuando na partícula durante o voo, a trajetória entre os choques é retilínea. Para $t>t_{n}$ a posição da partícula como função do tempo é dada por

$$
\begin{aligned}
X_{p}(t) & =X\left(\theta_{n}, t_{n}\right)+\left|\vec{V}_{n}\right| \cos \left(\alpha_{n}+\phi_{n}\right)\left(t-t_{n}\right), \\
Y_{p}(t) & =Y\left(\theta_{n}, t_{n}\right)+\left|\vec{V}_{n}\right| \sin \left(\alpha_{n}+\phi_{n}\right)\left(t-t_{n}\right) .
\end{aligned}
$$

Uma vez que a posição da partícula em função do tempo é conhecida, sua distância medida em relação à origem do sistema de coordenadas é dada por $R_{p}\left(\theta_{p}, t_{n}\right)=$ $\sqrt{X_{p}^{2}(t)+Y_{p}^{2}(t)}$ e $\theta_{p}=\arctan \left[Y_{p}(t) / X_{p}(t)\right]$. A posição angular na próxima colisão da partícula com a fronteira, isto é $\theta_{n+1}$, é numericamente obtida resolvendo a seguinte equação $R_{b}(\theta, t)=R_{p}(\theta, t)$. O tempo é obtido por

$$
t_{n+1}=t_{n}+\frac{\sqrt{\Delta X^{2}+\Delta Y^{2}}}{\left|\vec{V}_{n}\right|}
$$

onde $\Delta X=X\left(\theta_{n+1}\right)-X\left(\theta_{n}\right)$ e $\Delta Y=Y\left(\theta_{n+1}\right)-Y\left(\theta_{n}\right)$. Para obter a nova velocidade nós devemos observar que o referencial da fronteira está se movendo. Então, no instante de colisão, a seguinte condição deve ser satisfeita

$$
\begin{aligned}
& \vec{V}_{n+1}^{\prime} \cdot \vec{T}_{n+1}=\vec{V}_{n}^{\prime} \cdot \vec{T}_{n+1}, \\
& \vec{V}_{n+1}^{\prime} \cdot \vec{N}_{n+1}=-\vec{V}_{n}^{\prime} \cdot \vec{N}_{n+1},
\end{aligned}
$$

onde $\vec{T}$ e $\vec{N}$ são os vetores unitários tangente e normal e $\vec{V}^{\prime}$ indica a velocidade da partícula medida no referencial móvel. As velocidades das componentes da partícula são dadas por

$$
\begin{aligned}
\vec{V}_{n+1} \cdot \vec{T}_{n+1} & =\left|\vec{V}_{n}\right|\left[\cos \left(\alpha_{n}+\phi_{n}\right) \cos \left(\phi_{n+1}\right)\right]+ \\
& +\left|\vec{V}_{n}\right|\left[\sin \left(\alpha_{n}+\phi_{n}\right) \sin \left(\phi_{n+1}\right)\right] \\
\vec{V}_{n+1} \cdot \vec{N}_{n+1} & =-\left|\vec{V}_{n}\right|\left[\sin \left(\alpha_{n}+\phi_{n}\right) \cos \left(\phi_{n+1}\right)\right]+ \\
& +\left|\vec{V}_{n}\right|\left[\cos \left(\alpha_{n}+\phi_{n}\right) \sin \left(\phi_{n+1}\right)\right]+ \\
& +2 \vec{V}_{b}\left(t_{n+1}\right) \cdot \vec{N}_{n+1},
\end{aligned}
$$

onde $\vec{V}_{b}\left(t_{n+1}\right)$ é a velocidade da fronteira e $\vec{V}_{b}\left(t_{n+1}\right) \cdot \vec{N}_{n+1}$ é escrito como

$$
\begin{aligned}
& \vec{V}_{b}\left(t_{n+1}\right) \cdot \vec{N}_{n+1}=-\epsilon p \Omega \sin \left[p \theta^{\prime}\left(t_{n+1}\right)\right] \times \\
& \times\left[-\cos \left(\theta_{n+1}\right) \sin \left(\phi_{n+1}\right)+\sin \left(\theta_{n+1}\right) \cos \left(\phi_{n+1}\right)\right] .
\end{aligned}
$$




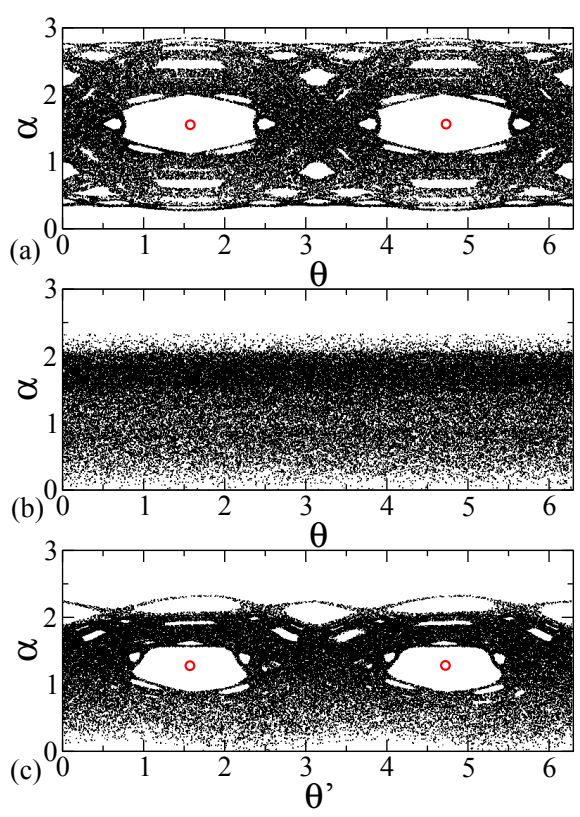

Figura 5.2: Gráfico mostrando: (a) $\alpha \times \theta$ para $\Omega=0$; (b) $\alpha \times \theta$ para $\Omega=1$ e; (c) $\alpha \times(\theta+\Omega t)(\bmod 2 \pi)$. Os parâmetros usados foram $\epsilon=0,1$ e a condição inicial foi $V_{0}=3$, $\theta_{0}=\pi, t_{0}=0$ e $\alpha_{0}=\pi / 2-0,1$. A órbita foi evoluída até $2 \times 10^{5}$ colisões com a fronteira.

Portanto, a velocidade da partícula na colisão $(n+1)$ é dada por

$$
\left|\vec{V}_{n+1}\right|=\sqrt{\left(\vec{V}_{n+1} \cdot \vec{T}_{n+1}\right)^{2}+\left(\vec{V}_{n+1} \cdot \vec{N}_{n+1}\right)^{2}} .
$$

Finalmente, $\alpha_{n+1}$ é obtido por

$$
\alpha_{n+1}=\arctan \left[\frac{\vec{V}_{n+1} \cdot \vec{N}_{n+1}}{\vec{V}_{n+1} \cdot \vec{T}_{n+1}}\right] .
$$

\subsection{Resultados numéricos}

Sabe-se que para valores fixos de $p$ e $\epsilon$ existe um parâmetro crítico onde a curvatura da fronteira muda de positiva para neutra e então localmente negativa (93). Tal valor é dado por

$$
\epsilon_{c}=\frac{1}{1+p^{2}}, \quad p \geq 1 .
$$

Se $\epsilon<\epsilon_{c}$, o espaço de fases para o caso estático é misto, contendo ilhas periódicas, mares de caos e curvas invariantes do tipo spanning. Se $\epsilon>\epsilon_{c}$ as curvas invariantes são destruídas, isto ocorre pois algumas regiões da fronteira tem curvatura não convexa. 


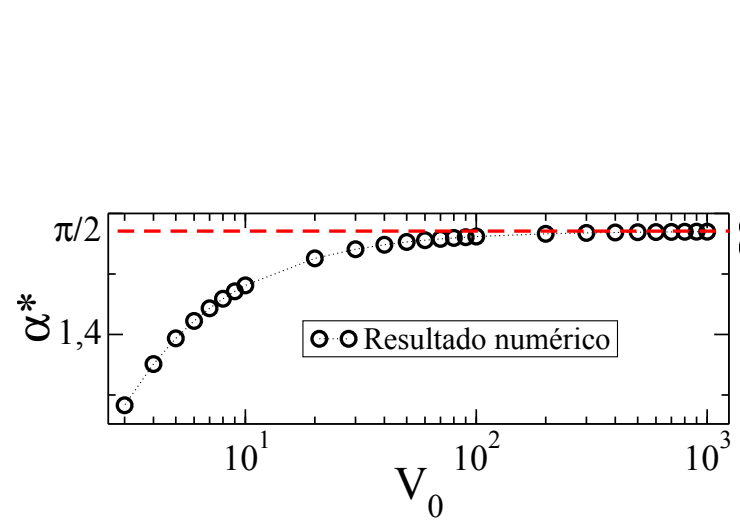

Figura 5.3: Gráfico da coordenada angular do ponto fixo de período dois para diferentes valores de $V_{0}$ e usando $\epsilon=0,1$ e $\Omega=1$. A linha pontilhada é dada por $\alpha^{*}=\pi / 2$.

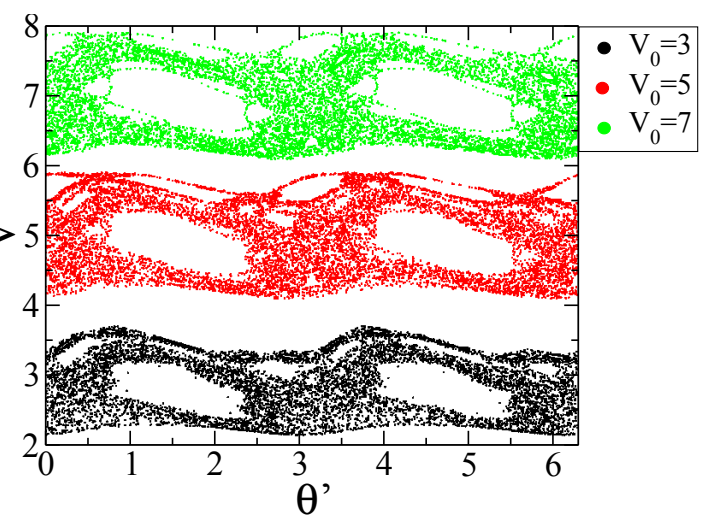

Figura 5.4: Gráfico de $V$ vs. $\theta^{\prime}$ para diferentes valores de $V_{0}$ e considerando $\epsilon=0,1$, $\Omega=1, \alpha_{0}=\pi / 2-0,1$ e $\theta_{0}=0,16$. Cada órbita foi iterada $10^{4}$ vezes.

Para todas as simulações consideraremos $p=2$, o que nos leva a concluir que $\epsilon_{c}=0,2$. Dividiremos os resultados em dois casos: (i) $\epsilon\left\langle\epsilon_{c}\right.$ e (ii) $\epsilon>\epsilon_{c}$. Para o caso (i), mostraremos que o fenômeno de aceleração de Fermi não é observado para a combinação de parâmetros de controle e condições iniciais escolhidas. Para o caso (ii), o crescimento ilimitado de energia é observado, como previsto pela conjectura LRA.

\subsubsection{Caso (i): $\epsilon<\epsilon_{c}$}

A figura 5.2 mostra o comportamento de uma órbita iterada $n=2 \times 10^{5}$ vezes, onde a condição inicial usada foi $\theta_{0}=\pi, \alpha_{0}=\pi / 2-0,1, V_{0}=3, t_{0}=0$ e em (a) $\Omega=0$. Vemos um mar caótico e curvas invariantes do tipo spanning, além de diversas ilhas periódicas. Destacamos com círculos vermelhos uma órbita de período dois. A figura 5.2(b) mostra a órbita considerando $\Omega=1$. Como podemos ver as estruturas presentes na figura 5.2(a) não são mais observadas, entretanto, aplicando a transformação $\theta^{\prime} \rightarrow \theta+\Omega t$ uma estrutura similar ao item (a) é vista, como mostrado na figura 5.2(c). Tal transformação foi proposta pois para cada ponto $(X, Y)$ faremos uma rotação no sentido contrário do giro, desta forma a fronteira fica estática e a partícula se move com arcos que serão descritos a seguir. A posição e forma das ilhas periódicas muda e depende da velocidade inicial adotada. Se a velocidade da partícula é maior do que a velocidade da fronteira, a partícula experimenta diversas colisões em um pequeno intervalo de tempo, e no limite de $V \rightarrow \infty$ o espaço de fases para o caso estático é recuperado. O ângulo $\alpha^{*}$ para o ponto fixo elíptico de período dois (que é representado pelos círculos vermelhos das figuras 5.2(a) e 5.2(c)) em função de $V_{0}$ é mostrado na figura 5.3, onde no limite $V_{0} \rightarrow \infty$ temos que $\alpha \rightarrow \pi / 2$. A figura 5.4 mostra o comportamento de $V \times \theta^{\prime}$ para $\epsilon=0,1, \alpha_{0}=\pi / 2-0,1$ e $\theta_{0}=0,16$ e considerando diferentes valores de $V_{0}$ iterados até $10^{4}$ vezes. É importante notar que para diferentes valores de $V_{0}$, as estruturas 

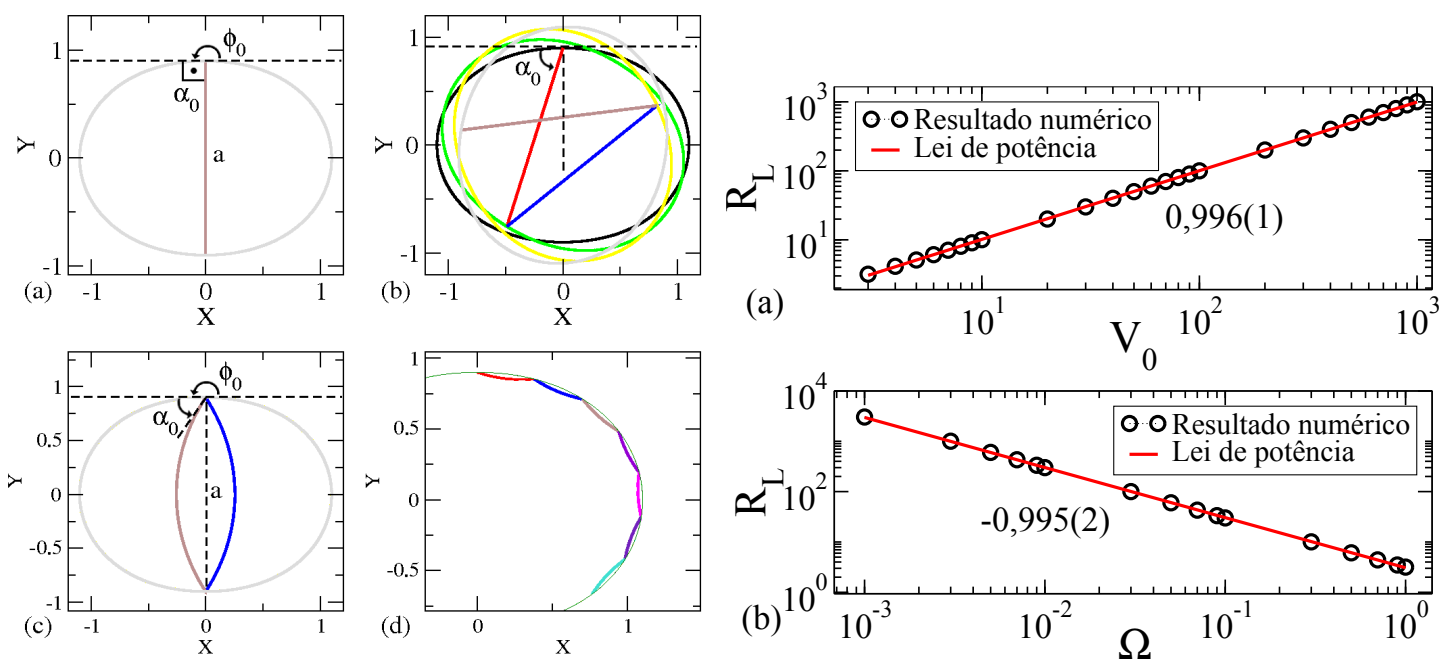

Figura 5.5: Gráfico de uma órbita usando $V_{0}=3, \theta_{0}=\pi / 2$ e $t_{0}=0$ para: (a) $\Omega=0$ e $\alpha_{0}=\pi / 2$ (ponto fixo de período dois); (b) $\Omega=1$ e $\alpha_{0}=1,28312$ (outro exemplo de trajetória em um ponto fixo); (c) O mesmo mostrado no item (b) considerando a reescala $\theta \rightarrow \theta+\Omega t$; (d) Um exemplo de saltos curtos ao longo da fronteira para $\Omega=1$ e $\alpha_{0}=2,9$ (correspondendo a uma órbita rotator).

Figura 5.6: (a) Gráfico de $R_{L}$ em função de $V_{0}$. Depois de um ajuste em lei de potência o expoente obtido foi $0,996(1)$;

(b) $R_{L}$ vs. $\Omega$ e o expoente obtido foi $-0,995(2)$.

observadas no plano $V \times \theta^{\prime}$ são similares (ver figura 5.4). A evolução da dinâmica leva a velocidade da partícula a variar dentro de um certo limite, produzindo estruturas que aparentemente não são conectadas umas às outras. Portanto, uma órbita em tal região nunca escapa desta região, consequentemente levando a um crescimento finito de energia.

A figura 5.5(a) mostra uma trajetória para $V_{0}=3, \alpha_{0}=\theta_{0}=\pi / 2$ e $\Omega=0$. Para tal caso, a partícula apenas colide em uma região diametral do bilhar. Considerando o caso de $\Omega=1$ e $V_{0}=3$, para atingir a mesma posição, o valor de $\alpha_{0}$ deve mudar porque a geometria está rodando [ver figura 5.5(b)]. Entretanto, considerando a mesma transformação feita antes para $\theta$, isto é, $\theta^{\prime} \rightarrow \theta+\Omega t$, isto pode ser interpretado como se a fronteira ficasse na mesma posição e a partícula se movesse com trajetórias fazendo arcos, como mostrado na figura 5.5(c). Tais trajetórias são chamadas círculos de Larmor $(94,95)$. Para uma partícula clássica de massa $m$ e carga $q$ movendo com velocidade constante $v$ em uma região plana, se um campo magnético constante e uniforme $B$ é direcionado perpendicularmente ao plano de movimento, as trajetórias consistem de uma série de arcos circulares com raio de Larmor que é definido como

$$
R_{L}=m v /(q B),
$$

onde $R$ é proporcional à velocidade $v$ e inversamente proporcional ao campo magnético 


\subsection{Resultados numéricos}
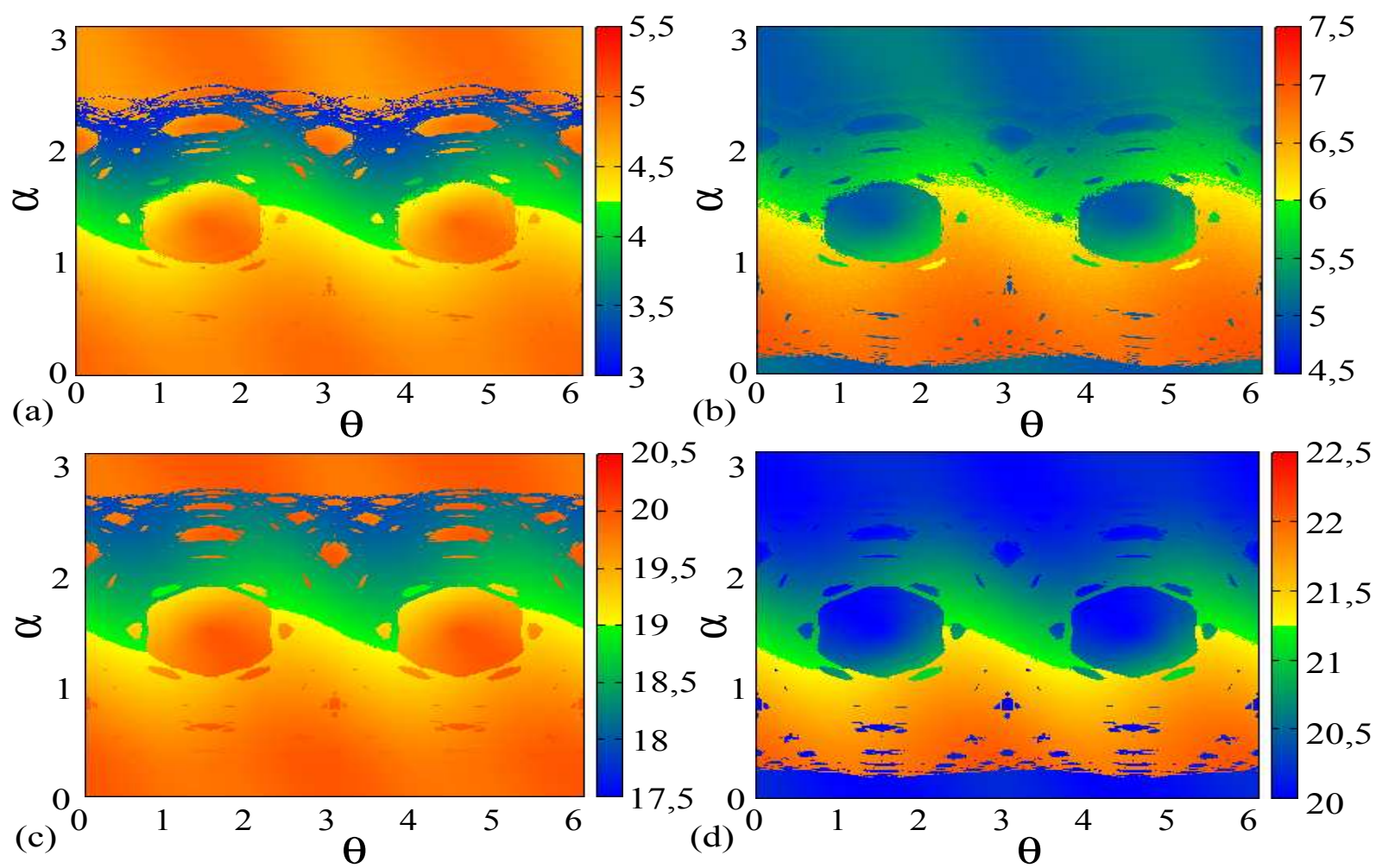

Figura 5.7: Diagrama de $\alpha \times \theta$ onde as cores representam as mínimas (a,c) e máximas (b,d) velocidades ao longo da órbita. O tempo inicial foi $t_{0}=\mathrm{e}$ : $(\mathrm{a}, \mathrm{c}) V_{0}=5$ e $(\mathrm{b}, \mathrm{d})$ $V_{0}=20$.

B. A figura 5.5(c) mostra um exemplo de tal órbita diametral e fechada. Seguindo a Ref. (94), concluímos que $R_{L}=a /\left[2 \cos \left(\alpha^{*}\right)\right]$, onde $a$ é a distância entre dois pontos da colisão com a fronteira. Para $\epsilon=0,1$ e após diversas simulações, obtemos $a \cong 1,8$. A figura 5.6(a) mostra o comportamento de $R_{L}$ em função de $V_{0}$, e após um ajuste em lei de potência obtivemos um expoente $0,996(1) \cong 1$, portanto $R_{L} \propto V_{0}$. Por outro lado, variando $\Omega \in\left[10^{-3}, 1\right]$ obtemos que $R_{L} \propto \Omega^{-1}$ como mostrado na figura 5.6(b). Já na figura $5.5(\mathrm{~d})$ mostramos uma órbita rotator ${ }^{1}$, que é um regime de curtos saltos ao longo da fronteira (94). Concluímos que $R_{L} \propto V_{0} / \Omega$ e comparando com a Eq. (5.15), temos que a velocidade $v$ faz o papel da velocidade $V_{0}$ do nosso bilhar e o campo magnético $B$ tem como equivalente o $\Omega$. Portanto, a dependência temporal do tipo giro de alguma forma faz com que a partícula se mova como se estivesse experimentando o efeito de um campo magnético constante.

Para checar a influência dos correspondentes valores mínimos e máximos da velocidade ao longo da órbita em função da condição inicial, a figura 5.7 mostra um gráfico das condições iniciais $\alpha \times \theta$ onde as cores caracterizam os limites alcançados por $V$. Para

\footnotetext{
$\theta$.

${ }^{1}$ Uma trajetória rotator é localizada próxima da borda da fronteira e passa por todos os valores de
} 


\section{BILHAR OVÓIDE GIRANTE}
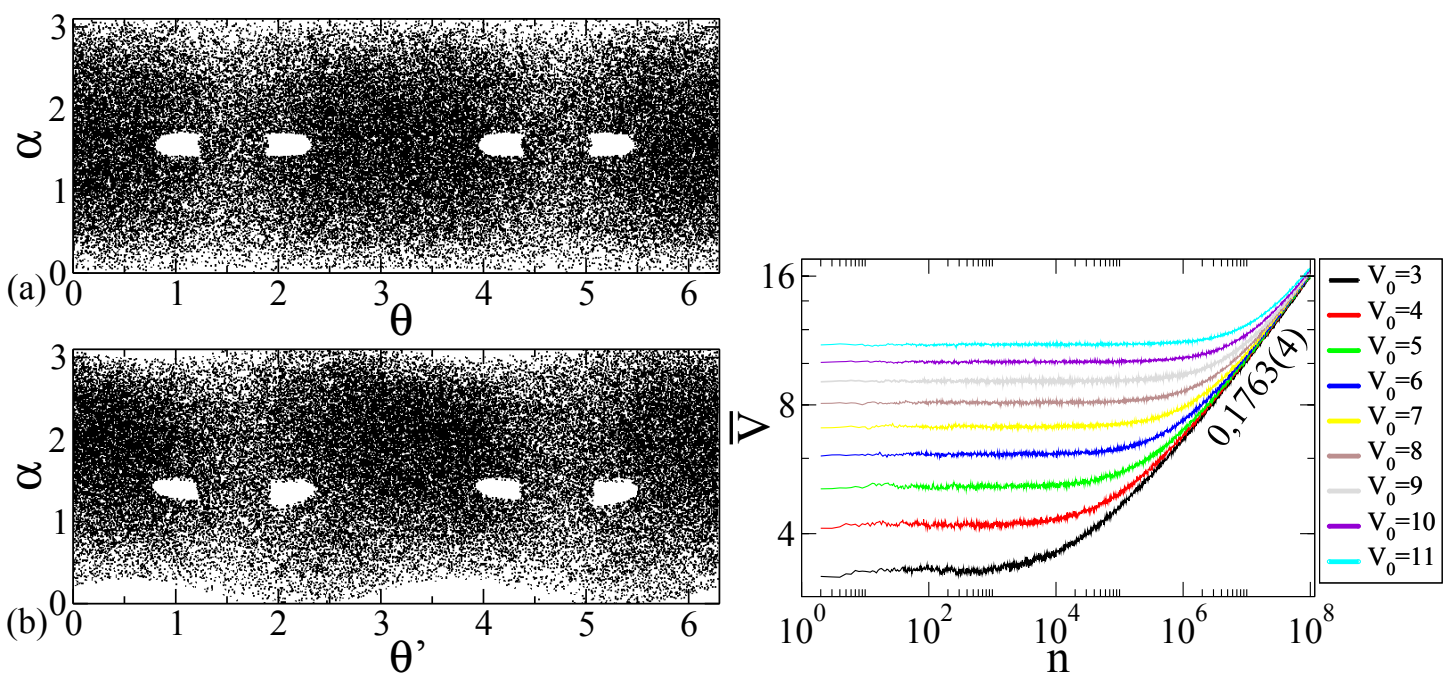

Figura 5.8: Gráfico de: (a) $\alpha \times \theta$ para $\Omega=0$ e; (b) $\alpha \times \theta^{\prime}$ para $\Omega=1$. Os parâmetros usados foram $\epsilon=0,3$ para a condição inicial $\alpha_{0}=\pi / 2-0,1, \theta_{0}=\pi$, $t_{0}=0$ e $V_{0}=3$.

Figura 5.9: Gráfico de $\bar{V}$ em função de $n$ usando 300 diferentes condições iniciais escolhidas aleatoriamente $\left(t_{0} \in(0,2 \pi], \theta_{0} \in\right.$ $(0,2 \pi]$ e $\left.\alpha_{0} \in(0, \pi]\right)$ para cada $V_{0}$. As órbitas foram iteradas $10^{8}$ vezes.

as figuras $5.7(\mathrm{a}, \mathrm{c})$ as cores mostram a velocidade mínima ao longo da série variando entre 3 e no máximo 5,5 para uma velocidade inicial de $V_{0}=5$ enquanto as figuras $5.7(\mathrm{~b}, \mathrm{~d})$ correspondem as velocidades máximas até um máximo de $n=10^{6}$ colisões com a fronteira. Como podemos ver na figura 5.7, as velocidades são maiores para $\alpha$ aproximadamente menor do que $\pi / 2$, nestes casos temos que a velocidade de giro da partícula coincide com o sentido de giro da fronteira. As velocidades menores se encontram normalmente em $\alpha>2$, que seria o sentido oposto ao de giro da fronteira.

\subsubsection{Caso (ii): $\epsilon>\epsilon_{c}$}

Consideraremos o caso em que $\epsilon=0,3>\epsilon_{c}$. Para este valor de $\epsilon$ existem duas regiões de curvaturas não positivas (para o caso estático), uma próxima de $\theta=\pi / 2$ e outra em $\theta=3 \pi / 2$. A figura 5.8(a) mostra o esboço de uma órbita para o caso independente do tempo onde $\Omega=0$ e considerando a condição inicial $\alpha_{0}=\pi / 2-0,1, \theta_{0}=\pi$, $t_{0}=0$ e $V_{0}=3$. Para tal caso, podemos ver que as curvas invariantes do tipo spanning são destruídas, porém ilhas periódicas e um extenso mar caótico ainda são observados. A figura 5.8(b) também mostra uma órbita porém agora considerando $\Omega=1$, onde utilizamos a transformação $\theta^{\prime}=\theta+\Omega t$. Vemos que os espaços de fases são parecidos, porém a forma das ilhas de período 2 são alteradas na medida em que variamos o valor de $\Omega$.

Estudaremos o comportamento da velocidade média em função do número de colisões com a fronteira para um conjunto de condições iniciais. Fixaremos a condição inicial $V_{0}$ enquanto os outros observáveis, $\theta_{0} \in(0,2 \pi], \alpha_{0} \in(0, \pi]$ e $t_{0} \in(0,2 \pi]$ são 

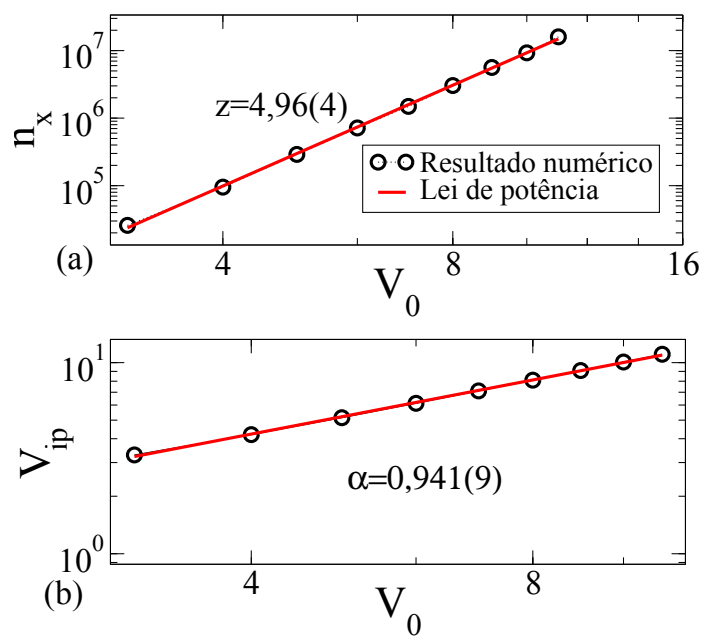

Figura 5.10: (a) Gráfico de $n_{x}$ vs. $V_{0}$. Um ajuste em lei de potência fornece o expoente $z=4,96(4)$; (b) Gráfico da velocidade inicial do plato $V_{i p}$ em função de $V_{0}$. A inclinação encontrado após um ajuste em lei potência foi $\alpha=0,941(9)$.

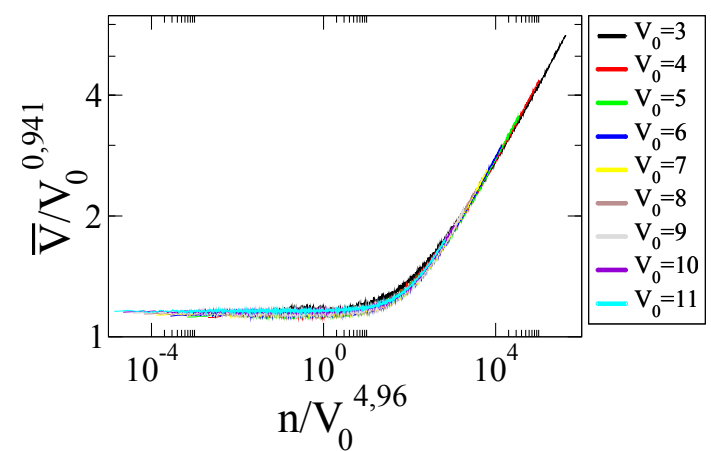

Figura 5.11: Reescala de todas as curvas mostradas na figura 5.9 em uma única e universal curva, onde as transformações nos eixos usadas foram $\left(V \rightarrow V / V_{0}^{0,941}\right)$ e $\left(n \rightarrow n / V_{0}^{4,96}\right)$.

escolhidos aleatoriamente. Avaliaremos a velocidade média sobre a órbita para uma condição inicial, que é definida como $V_{i}=\frac{1}{n+1} \sum_{j=0}^{n} V_{i, j}$, onde o índice $i$ corresponde a uma amostra de um conjunto de condições iniciais e a velocidade média sobre o conjunto é escrita como $\bar{V}=\frac{1}{M} \sum_{i=1}^{M} V_{i}$, onde $M=300$ simboliza o número de diferentes condições iniciais. A figura 5.9 mostra o comportamento da velocidade média em função de $n$ para nove diferentes velocidades iniciais. Para $n$ pequeno podemos ver que as curvas permanecem constantes, e depois de um crossover $n_{x}$ todas iniciam um crescimento com expoente $\beta=0,1763(4) \cong 1 / 6$. O valor numérico encontrado é aproximadamente o mesmo valor que fora encontrado em bilhares com perturbação temporal do tipo breathing ${ }^{1}(96,97)$. A figura 5.10(a) mostra $n_{x}$ vs $\mathrm{V}_{0}$ e após um ajuste em lei de potência o expoente obtido foi $z=4,96(4)$. Considerando a velocidade do platô inicial em função de $V_{0}$, encontramos um expoente $\alpha=0,941$ (9) (figura 5.10(b)). Usando estes resultados, podemos colapsar todas as curvas mostradas na figura 5.9 fazendo uma transformação $\bar{V} \rightarrow \bar{V} / V_{0}^{0,941}$ e $n \rightarrow n / V_{0}^{4,96}$, como feito na figura 5.11. O regime de crescimento da velocidade é uma confirmação de que a aceleração de Fermi está agindo nas partículas, onde este é um caso diferente do $\epsilon<\epsilon_{c}$, em que não vemos um aparente crescimento ilimitado de energia.

\footnotetext{
${ }^{1}$ Uma tradução para bilhares do tipo breathing seria bilhares que "respiram" ou que pulsam.
} 


\section{BILHAR OVÓIDE GIRANTE}

\subsection{Conclusões}

Estudamos algumas propriedades dinâmicas de um bilhar oval com perturbação temporal do tipo giro. Mostramos que o modelo é descrito em termos de um mapeamento não-linear e quadridimensional. Para o caso onde a curvatura da fronteira é estritamente positiva $\left(\epsilon<\epsilon_{c}\right)$ mostramos que o sistema não apresenta aceleração de Fermi e isso é aparentemente uma contradição da conjectura LRA para bilhares bidimensionais. Mostramos que a posição angular pode ser reescalada e as partículas se movem através de círculos de Larmor, com isso, temos uma conexão com bilhares magnéticos. Para o caso onde a fronteira tem curvatura negativa $\left(\epsilon>\epsilon_{c}\right)$, mostramos que o fenômeno de crescimento ilimitado de energia é observado e a velocidade média é invariante de escala em relação a velocidade inicial. Os resultados apresentados neste capítulo foram publicados na Communications in Nonlinear Science $\& 5$ Numerical Simulation (35). 


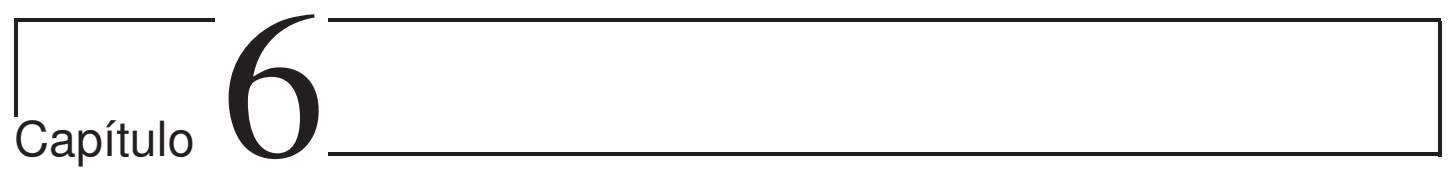

\section{Conclusões finais}

Nesta tese estudamos algumas propriedades dinâmicas e de escape de partículas clássicas não interagentes considerando diferentes sistemas. Vimos alguns exemplos de invariância de escala que ocorrem, por exemplo, na energia média ou desvio da energia média; no histograma do número de partículas que atingem certa altura $h$ no espaço de fases; na probabilidade de sobrevivência; no coeficiente de difusão. Invariâncias de escala são importantes pois mostram que após reescalas apropriadas, alguns observáveis médios no espaço de fases têm comportamentos universais. Detalhamos a forma de obter os mapeamentos, e pudemos observar que os sistemas descritos aqui, em sua maioria, apresentam uma estrutura mista no espaço de fases, contendo ilhas periódicas, caos e curvas invariantes do tipo spanning. Expoentes de Lyapunov foram utilizados para caracterizar os sistemas.

No capítulo 2 estudamos um guia de ondas senoidalmente corrugado, onde tal modelo considera as trajetórias de um raio de luz que sofre reflexões entre um espelho plano e outro liso. Obtivemos resultados tanto para a versão completa quanto simplificada do mapeamento. Como mencionado, a versão simplificada do modelo não nos traz informações sobre reflexões múltiplas com a fronteira corrugada, porém facilita nossas análises analíticas. É interessante salientar que neste problema damos uma motivação física para o escape de partículas, onde utilizando as equações de Fresnel conseguimos mostrar em quais situações temos o escape do raio de luz ao considerar meios materiais com diferentes índices de refração. Os resultados apresentados nesta seção foram recentemente publicados no Journal of Physics A, Mathematical and Theoretical (28), Physics Letters A (29) e Communications in Nonlinear Science $\& 5$ Numerical Simulation (30).

O capítulo 3 mostrou uma família de mapas Hamiltonianos bidimensionais que recupera diversos modelos. Após uma conexão com o mapa padrão, obtivemos analiticamente os expoentes de escala. Coeficientes de difusão foram obtidos considerando histogramas de frequência para o número de partículas que atingem uma certa altura no espaço de fases. Também mostramos que os coeficientes de difusão são invariantes de escala após reescalas apropriadas. Os resultados deste capítulo foram publicados, 


\section{CONCLUSÕES FINAIS}

conforme mostrado na Ref. (31).

O capítulo 4 mostrou partículas confinadas em uma caixa com potenciais infinitos nas bordas e contendo em seu interior um poço de potencial dependente periodicamente do tempo. Tal modelo mostra a dinâmica de um conjunto de partículas clássicas que sofre a influência de diferentes potenciais. Dependendo da região que a partícula se encontra e a fase do potencial móvel, é possível haver ganhos ou perdas de energia cinética. Os resultados foram publicados em $(32,33,34)$. Aqui mostramos as expressões dos pontos fixos elípticos e hiperbólicos de período um. Mostramos pela primeira vez na literatura que o histograma do número de partículas que escapam em uma dada iterada $n$ é invariante de escala (após reescalas apropriadas) ao considerarmos a posição do buraco proporcional à posição da primeira curva invariante spanning.

Já o capítulo 5 apresentou um bilhar ovóide com dependência temporal introduzida através de giro. Para certas condições observamos que este não apresenta um crescimento ilimitado de energia (aceleração de Fermi), desta forma sendo um possível contraexemplo da conjectura LRA. Neste bilhar as partículas se movem através de círculos de Larmor, com isso, temos uma conexão com bilhares magnéticos. Para o caso em que temos curvatura negativa $\left(\epsilon<\epsilon_{c}\right)$, mostramos que o fenômeno de crescimento ilimitado de energia é observado e a velocidade média das partículas é invariante de escala em relação a velocidade inicial. Os resultados do bilhar ovóide girante foram publicados recentemente (35).

Esta tese foi um resumo de 8 artigos que foram publicados em revistas internacionais. Todos os resultados que foram apresentados nesta tese são novos, originais e deverão contribuir para o avanço do conhecimento na área de sistemas dinâmicos. 


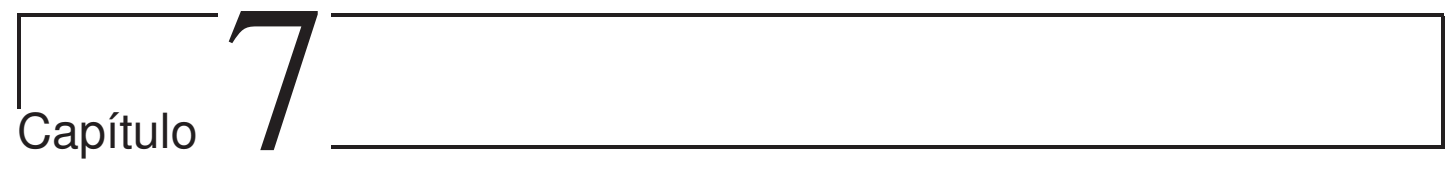

\section{Perspectivas futuras}

Durante o tempo de doutoramento, tive a oportunidade de aprimorar os meus conhecimentos em sistemas dinâmicos, caos, bilhares, entre outros. Pude submeter para publicação alguns artigos, adiantar outros e além de contar com alguns aceitos para publicação ou até mesmo publicados. Além dos oito artigos citados nesta tese, cujas referências são $(28,29,30,31,32,33,34,35)$, outro trabalho também foi publicado em 2012, desta vez na International Journal of Bifurcation and Chaos in Applied Sciences and Engineering (11).

Os trabalhos abaixo foram submetidos para publicação:

- 'Phase space properties and chaotic transport for a particle moving in a time dependent step potential well', submetido para publicação na Applied Mathematics and Computation (98);

- 'Circular, elliptic and oval billiards in a gravitational field', submetido para publicação na Communications in Nonlinear Science and Numerical Simulation (99);

- 'Statistical properties for a dissipative model of relativistic particles in a wave packet: a parameter space investigation', submetido para publicação na Applied Mathematics and Computation (100);

- 'Transport and dynamical properties for a bouncing ball model with regular and stochastic perturbations', submetido para publicação na Physical Review E (101);

No meu terceiro ano de doutorado realizei um estágio em pesquisa com bolsa BEPE da FAPESP (processo $n^{\circ}$ 2012/18962-0). Fiquei por um período de 6 meses no grupo do prof. Carl Dettmann em Bristol (Reino Unido). Tal visita rendeu alguns trabalhos que estão em processo de finalização, publicados ou submetidos.

Após a visita à Inglaterra, finalizei alguns artigos, dediquei-me à escrita da tese e preparação para à defesa.

Gostaria de deixar aqui meus agradecimentos ao co-orientador Prof. Dr. Iberê L. Caldas pelas discussões, por toda a contribuição dada ao longo do projeto de doutorado e por toda a paciência. Como citado nas referências, nossa parceria rendeu 2 artigos, sendo 1 deles submetido (98) e outro publicado(34), além de outros trabalhos que estão 


\section{PERSPECTIVAS FUTURAS}

em processo de finalização.

Pretendo iniciar um Pós-Doutorado no início de 2014, onde continuarei com os meus estudos em sistemas dinâmicos e bilhares. O novo projeto de pesquisa deverá contar com a colaboração de diferentes grupos de pesquisa cujos pesquisadores responsáveis são: (i) Prof. Iberê L. Caldas (USP / São Paulo); (ii) Prof. Leonid Bunimovich (Georgia Institute of Technology / USA); (iii) Prof. Eduardo Altmann (Max-Planck-Institute / Dresden); (iv) Prof. Dettmann (University of Bristol / Inglaterra); (v) Prof. Edson Denis Leonel (UNESP - Rio Claro/SP); (vi) Prof. Jose Antonio Mendez-Bermudez (Universidad Autonoma de Puebla / México). 


\section{Lista de Figuras}

2.1 Esquema ilustrativo do guia de ondas e dos ângulos envolvidos em sua descrição. . . . . . . . . . . . . . . . . . . 9

2.2 Espaço de fases para: (a) $\delta=0,1$; (b) $\delta=0,01$. Podemos ver que o tamanho ocupado pelo espaço de fases depende do parâmetro $\delta$. . . . 10

2.3 (a) Gráfico de $P \times n_{s r}$ para: $\delta=10^{-3}, \delta=10^{-2}$ e $\delta=10^{-1}$. (b) Depois de uma reescala $\left(n_{s r} \rightarrow n_{s r} / \delta^{0,252}\right)$, todas as curvas de (a) colapsam uma sobre as outras em uma única curva universal. . . . . . . . . . . . . . 11

2.4 Gráfico de $n_{s r}^{\prime} \times \delta$. Um ajuste em lei de potência nos fornece como

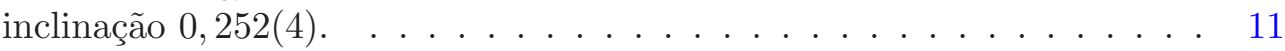

2.5 Gráfico de $\Delta \omega / \omega \times M$ para: (a) $n=10$ colisões; (b) $n=10000$ colisões. Mesmos comportamentos foram obtidos para diversos valores de $n \in$ $[10,10000] \ldots \ldots \ldots \ldots \ldots \ldots \ldots \ldots$

2.6 Gráfico de $\omega \times n$ para os parâmetros $\delta=2 \times 10^{-4}, \delta=5 \times 10^{-4} \mathrm{e}$ $\delta=3 \times 10^{-3}$; (b) Depois de uma reescala $\left(n \rightarrow n \delta^{2+z}\right.$ e $\left.\omega \rightarrow \omega / \delta^{\alpha}\right)$ todas as curvas de (a) colapsam em uma única curva universal. Usamos um conjunto de $M=1000$ condições iniciais. . . . . . . . . . . . 12

2.7 Para $M=1000$ : (a) Gráfico de $\omega_{\text {sat }} \times \delta$, onde o expoente encontrado foi $\alpha=0,508(4)$; (b) Resultados para $n_{x} \times \delta$, onde $z=-0,956(9) \ldots \ldots 13$

2.8 (a) Curvas de reflectância para raios $s$-polarizado (curva preta contínua) e p-polarizado (linha tracejada vermelha) em função do ângulo de incidência $\theta_{i}$. Foi considerado $n_{21}=n_{2} / n_{1}=1 / 1,5$; (b) Terceira potência da reflectância em função do ângulo de incidência $\theta_{i}$ para raios $s$-polarizado (curva continua preta) e $p$-polarizado (linha tracejada vermelha). . . . .

2.9 Comportamento da primeira curva invariante spanning $\left(A_{\text {inv }}\right)$ em função do parâmetro $\delta$. Um ajuste em lei de potência fornece $\left(\pi / 2-A_{\text {inv }}\right) \propto \delta^{\alpha}$ $\operatorname{com} \alpha=0,495(5) \ldots \ldots \ldots \ldots \ldots \ldots \ldots$

2.10 (a) Gráfico do histograma relativo de órbitas que escaparam para $c_{p}=$ 0,3 e três diferentes valores do parâmetro $\delta$. (b) Depois de uma reescala no eixo horizontal $\left(n \rightarrow n / \delta^{-0,963}\right)$ e vertical $\left(H \rightarrow H / \delta^{0,953}\right)$ todas as curvas mostradas em (a) colapsam em uma única curva universal. . . . . 
2.11 (a) Gráfico de $n_{p}$ em função de $\delta$ para diferentes valores de $c_{p}$ e considerando o escape em ambas partes lisa e corrugada do bilhar. Um ajuste em lei de potência fornece $z=-0,963(4) \cong-1$; (b) Gráfico de $H_{\max }$ em função de $\delta$ para três diferentes valores de $c_{p}$. O expoente obtido foi $\gamma=0,953(1) \cong 1$; (c) Gráfico de $n_{p}$ em função de $c_{p}$ para $\delta=10^{-2}$ e $\delta=10^{-3}$, onde o expoente médio obtido foi $u=-2,05(3) . \quad . \quad 17$

2.12 Índice de refração $\left(n_{1}\right)$ em função de $\delta$, onde destacamos alguns materiais. Os dados foram obtidos da Ref. (47). . . . . . . . . . . . . 18

2.13 Evolução de $10^{4}$ condições iniciais diferentes iteradas: (a) 5 e; (b) 100 vezes. A curva preta tracejada representa o escape na região plana do bilhar enquanto a linha vermelha mostra o escape na parte corrugada do guia de ondas. . . . . . . . . . . . . . . .

2.14 Espaço de fases para o mapeamento (2.16) usando o parâmetro de con-

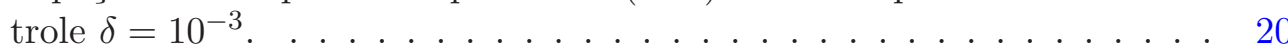

2.15 Expoente de Lyapunov positivo como função do parâmetro de controle $\delta$. 21

2.16 Gráfico do menor valor de $\gamma$ na curva invariante spanning como função de $\delta$ junto com o resultado mostrado na Eq. (2.19). Um ajuste em lei de potência nos fornece $\gamma \propto \delta^{0,505(2)}$

2.17 (a) Histograma para diferentes $\gamma_{c}$ e diferentes valores de $\delta$. (b) Colapso de todas as curvas mostradas no item (a) em um único e universal gráfico após uma reescala $n \rightarrow n /\left(\gamma_{c}^{u} \delta^{\bar{\zeta}}\right) \ldots \ldots \ldots \ldots$

2.18 (a) Gráfico de $n_{p}$ vs $\gamma_{c}$. Após um ajuste em lei de potência o expoente obtido foi $u=1,97(1)$. (b) Gráfico de $n_{p} \times \delta$ e os respectivos ajustes em lei de potência para $\gamma_{c}=20 \% \gamma_{i n v} \operatorname{com} \zeta=-0,950(8) ; \gamma_{c}=40 \% \gamma_{i n v}$ com $\zeta=-0,951(6)$; e finalmente $\gamma_{c}=70 \% \gamma_{i n v} \operatorname{com} \zeta=-0,97(2) . \ldots 23$

3.1 Gráfico de $P^{\prime}$ vs. $n$ considerando $h=10$ e $D=0,5$. . . . . . . . 28

3.2 Espaço de fases usando: (a) $\epsilon=10^{-3}$ e $\gamma=1 / 2$, (b) $\epsilon=10^{-2}$ e $\gamma=1 . \quad$. 29

3.3 (a) $D$ vs. $h$ para diferentes valores de $\epsilon$ e $\gamma$. (b) Depois de uma reescala nos eixos, as curvas colapsaram para pequenos valores de $h$. (c) Depois de uma reescala apropriada nos eixos, todas as curvas colapsaram para altos valores de $h . \ldots \ldots \ldots \ldots \ldots \ldots \ldots$

3.4 Para $\gamma=3 / 4$ temos: (a) Um gráfico do Histograma em função de $n_{p}$, onde destacamos como encontrar $n_{p}$; (b) $A$ vs. $h$, onde a inclinação é igual à $-1,97(1)$; (c) $n_{p}$ vs. $h$ para diferentes valores de $\epsilon: \epsilon=10^{-5}$, $\epsilon=3 \times 10^{-5}$ e $\epsilon=10^{-4}$ e (d) $n_{p}$ vs. $h / \epsilon \ldots \ldots \ldots \ldots \ldots$

4.1 Esquema ilustrativo do potencial $V(x, t)$ dado pela Eq. (4.1). . . . . . 36

4.2 (a) Uma cadeia contendo infinitos poços de potencial simétricos, onde os fundos destes se movem periodicamente e sincronizados no tempo; (b) Um único poço de potencial; (c) Poço de potencial apresentado em (b) mas que foi cortado ao meio por causa de sua simetria. 
4.3 Espaço de fase para o mapeamento (4.2). Os círculos correspondem aos pontos fixos elípticos ao passo que aqueles em formato de cruz são pontos fixos hiperbólicos. As cores vermelhas são usadas no primeiro tratamento (i) e as azuis no segundo tratamento (ii). Os parâmetros de controle usados são $r=1, N_{c}=33,18$ e $\delta=0,5 \ldots \ldots \ldots$

4.4 (a) Posição da primeira curva invariante do tipo spanning utilizando os parâmetros de controle $N_{c}=9, N_{c}=10$ e $N_{c}=20$. (b) Gráfico de $e_{\min } \times N_{c}$. Os parâmetros de controle utilizados foram $r=1$ e $\delta=0,5$.

4.5 (a) Gráfico do histograma do número de partículas que atingiram a altura $h$ em um certo número de iteradas $n$ para diferentes valores de $N_{c}$. (b) Colapso de diferentes histogramas em uma única curva, confirmando uma invariância de escala. Os outros parâmetros usados nas simulações foram: $r=1$ e $\delta=0,5 \ldots \ldots \ldots \ldots \ldots$

4.6 Gráfico de $n_{p} \times N_{c}$. Um ajuste em lei de potência fornece $\gamma=1,26(3)$.

4.7 (a) Gráfico de $P$ vs $n$ para diferentes posições de $h(h=4,6,10$ e 12). Os parâmetros de controle usados foram $N_{c}=33,18, r=1$ e $\delta=0,5$. As linhas pontilhadas são obtidas através de um ajuste exponencial. (b) Colapso, para pequenos valores de $n$, de todas as curvas mostradas em (a) depois de uma reescala $n \rightarrow n / h^{2,5}$.

4.8 Expoente do decaimento exponencial $\gamma$ como função do $h$ para três diferentes valores de $N_{c}: N_{c}=33,18, N_{c}=66,36$ e $N_{c}=99,54$. Os outros parâmetros de controle utilizados foram $r=1$ e $\delta=0,5$. As linhas retas são o melhor ajuste para cada $N_{c}$ escolhido. . . . . . . . . . .

4.9 Gráfico das funções: (a) $f_{1}(\phi)$; (b) $f_{2}(\phi)$; (c) $f_{3}(\phi)$; (d) $f_{4}(\phi)$ usando $q=2$. As linhas pontilhadas vermelhas correspondem a função $f_{0}(\phi)$ que foi usada como comparação. . . . . . . . . . . . . . .

4.10 Espaço de fases para o mapeamento (4.2) usando como parâmetros de controle $N_{c}=33,18, r=1$ e $\delta=0,5$ para: (a) $f_{0}(\phi)$, (b) $f_{1}(\phi)$, (c) $f_{2}(\phi)$ e $(\mathrm{d}) f_{3}(\phi)$. As curvas vermelhas representam a posição da primeira curva invariante spanning. . . . . . . . . . . . . . .

4.11 (a) Gráfico do expoente de Lyapunov como função de $n$ para cinco diferentes condições iniciais e considerando a função $f_{0}$. Os parâmetros usados foram $N_{c}=10, r=1$ e $\delta=0,5$. Para as funções $f_{0}, f_{1}, f_{2}$ e $f_{3}$ temos: (b) $\bar{\lambda}$ vs $N_{c}$ para valores fixos de $r=1$ e $\delta=0,5$; (c) $\bar{\lambda}$ vs $r$ para $N_{c}=33,18$ e $\delta=0,5$; (d) $\bar{\lambda}$ vs $\delta$ considerando $N_{c}=33,18$ e $r=1 . .$.

4.12 Gráfico de: (a) $\omega$ vs $n$ usando $f_{0}$ e considerando $N_{c}=100, r=1 \mathrm{e}$ $\delta=0,5$. Para as funções $f_{0}, f_{1}, f_{2}$ e $f_{3}$ temos: (b) $\omega_{\text {sat }}$ vs $N_{c}$ para valores fixos de $r=1$ e $\delta=0,5$; (c) $\omega_{\text {sat }}$ vs $r$ para $N_{c}=33,18$ e $\delta=0,5$ (d) $\omega_{\text {sat }}$ vs $\delta$ considerando $N_{c}=300$ e $r=1$. Valores numéricos para os expoentes são mostrados na Tabela $4.1 . \ldots \ldots \ldots \ldots$ 
4.13 Gráfico do crossover $n_{x}$ como função dos parâmetros de controle, considerando diferentes funções $f_{0}, f_{1}, f_{2}$ e $f_{3}$ para: (a) $n_{x}$ vs $N_{c}$ para valores fixos de $r=1$ e $\delta=0,5$; (b) $n_{x}$ vs $r$ para $N_{c}=33,18$ e $\delta=0,5$ (c) $n_{x}$ vs $\delta$ para $N_{c}=300$ e $r=1 \ldots \ldots \ldots \ldots \ldots$

4.14 (a) Gráfico da posição da curva invariante spanning usando a função $f_{0}$ e considerando $r=1$ e $\delta=0,5$ para $N_{c}=800, N_{c}=900$ e $N_{c}=1000$. (b) Gráfico de $e_{\min }$ vs $N_{c}$ com inclinação $\alpha_{1}$ para diferentes funções $f_{i}(i=0,1,2,3)$; (c) $e_{\min }$ vs $r$ com inclinação $\alpha_{2}$; (d) $e_{\min }$ vs $\delta$ com inclinação $\alpha_{3} \ldots \ldots \ldots \ldots \ldots \ldots \ldots \ldots \ldots$

4.15 Grade de condições iniciais $\left(\phi_{0}, e_{0}\right)$ mostrando o logaritmo do tempo de escape (LTE) para: (a) $r=80$; (b) $r=100$; (c) $r=200$. Número de diferentes tempos de retorno (NDTR) para: (d) $r=80$; (e) $r=100$; (f) $r=200$. As janelas de escape são situadas em $e_{1}<59$ e $e_{1}>75$. A função $f_{0}$ foi usada para obter os resultados. . . . . . . . . . .

4.16 Grade com diferentes condições iniciais $\left(\phi_{0}, e_{0}\right)$ mostrando como cores o logaritmo do tempo de escape (LTE) para: (a) $r=400$; (b) $r=600$; (c) $r=700$. Número de diferentes tempos de retorno (NDTR) para: (d) $r=400$; (e) $r=600$; (f) $r=700$. As janelas de escape são situadas em $e_{1}<117$ e $e_{1}>127$. A função $F_{0}$ foi usada para obter os resultados. . .

4.17 Gráfico de $e_{\min }$ vs $N_{c}$ para $q=2, q=25, q=50$ e $q=100$. Um ajuste em lei de potência nos fornece a inclinação 2/3; (b) Gráfico de $e_{\min }$ vs $q$ com inclinação $0,65557(7)$; (c) Colapso das curvas mostradas no item (a) em um único e universal gráfico depois da transformação $e_{\min } \rightarrow e_{\min } / a^{0,65557 \ldots \ldots \ldots \ldots \ldots \ldots \ldots}$

5.1 Esquema ilustrativo da fronteira e dos ângulos envolvidos na descrição do mapa. Nesta figura temos que $p=2$ e $\epsilon>\epsilon_{c}=0,2 \ldots \ldots$. . . . 58

5.2 Gráfico mostrando: (a) $\alpha \times \theta$ para $\Omega=0$; (b) $\alpha \times \theta$ para $\Omega=1$ e; (c) $\alpha \times(\theta+\Omega t)(\bmod 2 \pi)$. Os parâmetros usados foram $\epsilon=0,1$ e a condição inicial foi $V_{0}=3, \theta_{0}=\pi, t_{0}=0$ e $\alpha_{0}=\pi / 2-0,1$. A órbita foi evoluída até $2 \times 10^{5}$ colisões com a fronteira. . . . . . . . . . . .

5.3 Gráfico da coordenada angular do ponto fixo de período dois para diferentes valores de $V_{0}$ e usando $\epsilon=0,1$ e $\Omega=1$. A linha pontilhada é dada por $\alpha^{*}=\pi / 2 \ldots \ldots \ldots \ldots \ldots \ldots \ldots$

5.4 Gráfico de $V$ vs. $\theta^{\prime}$ para diferentes valores de $V_{0}$ e considerando $\epsilon=0,1$, $\Omega=1, \alpha_{0}=\pi / 2-0,1$ e $\theta_{0}=0,16$. Cada órbita foi iterada $10^{4}$ vezes.

5.5 Gráfico de uma órbita usando $V_{0}=3, \theta_{0}=\pi / 2$ e $t_{0}=0$ para: (a) $\Omega=0$ e $\alpha_{0}=\pi / 2$ (ponto fixo de período dois); (b) $\Omega=1$ e $\alpha_{0}=1,28312$ (outro exemplo de trajetória em um ponto fixo); (c) O mesmo mostrado no item (b) considerando a reescala $\theta \rightarrow \theta+\Omega t$; (d) Um exemplo de saltos curtos ao longo da fronteira para $\Omega=1$ e $\alpha_{0}=2,9$ (correspondendo a uma órbita rotator $) \ldots \ldots \ldots \ldots \ldots \ldots \ldots$ 
5.6 (a) Gráfico de $R_{L}$ em função de $V_{0}$. Depois de um ajuste em lei de potência o expoente obtido foi $0,996(1)$; (b) $R_{L}$ vs. $\Omega$ e o expoente obtido foi $-0,995(2) \ldots \ldots \ldots \ldots \ldots \ldots$

5.7 Diagrama de $\alpha \times \theta$ onde as cores representam as mínimas (a,c) e máximas $(\mathrm{b}, \mathrm{d})$ velocidades ao longo da órbita. O tempo inicial foi $t_{0}=\mathrm{e}$ : $(\mathrm{a}, \mathrm{c})$

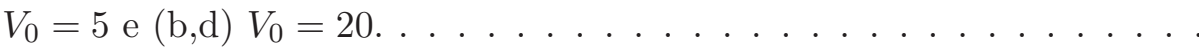

5.8 Gráfico de: (a) $\alpha \times \theta$ para $\Omega=0$ e; (b) $\alpha \times \theta^{\prime}$ para $\Omega=1$. Os parâmetros usados foram $\epsilon=0,3$ para a condição inicial $\alpha_{0}=\pi / 2-0,1, \theta_{0}=\pi$, $t_{0}=0$ e $V_{0}=3 \ldots \ldots \ldots \ldots \ldots \ldots \ldots \ldots \ldots \ldots \ldots \ldots \ldots \ldots \ldots$

5.9 Gráfico de $\bar{V}$ em função de $n$ usando 300 diferentes condições iniciais escolhidas aleatoriamente $\left(t_{0} \in(0,2 \pi], \theta_{0} \in(0,2 \pi]\right.$ e $\left.\alpha_{0} \in(0, \pi]\right)$ para cada $V_{0}$. As órbitas foram iteradas $10^{8}$ vezes. . . . . . . . . . 64

5.10 (a) Gráfico de $n_{x} v s$. $V_{0}$. Um ajuste em lei de potência fornece o expoente $z=4,96(4)$; (b) Gráfico da velocidade inicial do plato $V_{i p}$ em função de $V_{0}$. A inclinação encontrado após um ajuste em lei potência foi $\alpha=$

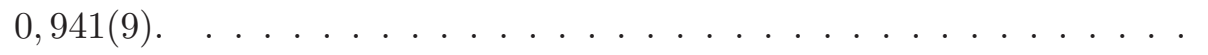

5.11 Reescala de todas as curvas mostradas na figura 5.9 em uma única e universal curva, onde as transformações nos eixos usadas foram $(V \rightarrow$ $\left.V / V_{0}^{0,941}\right)$ e $\left(n \rightarrow n / V_{0}^{4,96}\right) . \ldots \ldots \ldots \ldots$ 
LISTA DE FIGURAS 


\section{Lista de Tabelas}

4.1 Expoentes obtidos analisando o desvio da energia média. . . . . . . . . . 49

4.2 Expoentes obtidos pela mínima energia ao longo da curva invariante

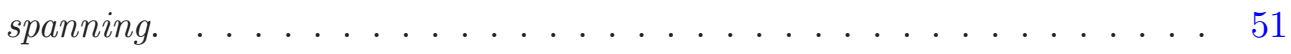


LISTA DE TABELAS 


\section{Bibliografia}

[1] J. P. Eckmann e D. Ruelle, Rev. Mod. Phys 57, 617 (1985). 1

[2] N. Chernov, R. Markarian, Chaotic Billiards, American Mathematical Society, Vol. 127 (2006). 1, 2

[3] Lichtenberg A J and Lieberman M A 1992 Regular and Chaotic Dynamics, Applied Mathematical Sciences Vol 38 (Springer-Verlag, New York). 1, 3, 18, 19, 26, 30

[4] R M May, Science, 86 645-647 (1974). 1

[5] N. Fiedler-Ferrara, C. P. C. do Prado, Caos Uma Introdução, Edgard Blucher (1994). 1, 41

[6] D. F. M. de Oliveira e E. D. Leonel, New J. Phys. 13, 123012 (2011). 1

[7] G. D. Birkhoff Dynamical Systems (Providence, RI: American Mathematical Society). 2

[8] Dullin H R 1998 Nonlinearity 11 151-173. 2

[9] E. D. Leonel, J. K. L. Silva, S. O. Kamphorst, Phys. A, 331, 435-447 (2004). 2

[10] E. D. Leonel, P. V. E. McClintock, J. K. L. Silva, Phys. Rev. Lett. 93, 014101 (2004). 2

[11] D. R. da Costa, A. L. P. Livorati, E. D. Leonel, Int. J. of Bifurcation and Chaos 22, 1250250 (2012). 2, 69

[12] J. Nagler, M. Krieger, M. Linke, J. Schönke, J. Wiersig, Phys. Rev. E, 75, 046204 (2007). 2

[13] C. P. Dettmann, O. Georgiou, Phys. D 238, 2395-2403 (2009). 2, 26, 44, 45

[14] C. P. Dettmann, O. Georgiou, arXiv:1006.1316v2 (2010). 2

[15] L. A. Bunimovich, C. P. Dettmann, Phys. Rev. Lett. 94100201 (2005). 2 
[16] C. P. Dettmann, E. D. Leonel, Phys. D 241, 403-408 (2012). 2, 32

[17] C. P. Dettmann, arXiv:1007.4166v1 (2010). 2

[18] E. Fermi, Phys. Rev. 75, 1169 (1949). 2

[19] S. E. Sklarz, D. J. Tannor, N. Khaneja, Phys. Rev. A 69, 053408 (2004). 2

[20] K. Nakamura e T. Harayama, Quantum Chaos and Quantum Dots, Oxford University Press, Oxford (2004). 2

[21] M. Steiner, M. Freitag, V. Perebeinos, J. C. Tsang, J. P. Small, M. Kinoshita, D. Yuan, J. Liu, P. Avouris, Nature Nanotech. 4, 320 (2009). 2

[22] A. K. Karlis, P. K. Papachristou, F. K. Diakonos, V. Constantoudis, and P. Schmelcher, Phys. Rev. Lett. 97, 194102 (2006). 2

[23] A. C. J. Luo, ASME J. Vib. Acoust. 124, 420 (2002). 2

[24] A. C. J. Luo, R. P. S. Han, Nonlinear Dynam. 10, 1 (1996). 2

[25] F. Lenz, C. Petri, F. K. Diakonos, and P. Schmelcher, Phys. Rev. E 82, 016206 (2010). 3

[26] L. D. Pustyl'nikov, Theor. Math. Phys. 57, 1035 (1983). 3

[27] A. L. P. Livorati, T. Kroetz, C. P. Dettmann, I. L. Caldas and E. D. Leonel, Phys. Rev. E 86, 036203 (2012). 3

[28] M. R. Silva; D. R. da Costa e E. D. Leonel, J. Phys. A: Math. and Theor. 45, 265101 (2012). 3, 7, 8, 10, 23, 67, 69

[29] E. D. Leonel, D. R. da Costa e C. P. Dettmann, Phys. Lett. A 376, 421-425 (2012). $3,7,8,20,23,67,69$

[30] D. R. da Costa, M. R. Silva e E. D. Leonel, Commun. Nonlinear. Sci. Numer. Simul. 19, 842-850 (2014). 3, 7, 8, 13, 23, 67, 69

[31] J. A. de Oliveira, C. P. Dettmann, D. R. da Costa, E. D. Leonel, Phys. Rev. E 87, 062904 (2013). 4, 25, 33, 68, 69

[32] D. R. da Costa, C. P. Dettmann e E. D. Leonel, Phys. Rev. E 83, 066211 (2011). $4,26,36,41,45,68,69$

[33] D. R. da Costa, M. R. Silva, J. A. de Oliveira e E. D. Leonel, Phys. A 391, 3607-3615 (2012). 4, 36, 39, 42, 45, 68, 69

[34] D. R. da Costa, I. L. Caldas e E. D. Leonel, Phys. Lett. A 377, 1814 (2013). 4, $36,38,55,68,69$ 
[35] D. R. da Costa, D. F. M. Oliveira, E. D. Leonel, Commun. Nonlinear. Sci. Numer. Simul. 19, 1926-1934 (2014). 4, 57, 66, 68, 69

[36] D. Le Sage et. al, Phys. Rev. B 85, 121202(R) (2012). 8

[37] A. G. York et. al, Phys. Rev. Lett. 100195001 (2008). 8

[38] J. P. Palastro et. al, Phys. Rev. E 77, 036405 (2008). 8

[39] L. P. Kouwenhoven et. al, Phys. Rev. Lett. 65, 361 (1990). 8, 36

[40] A. L. Virovlyansky E G. M. Zaslavsky, Chaos 10, 211 (2000). 8

[41] I. P. Smirnov, A. L. Virovlyansky e G. M. Zaslavsky, Phys. Rev. E 64, 036221 (2001). 8

[42] A. Iomin e Yu Bliokh, Commun. Nonlinear. Sci. Numer. Simul. 8, 389 (2003). 8

[43] E. Hecht, Optics, 4th ed., Reading, MA: Addison-Wesley (2002). 13

[44] A. Lakhtakia, Optik 90, 184 (1992). 14

[45] D. B. Brewster, Philos. Trans. R. Soc. Lond. 105, 105 (1815). 14

[46] A. Lakhtakia, OSA Optics News 15, 14 (1989). 14

[47] http://refractiveindex.info. Acessado em 15 de Abril de 2012. 16, 18, 72

[48] A. F. Rabelo e E. D. Leonel. Brazilian J. Phys. 38, 54 (2008). 21, 22

[49] Zaslavsky G. M., Sagdeev R. D., Usikov D. A. and Chernikov A. A, Weak Chaos and Quasi-Regular Patterns. Cambridge University Press, Cambridge, (1991). 26

[50] G. M. Zaslavsky, Hamiltonian chaos and fractional dynamics. Oxford University Press, Oxford, (2005). 26

[51] I. I. Shevchencko, New Astron. 16, 94(2011). 26

[52] M. A. Lieberman, A. J. Lichtenberg, Phys. Rev. A 5, 1852 (1971). 26

[53] G. A. Luna-Acosta, J. A. Mendez-Bermudez, and F. M. Izrailev, Phys. Rev. E 64, 036206 (2001); Phys. Lett. A 274, 192 (2000). 26

[54] G. A. Luna-Acosta, G. Orellana-Rivadeneyra, A. Mendoza-Galván, and C. Jung, Chaos, Solitons and Fractals, 12, 349 (2001). 26, 47, 51

[55] J. L. Mateos, Phys. Lett. A, 256, 113 (1999). 26

[56] L. D. Pustylnikov, Trans. Moscow Math. Soc. 2, 1 (1978). 26

[57] L. A. Bunimovich and C. P. Dettmann, Europhys. Lett., 80, 40001 (2007). 26 
[58] E. G. Altmann, S. E. Portela and Tamas Tel, arxiv:1208.0254. 26

[59] P. Gaspard and G. Nicolis, Phys. Rev. Lett. 65, 1693(1990). 27

[60] J. R. Dorfman and P. Gaspard, Phys. Rev. E 51, 28(1995). 27

[61] W. J. Adams, The Life and times of the Central Limit Theorem. 2.ed. American Mathematical Society, Providence (2009). 27

[62] H. Fischer, History of the Central Limit Theorem. Springer, New York (2011). 27

[63] I. Eliazar, J. Klafter, Chem. Phys. 370, 290 (2010). 27

[64] H. J. Hilhorst, Braz. J. Phys 39, 371 (2009). 27

[65] S. Umarov, C. Tsallis, S. Steinberg, Milan J. Math. 76, 307 (2008). 27

[66] G. M. Zaslavsky, Phys. Rep. 371, 461 (2002). 27

[67] E. D. Leonel, J. A. de Oliveira and F. Saif. J. Phys. A 44, 302001 (2011). 30, 51

[68] C. F. F. Karney, Phys. D 8, 360 (1983). 32

[69] M. F. Demers and L.-S. Young, Nonlinearity 19, 377 (2006). 32

[70] V. A. Avetisov and S. K. Nechaev, Phys. Rev. E 81, 046211 (2010). 32

[71] D. L. Shepelyansky, Phys. Rev. E 82, 055202(R) (2010). 32

[72] G. Cristadoro, R. Ketzmerick, Phys. Rev. Lett. 100, 184101 (2008). 32

[73] S. S. Abdullaev and K. H. Spatschek, Phys. Rev. E 60, R6287 (1999). 32

[74] H. Henry et. al., Chaos 9, 381 (1999). 32

[75] N. Buric, A. Rampioni, G. Turchetti and S. Vaienti, J. Phys. A: Math. Gen. 36, L209 (2003). 32

[76] M. Buttiker, R. Landauer, Phys. Rev. Lett. 49, 1739 (1982). 35

[77] M. Leng, C. S. Lent, Phys. Rev. Lett. 71, 137 (1993). 36

[78] L. P. Kouwenhoven, S. Jauhar, J. Orenstein, P. L. McEuen, Phys. Rev. Lett. 73, 3443 (1994). 36

[79] R. B. Hwang, IEEE T. Antenn. Propag., 54, 755 (2006). 36

[80] E. D. Leonel, P. V. E. McClintock, Phys. Rev. E 70, 016214 (2004). 36

[81] P. K. Papachristou, F. K. Diakonos, E. Mavrommatis, V. Constantoudis, Phys. Rev. E 64, 016205 (2001). 36 
[82] P. K. Papachristou, F. K. Diakonos, V. Constantoudis, P. Schmelcher, L. Benet, Phys. Lett. A 306, 116 (2002); Phys. Rev. E 70, 056215 (2004). 36

[83] J. Wu, et al., Phys. Lett. A 262, 245 (1999). 36

[84] E. G. Altmann, T. Tél, Phys. Rev. E 79, 016204 (2009). 45

[85] J. Andreasen, H. Cao, J. Wiersig e A. E. Motter, Phys. Rev. Lett. 103, 154101 (2009). 57

[86] A. Loskutov, A. B. Ryabov, L. G. Akinshin, J. Phys. A 33, 7973 (2000). 57

[87] D. F. M. Oliveira, E. D. Leonel, Phys. A 389, 1009 (2010). 57

[88] A. Loskutov, A. Ryabov, J. Stat. Phys. 108, 995 (2002). 57

[89] A. K. Karlis, P. K. Papachristou, F. K. Diakonos, V. Constantoudis, P. Schmelcher, Phys. Rev. E 76, 016214 (2007). 57

[90] F. Lenz, F. K. Diakonos, P. Schmelcher, Phys. Rev. Lett. 100, 014103 (2008). 57, 58

[91] D. F. M. Oliveira, M. Robnik, Phys. Rev. E 83, 026202 (2011). 57

[92] E. D. Leonel; A. L. Bunimovich, Phys. Rev. Lett. 104, 224101 (2010). 57, 58

[93] D. F. M. Oliveira, E. D. Leonel, Commun. Nonlinear. Sci. Numer. Simul. 15, 1092 (2010). 59, 60

[94] M. Robnik e M. V. Berry, J. Phys. A: Math. Gen. 18, 1361-1378 (1985). 62, 63

[95] M. V. Berry e E. C. Sinclair, J. Phys. A: Math. Gen. 30, 2853-2861 (1997). 62

[96] E. D. Leonel, D. F. M. Oliveira, A. Loskutov, Chaos 19, 033142 (2009). 65

[97] B. Batistić e M. Robnik, J. Phys. A: Math. Theor. 44, 365101 ( 2011). 65

[98] D. R. da Costa, I. L. Caldas e E. D. Leonel, submetido para publicação na Appl. Math. Comput. (2013). 69

[99] D. R. da Costa, C. P. Dettmann e E. D. Leonel, submetido para publicação na Commun. Nonlinear. Sci. Numer. Simul. (2013). 69

[100] M. Hansen, D. R. da Costa, D. F. M. Oliveira e E. D. Leonel, submetido para publicação na Appl. Math. Comput.. 69

[101] D. R. da Costa, C. P. Dettmann e E. D. Leonel, submetido para publicação na Phys. Rev. E (2013). 69 
BIBLIOGRAFIA 